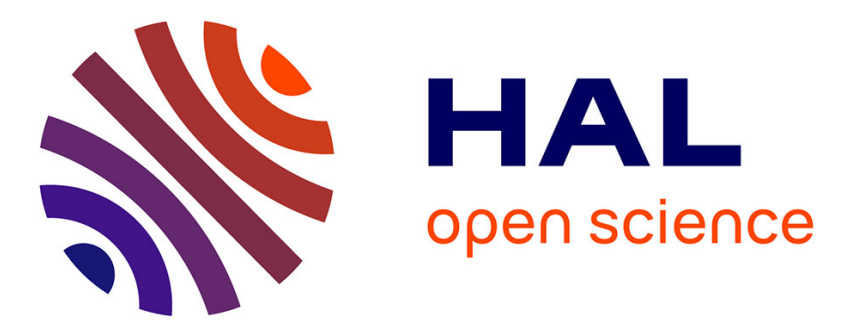

\title{
Évaluation des dommages myocardiques en post opératoire de chirurgie vasculaire et thoracique par imagerie cardiaque par résonance magnétique \\ Eugénie Tith
}

\section{- To cite this version:}

Eugénie Tith. Évaluation des dommages myocardiques en post opératoire de chirurgie vasculaire et thoracique par imagerie cardiaque par résonance magnétique. Médecine humaine et pathologie. 2012. dumas-00746005

\section{HAL Id: dumas-00746005 https://dumas.ccsd.cnrs.fr/dumas-00746005}

Submitted on 26 Oct 2012

HAL is a multi-disciplinary open access archive for the deposit and dissemination of scientific research documents, whether they are published or not. The documents may come from teaching and research institutions in France or abroad, or from public or private research centers.
L'archive ouverte pluridisciplinaire HAL, est destinée au dépôt et à la diffusion de documents scientifiques de niveau recherche, publiés ou non, émanant des établissements d'enseignement et de recherche français ou étrangers, des laboratoires publics ou privés. 


\title{
FACULTE MIXTE DE MEDECINE ET DE PHARMACIE DE ROUEN
}

\author{
THESE POUR \\ LE DIPLOME D'ETAT DE DOCTEUR EN MEDECINE \\ ANESTHESIE - REANIMATION
}

Présentée et soutenue publiquement le 18 octobre 2012

Par

Melle Eugénie TITH, née le 6 février 1982 à Paris

\section{EVALUATION DES DOMMAGES MYOCARDIQUES EN POST OPERATOIRE DE CHIRURGIE VASCULAIRE ET THORACIQUE PAR IMAGERIE CARDIAQUE PAR RESONANCE MAGNETIQUE}

Président du jury : $\quad$ M. Pr Bertrand DUREUIL

Directrice de thèse : Mme le Dr Marie-Mélody DUSSEAUX 


\section{A Monsieur le Professeur Bertrand Dureuil, \\ Pour l'honneur que vous me faites de présider ce jury de thèse et pour votre présence bienveillante tout au long de mes études, \\ Puissiez-vous trouver ici le témoignage de mon admiration et de ma gratitude.}

\section{A Monsieur le Professeur Didier Plissonnier,}

Pour l'honneur que vous me faites de siéger parmi mes juges,

Je n'oublierai jamais la magie de la première chirurgie aortique à la laquelle j'ai assisté.

Veuillez trouver ici le témoignage de mon respect et de ma gratitude.

\section{A Monsieur le Professeur Jean Nicolas Dacher,}

Pour l'honneur que vous me faites de juger ce travail.

Merci de votre gentillesse et de votre disponibilité.

Veuillez trouver ici la marque de mon plus profond respect et de ma gratitude.

\section{A Monsieur le Docteur Matthieu Godin,}

Merci de me faire l'honneur de juger cette thèse.

Veuillez trouver dans ce travail la marque de mon respect.

\section{A Madame le Docteur Marie Mélody Dusseaux,}

Pour l'honneur que tu m'as fait en acceptant de diriger ce travail, Merci de ta patience et de ta disponibilité.

\section{A Madame le Docteur Sylvie Godier,}

Pour ta gentillesse et ta patience dont tu as fait preuve, Merci pour ton soutien et ta présence à toutes les étapes de cette thèse. 


\section{A tous mes maîtres d'internat,}

Merci de votre patience, votre soutien et de tous vos enseignements durant ces 5 années. Tout particulièrement :

A Véronique et Maamar, beaucoup imités mais jamais égalés, ne changez rien,

A Alexandra et Ygal, le mari d'Alexandra, merci pour vos précieux conseils et félicitations pour vos deux merveilles,

A Dominique et Christophe, votre patience est sans limite,

A Gaëlle Pfister, tu as su trouver les mots dans la difficulté,

\section{A tous les internes croisés, anesthésistes et non anesthésistes,}

Merci de votre accueil et votre présence enrichissante.

Alice, Marie Laure, Elodie et Fabien, merci de votre soutien.

Ophélie, merci pour les hémato party!

\section{Aux équipes du bloc opératoire et de la réanimation du Havre,}

J'ai fait mes armes avec vous avant de rejoindre Rouen,

Je n'oublierai jamais mon premier jour au bloc opératoire et l'accueil plus que chaleureux.

J'ai (enfin) eu mon permis de conduire.

\section{A l'équipe de réanimation d'Elbeuf,}

Je vous retrouve bientôt mais du côté du bloc opératoire,

Et pour quelques nuits par mois en réanimation.

\section{Aux équipes du centre hospitalier du Belvédère,}

J'espère bientôt atteindre le niveau 2 en connaissance du col.

A Samuel pour sa bienveillance, sa disponibilité et son humour.

A Anne Sophie, bienvenue dans la région. Merci pour tes conseils et ton soutien dans cette dernière ligne droite.

A Nabil, pour les cours d'accouchement à l'algérienne et les cours de vocabulaire. 


\section{To my beloved family,}

Hong Kong, Ottawa, Toronto, Beijing, Phnom Penh...

Many thanks to all of you for your support and help.

Even though you're not here today, I'm thinking of you.

\section{A ma belle-famille,}

Vous m'avez accueilli à bras ouverts,

Yveline, Jean Gabriel, Mélaine, Antoine, Margaux, votre belle-fille, belle-sœur, tata Ninie, profite de cette occasion pour vous remercier.

\section{A mes amis de Paris,}

Merci de votre présence et de votre soutien indéfectible depuis l'école primaire, le collège, le lycée, la $1^{\text {ère }}$ année de médecine jusqu'à aujourd'hui : Audrey, Isabelle, Noémie, Caroline, Rodolfo, Léa, Aurélie, Sébastien, Perrine, Luka, Nathalie, Mireille, Mathilde, Xavier, Elsa, Daphné, Aurélie, Sophie, Julien, Marie Laure, Fabrice,...

\section{A mes amis de Normandie,}

Merci de vos encouragements dignes d'un tour de France pour cette dernière ligne droite : Audrey, Franck, Valéry, Fabrice, Sylvie, Maxime, Loïc, Stania, Clara, Quentin, Cynthia, Fred, Angeline, Antoine, Nolwenn, Tiphaine, Laetitia, Jérôme, Jérémie, Alexis, Christian, Aurélie, Ambre, Loïc...

\section{A Gregory,}

3 mots pour résumer toute mon affection : merci pour tout. 
TABLE DES MATIERES 
I. LES DOMMAGES MYOCARDIQUES PERI OPERATOIRES EN CHIRURGIE NON CARDIAQUE. 14

A. Définition de l'infarctus du myocarde ............................................................

B. Physiopathologie des dommages myocardiques post opératoires.......................15

C. Incidence des dommages myocardiques post opératoires …............................18

D. Retentissement pronostique des dommages myocardiques post opératoires ........19

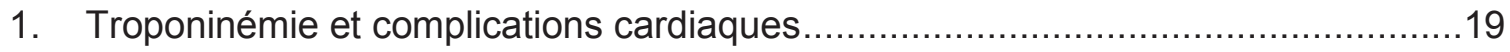

2. Troponinémie et mortalité post opératoire ......................................................19

\section{GESTION PERI OPERATOIRE DES DOMMAGES MYOCARDIQUES POST}

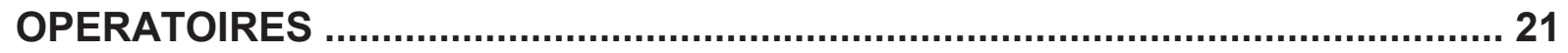

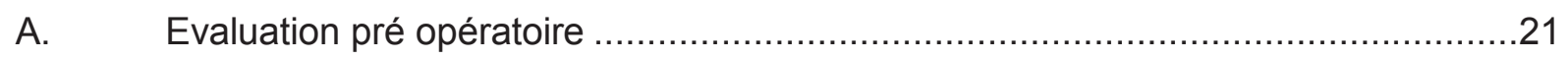

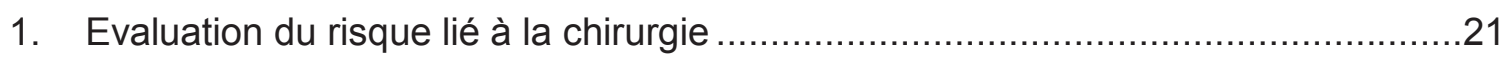

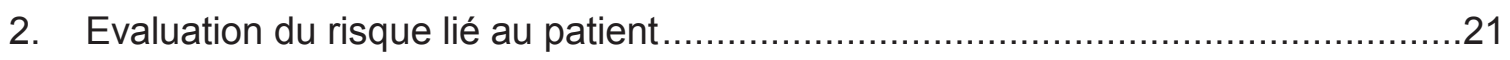

3. Score de Lee et Revised Cardiac Risk Index (RCRI) ....................................23

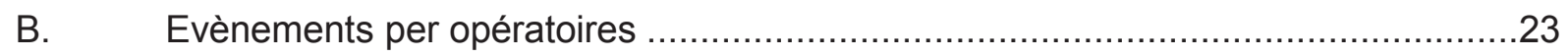

C. Diagnostic des dommages myocardiques post opératoires ...............................24

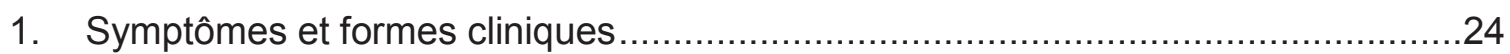

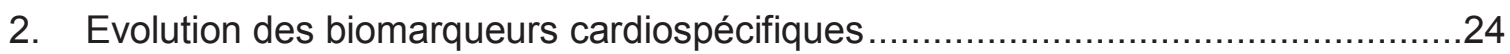

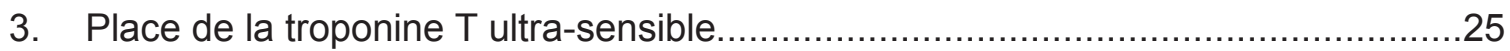

D. Prise en charge des dommages myocardiques en post opératoire :

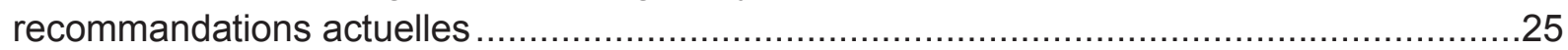

III. PLACE DE L'IMAGERIE DANS L'EVALUATION DU DMPO ........................ 27

A. Territoires d'ischémie myocardique et importance de la micro-vascularisation .......27

B. La coronarographie, gold standard dans l'exploration des cardiopathies

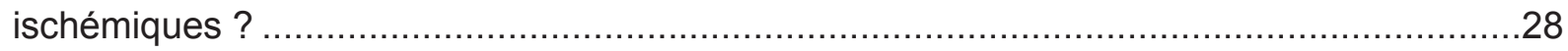

C. Explorations fonctionnelles non invasives disponibles à l'heure actuelle en post

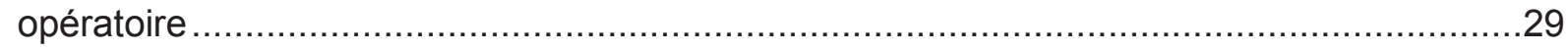

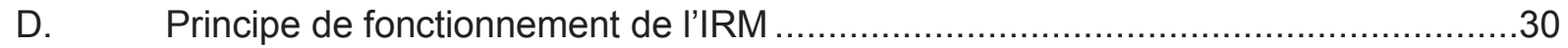

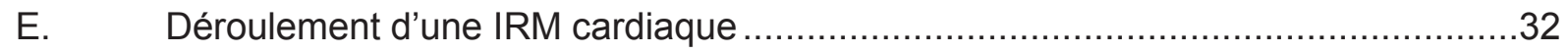

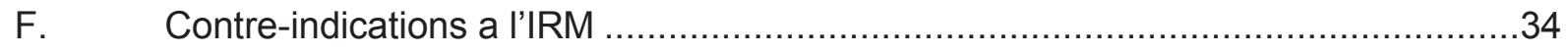

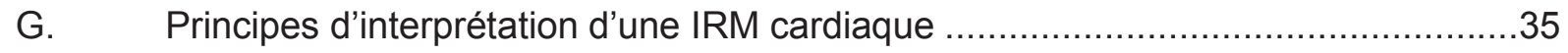

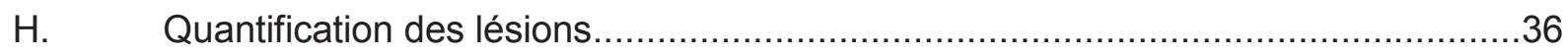


I. CADRE DE L'ETUDE

A. Type d'étude ……………

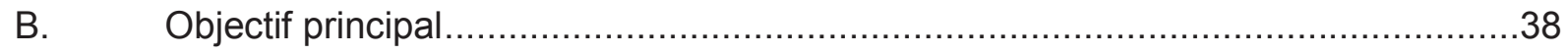

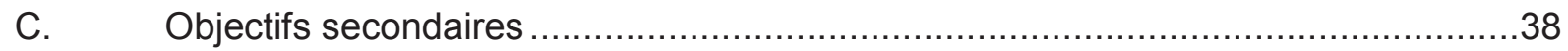

II. CRITERES D'INCLUSION ET D'EXCLUSION............................................. 38

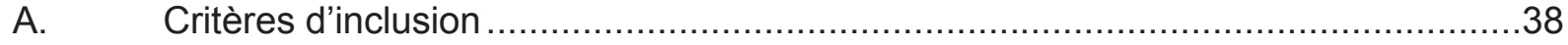

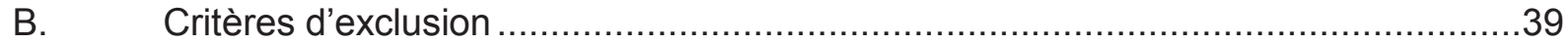

III. DEROULEMENT DE L'ETUDE

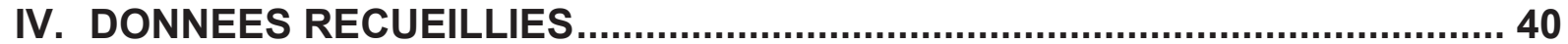

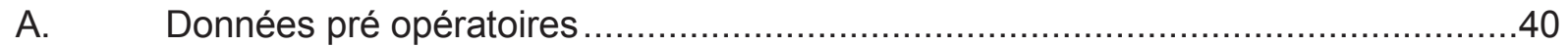

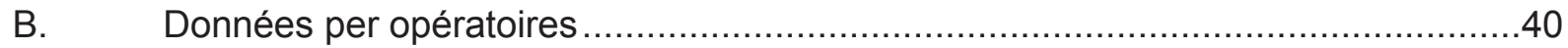

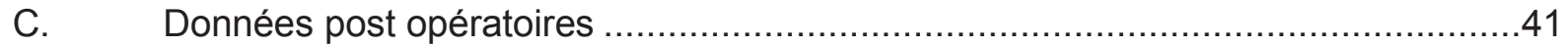

I. DESCRIPTION DE LA POPULATION ..................................................... 43

A. Patients ayant présenté une élévation de troponine ...........................................43

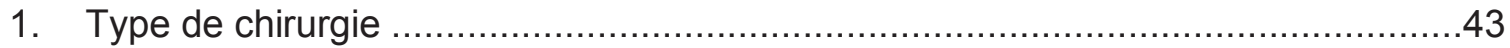

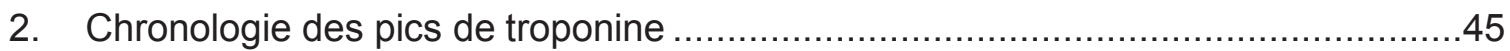

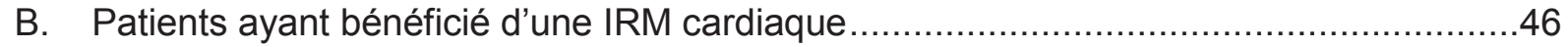

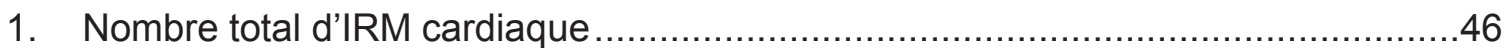

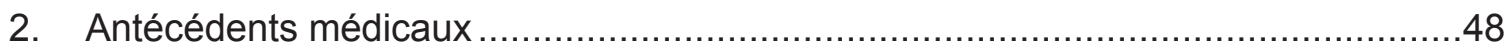

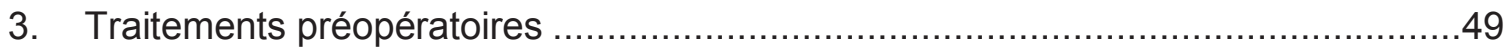

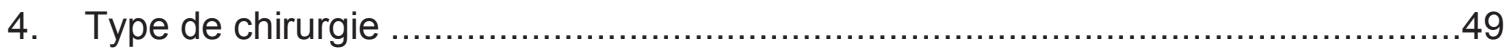

5. Evènements per et post opératoires immédiats ...............................................51

II. CINETIQUES DE TROPONINE CHEZ LES 24 PATIENTS ETUDIES .............. 52

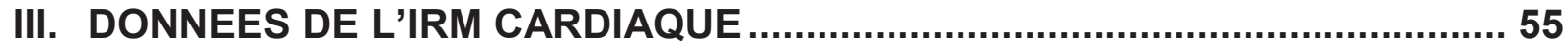

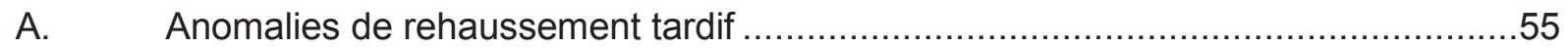

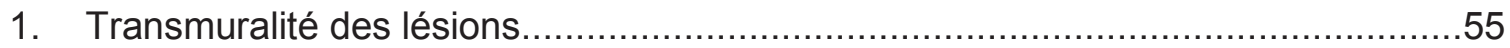

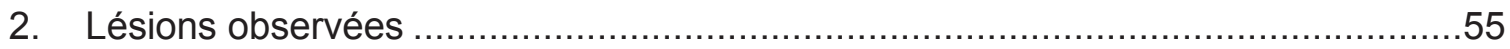

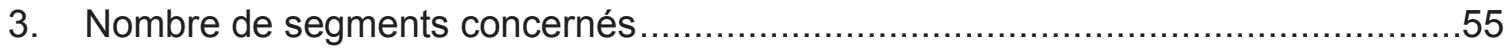

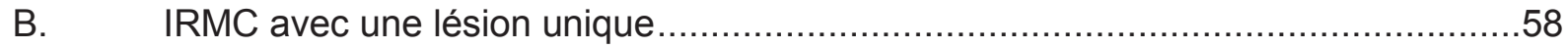

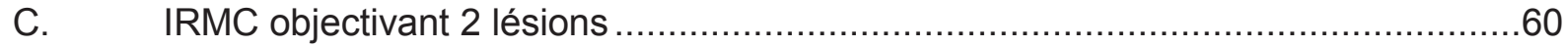




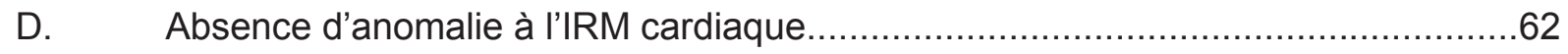

IV. EVOLUTION DES PATIENTS DU GROUPE ETUDIE ................................. 64

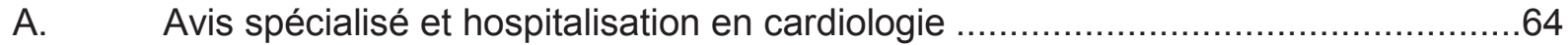

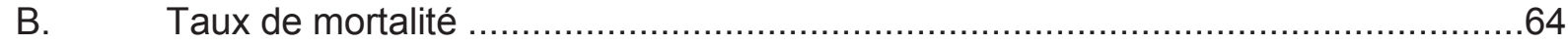

I. CONCERNANT LA POPULATION

A. Faible nombre de patients ayant bénéficié d'une IRMC par rapport au nombre de patients éligibles

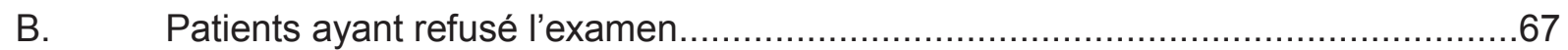

C. Perdus de vue et difficultés organisationnelles ...............................................67

II. CONCERNANT LE DOSAGE DE TROPONINE .......................................... 68

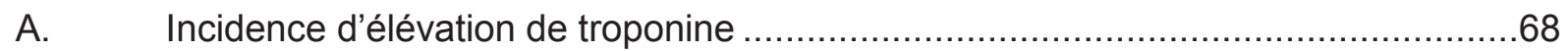

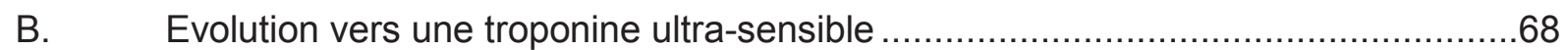

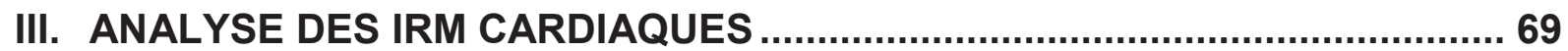

A. Localisation des dommages myocardiques post opératoires .............................69

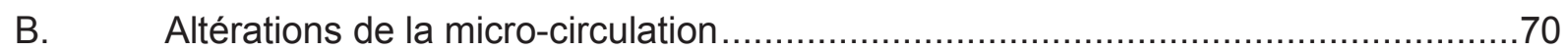

C. Comparaison des données de l'IRMC aux données cardiologiques pré et post

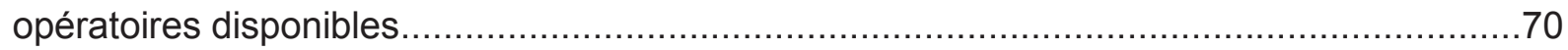

D. Anomalies à l'IRM cardiaque .................................................................. 71

IV. PRONOSTIC A 1 AN DES DOMMAGES MYOCARDIQUES POST

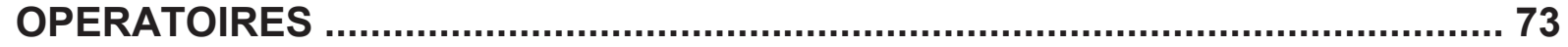

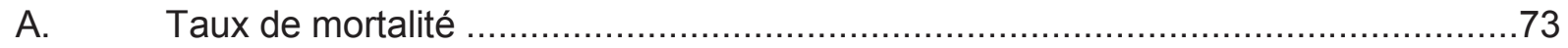

B. $\quad$ Prise en charge post opératoire immédiate …................................................

REFERENCES BIBLIOGRAPHIQUES 


\section{LISTE DES ABBREVIATIONS}

- IDM : infarctus du myocarde

- IMPO : infarctus du myocarde post opératoire

- DMPO : dommage myocardique post opératoire

- IRM : imagerie par résonance magnétique

- IRMC : imagerie par résonance magnétique cardiaque

- SCA : syndrome coronarien aigu

- ECG : électrocardiogramme

- IMC : indice de masse corporelle

- MET : équivalent métabolique

- HTA : hypertension artérielle

- Tnlc : troponine Ic

- TnTus : troponine T ultra-sensible

- RCRI : revised cardiac risk index

- ETT : échographie trans thoracique

- FMT : fréquence maximale théorique

- IVA : artère interventriculaire antérieure

- Cx : artère coronaire circonflexe

- VD : ventricule droit

- CD : coronaire droite

- IVP : artère interventriculaire postérieure

- FEVG : fraction d'éjection du ventricule gauche

- SCA : syndrome coronarien aigu

\section{RECAPITULATIF DES TABLEAUX}

Tableau I : caractéristiques démographiques et pré opératoires des 24 patients Tableau II : synthèse des 15 patients ayant présenté une anomalie à I'IRMC Tableau III : synthèse des 9 patients n'ayant pas présenté d'anomalie à I'IRMC 
Figure 1 : cinétiques de troponine lc dans les 3 types de DMPO : 1) DMPO précoce, 2) DMPO retardé, faisant suite à des nécroses myocardiques à minima mais répétées, 3) DMPO à minima sans évolution vers l'infarctus massif d'après Coriat et al. 2005

Figure 2 : profils de troponine lc en fonction du temps depuis l'admission en soins intensifs jusqu'à un DMPO. A) délai jusqu'à la détection de la $1^{\text {ère }}$ valeur anormale de troponine Ic, B) délai jusqu'à l'IMPO (troponine lc $\geq 1,5 \mathrm{ng} / \mathrm{ml}$ ), C) délai jusqu'au pic de troponine Ic d'après Le Manach et al. 2005

Figure 3: survie après un DMPO en fonction de la valeur de troponinémie post opératoire. Même les augmentations minimes de troponine prédisent une augmentation de la mortalité à long terme d'après Landesberg et al. 2003

Figure 4 : algorithme général de prise en charge du coronarien avant chirurgie non cardiaque d'après les recommandations formalisées d'expert SFAR/SFC 2010

Figure 5 : algorithme de prise en charge actuel des élévations de troponine en post opératoire d'après Piriou

Figure 6: "cascade ischémique " représentant les évènements faisant suite à une ischémie, d'après Yan et al. 2006

Figure 7 : émission du signal par la relaxation du spin d'hydrogène

Figure 8 : déroulement d'une IRMC

Figure 9 : évolution du gadolinium dans la paroi myocardique après injection d'après Germain et al.

Figure 10 : représentation des 17 segments cardiaques utilisés en tomographie cardiaque, en scintigraphie et en IRM cardiaque d'après Cerqueira et al. 2002

Figure 11 : territoires coronariens myocardiques d'après echorea.org

Figure 12 : répartition des indications chirurgicales vasculaires

Figure 13 : répartition des indications chirurgicales thoraciques

Figure 14: nombre de patients ayant présenté une élévation de troponine en fonction du jour post opératoire

Figure 15 : schéma d'inclusion de la population

Figure 16 : nombre de patients selon le RCRI

Figure 17 : nombre de patients ayant présenté un pic de troponine selon le jour post opératoire

Figure 18 : cinétique de troponine Ic des 13 patients ayant eu une IRMC 
Figure 19: cinétique de troponine $\mathrm{T}$ ultra-sensible des 11 patients ayant eu une IRMC

Figure 20 : histogramme représentant le nombre de patients ayant une troponinémie positive par jour post opératoire

Figure 21 : DMPO inféro-basal transmural avec un anévrysme en regard

Figure 22 : visualisation de 2 DMPO : un touchant la partie inférieure du ventricule droit et un la partie inféro-latérale du ventricule gauche

Figure 23: comparaison des images d'infarctus du myocarde canin en tomoscintigraphie myocardique (SPECT), d'IRM cardiaque (CMR) et de coupes histologiques, d'après Wagner et al. 2003

\section{ANNEXES}

\section{Annexe 1 : Revised Cardiac Risk Index (RCRI) et capacité fonctionnelle}

- Figure 24 : Revised Cardiac Risk Index

- Figure 25 : capacité fonctionnelle

\section{Annexe 2 : images de rehaussement tardif de lésions myocardiques}

- Figure 26 : infarctus myocardique antérieur aigu vu en IRM cardiaque sur les séquences de rehaussement tardif

- Figure 27: infarctus myocardique antéro-septal transmural vu en IRM cardiaque sur les séquences de rehaussement tardif

Annexe 3: exemple de protocole de prise en charge des syndromes coronariens aigus (SCA) mis en place aux hospices civils de Lyon

- Figure 28 : exemple de prise en charge de SCA post opératoire mis en place aux hospices civils de Lyon : définition du risque d'après Piriou et al. 2010

- Figure 29 : exemple de prise en charge de SCA post opératoire mis en place aux hospices civils de Lyon : orientation selon le risque d'après Piriou et al. 2010

- Figure 30 : exemple de prise en charge de SCA post opératoire mis en place aux hospices civils de Lyon : gestion du patient en cardiologie d'après Piriou et al. 2010 
INTRODUCTION 


\section{PREAMBULE}

En 1990, l'étude de Mangano constatait déjà que les complications cardiovasculaires étaient la principale cause de morbi-mortalité post opératoire en chirurgie non cardiaque ${ }^{1}$.

La connaissance de la physiopathologie de ces lésions myocardiques péri opératoires est meilleure au fil des années et a fait évoluer la définition de l'infarctus du myocarde, permettant d'identifier différents types d'infarctus du myocarde post opératoires (IMPO).

Encore aujourd'hui, la fréquence de l'élévation de troponine en post opératoire de chirurgie vasculaire et thoracique nous a incité à tenter de caractériser les dommages myocardiques en terme d'imagerie, dans le but, non pas d'en généraliser la pratique, mais d'authentifier les lésions myocardiques correspondantes. 


\section{LES DOMMAGES MYOCARDIQUES PERI OPERATOIRES EN CHIRURGIE NON CARDIAQUE}

\section{A. Définition de l'infarctus du myocarde}

La définition des dommages myocardiques a évolué au cours du temps notamment depuis l'apparition des marqueurs biologiques cardiospécifiques tels que les troponines. En 2007, un groupe de travail international, regroupant l'European Society of Cardiology (ESC), I'American College of Cardiology (ACC), I'American Heart Association (AHA) et la World Heart Federation (WHF), a standardisé une définition de l'infarctus du myocarde ${ }^{2}$.

L'infarctus aigu est alors défini par une augmentation ou une baisse de biomarqueurs cardiaques, de préférence la troponine, avec au moins une valeur supérieure au $99^{\text {ème }}$ percentile, associée à des signes d'ischémie myocardique, avec au moins un des éléments suivants: une symptomatologie d'ischémie, une modification électrique significative indiquant une nouvelle ischémie, une onde $Q$ nouvellement apparue ou une preuve par imagerie, preuve d'une nouvelle perte de myocarde viable ou d'une anomalie de la contractilité régionale de la paroi myocardique vue à l'examen d'imagerie ${ }^{2}$.

Ce rapport propose une classification clinique des différents types d'infarctus du myocarde, reconnaissant 5 catégories distinctes de dommage myocardique sur la base de la physiopathologie ${ }^{2,3}$ :

- Type I: spontanément lié à une ischémie par évènement coronarien due à une érosion et/ou à une rupture, une fissuration ou une dissection de plaque,

- Type II: secondaire à une ischémie liée soit à une augmentation de la demande en oxygène, soit à une diminution de l'apport en oxygène,

- Type III : mort subite,

- Type IV : (a) sur angioplastie, (b) sur thrombose de stent,

- Type V : après pontage coronarien chirurgical. 


\section{B. Physiopathologie des dommages myocardiques post opératoires}

Le terme de "dommages myocardiques" regroupe de nombreuses entités pouvant aller de la nécrose myocardique histologique de quelques cellules à l'infarctus étendu. Plus particulièrement, deux mécanismes, isolés ou associés, ont été identifiés dans la survenue d'un infarctus du myocarde post opératoire : une ischémie myocardique prolongée par un déséquilibre de la balance entre la demande et les apports en oxygène et, plus rarement, une thrombose coronaire au niveau d'une plaque d'athérome ${ }^{4}$.

En per et post opératoire, plusieurs éléments peuvent être impliqués dans le déséquilibre de la balance myocardique en oxygène ${ }^{5}$. Ainsi, les épisodes de tachycardie, d'hypertension, l'hypothermie sont des éléments générant une dette en oxygène. D'autres facteurs, tels qu'une hypoxémie, une anémie ou encore une hypotension vont, eux, limiter l'apport en oxygène. Enfin, l'inflammation et l'hyper coagulabilité péri opératoire qui sont associés, contribuent à aggraver ce déséquilibre.

On comprend ainsi que le type II soit la forme majoritairement retrouvée en post opératoire de chirurgie non cardiaque, plus rarement le type $\mathrm{I}^{6}$.

II existe un continuum temporel et étiologique entre l'état cardiaque pré opératoire, le déroulement per opératoire et les complications cardiaques post opératoires $^{7}$. La réserve coronarienne, stable en pré opératoire, est diminuée par les différents éléments pouvant déséquilibrer la balance en oxygène. Ils entrainent une ischémie pouvant être marquée par un sous-décalage du segment ST, entrainant à son tour des lésions non transmurales par altération du réseau micro-circulatoire ${ }^{8}$. Ces lésions sont moins profondes que celles observées lors des infarctus médicaux par thrombose coronaire, plus étendues et non systématisées. Elles font le lit des infarctus du myocarde post opératoires (IMPO) ${ }^{9}$.

Du fait des différentes présentations de lésion myocardique, pouvant aller des formes frustres et atypiques, aux formes plus étendues voire à l'infarctus du myocarde massif, nous parlerons ici de dommage myocardique post opératoire (DMPO) afin de regrouper toutes ces entités nosologiques. 
La cinétique d'élévation de la troponine est différente selon le type de lésion ${ }^{9}$. Le Manach et al. dans une étude regroupant 1136 patients opérés d'une chirurgie aortique abdominale montraient 3 types de $\mathrm{DMPO}^{10}$ (figure 1). D'après les auteurs, les cinétiques de troponine sur les 3 premiers jours post opératoires peuvent être interprétées ainsi :

- Le premier type (14\% des patients) correspondait à des DMPO précoces survenant essentiellement entre la $6^{\text {ème }}$ et la $48^{\text {ème }}$ heure post opératoire. II correspond, par l'élévation brutale de troponine, à une thrombose aigue sur sténose pré existante,

- Le deuxième type (21\% des patients) représentait des DMPO plus tardifs généralement précédés par des valeurs modérément anormales (entre 0,2 et $1 \mathrm{ng} / \mathrm{ml}$ ) pendant $24-48 \mathrm{~h}$,

- Le troisième type (64\% des patients) était marqué par une élévation de troponine sur les 4 premiers jours post opératoires correspondant à des épisodes d’ischémie répétés.

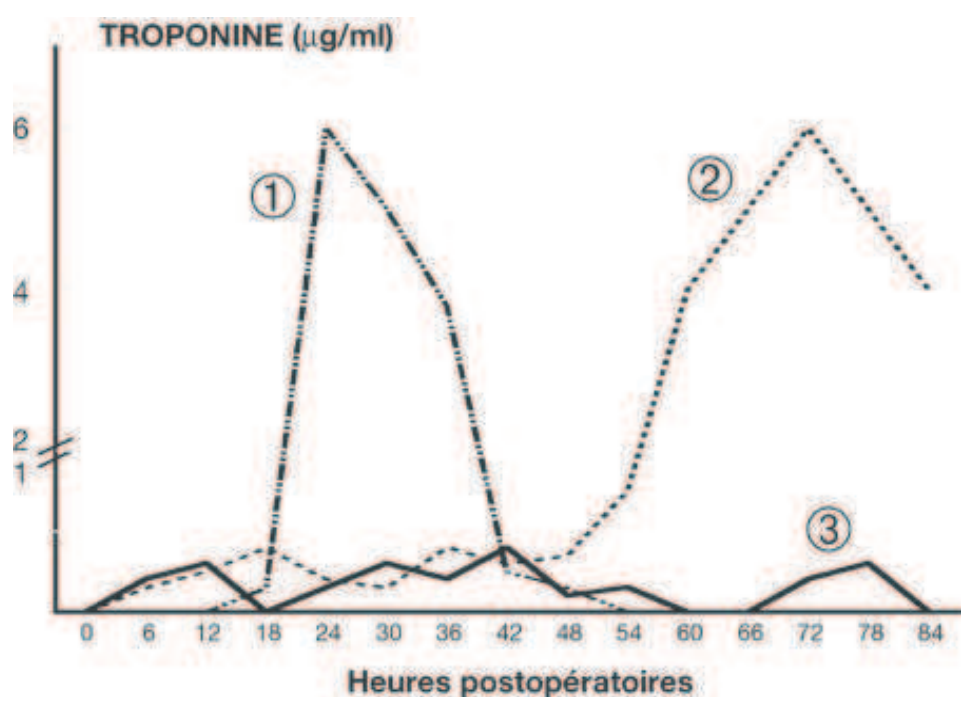

Figure 1 : cinétiques de troponine Ic dans 3 types de DMPO : 1) DMPO précoce, 2) DMPO retardé, faisant suite à des nécroses myocardiques à minima mais répétées, 3) DMPO à minima sans évolution vers l'infarctus massif d'après Coriat et al. $2005^{11}$.

La troponine peut donc s'élever très précocement en post opératoire. Dans cette même étude de Le Manach et al. ${ }^{10}$, I'IMPO était défini comme une troponinémie supérieure à $1,5 \mathrm{ng} / \mathrm{ml}$. L'élévation était détectable à partir de $37 \mathrm{~h} \pm 22 \mathrm{~h}$ dans les IMPO précoces avec un pic à $51 \mathrm{~h} \pm 30 \mathrm{~h}$, et une détection à partir de $74 \mathrm{~h} \pm 39 \mathrm{~h}$ dans les formes tardives avec un pic à $92 \mathrm{~h} \pm 34 \mathrm{~h}^{10}$ (figure 2). Dans l'étude de Godet et al. ${ }^{12}$ 
cherchant à trouver une valeur seuil de troponine permettant de prédire les complications cardio-vasculaires avant la sortie et à un an en chirurgie vasculaire, la troponine s'élevait dès la $3^{\text {ème }}$ heure post opératoire dans les formes précoces avec un pic de troponine entre la $12^{\text {ème }}$ et la $16^{\text {ème }}$ heure post opératoire. L'étude de Bursi et al. $^{13}$, dont l'objectif était d'évaluer le rôle pronostique de la stratification pré opératoire des patients de chirurgie vasculaire, retrouvait des résultats similaires.
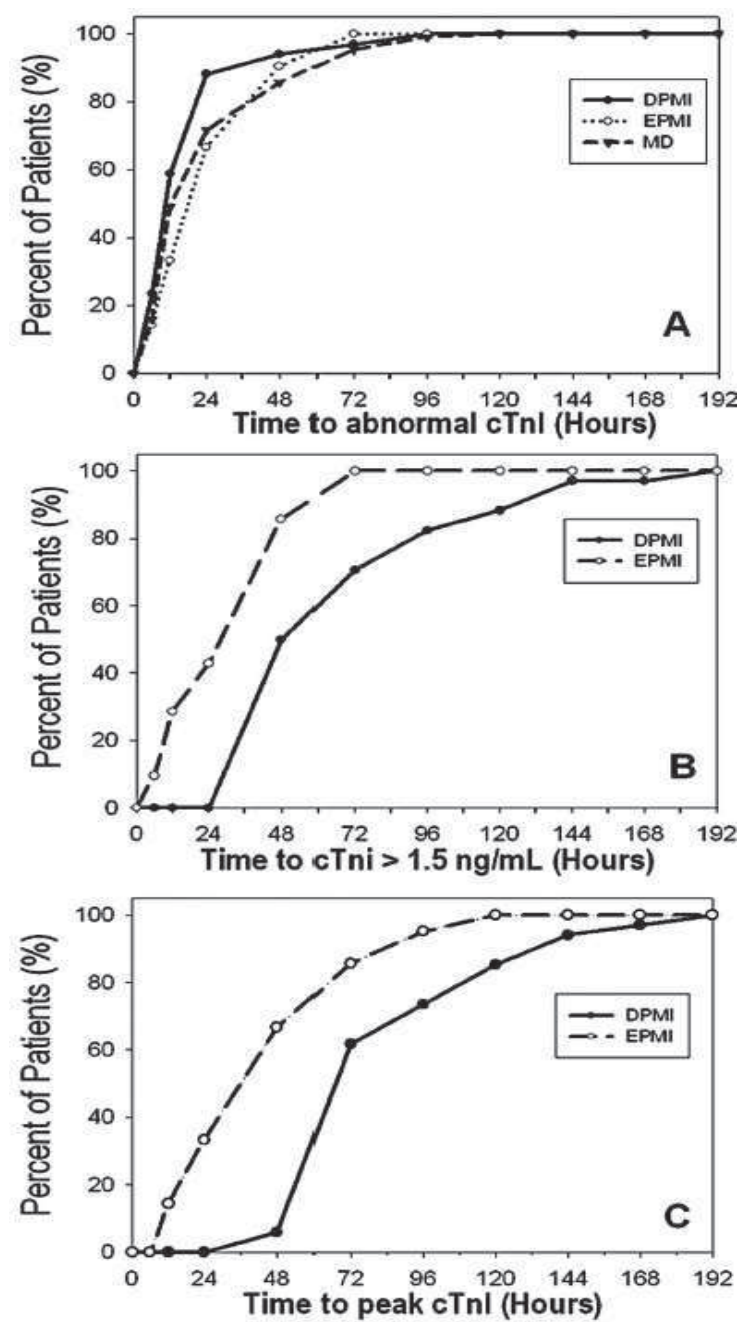

Figure 2 : profils de troponine Ic en fonction du temps depuis l'admission en soins intensifs jusqu'à un DMPO. A) délai jusqu'à la détection de la $1^{\text {ère }}$ valeur anormale de troponine Ic, B) délai jusqu'à l'IMPO (troponine Ic $\geq 1,5 \mathrm{ng} / \mathrm{ml}$ ), C) délai jusqu'au pic de troponine Ic, d'après Le Manach et al. $2005^{10}$.

D'autres travaux se sont intéressés à la durée d'ischémie précédant une élévation de la troponine. Dans une étude de Landesberg et al. ${ }^{14}$, 20,5\% des 185 patients programmés pour une chirurgie vasculaire avaient présenté au moins un évènement ischémique défini comme un sous décalage du segment ST. 6,5\% de ces patients avaient présenté un IMPO (défini comme une élévation de troponine I supérieure à $3,1 \mathrm{ng} / \mathrm{ml}$ associée à une symptomatologie clinique ou une modification électrique 
évocatrice). La durée moyenne d'ischémie des patients ayant développé un IMPO était de 226 \$164 minutes. En comparaison, les patients ayant présenté une ischémie simple, sans IMPO associé, avaient une durée moyenne d'ischémie plus courte de $38 \pm 26$ minutes.

\section{Incidence des dommages myocardiques post opératoires}

En chirurgie non cardiaque, les DMPO touchent entre 0,5 et 1,5\% avec 2 à $3,5 \%$ de complications cardiaques non létales ${ }^{15,16}$. Dans certaines chirurgies à haut risque de complication cardiaque telle que la chirurgie orthopédique pour fracture de hanche, on constate entre 20 et $30 \%$ d'élévation de troponine en post opératoire avec une mortalité à moyen et long terme particulièrement élevée ${ }^{17}$.

II a été constaté, selon les études, entre $12 \%$ et $47 \%$ d'élévation de troponine en post opératoire, tout type de chirurgie vasculaire confondue ${ }^{18}$. Dans l'étude de Brown et al. ${ }^{19}, 40 \%$ de lésions myocardiques, définies comme IMPO ou angor instable, ont été constatées chez 173 patients programmés pour une revascularisation des membres inférieurs ou cure d'anévrysme aortique abdominal programmé. L'étude de Guerre-Berthelot et al. ${ }^{20}$ rapporte 3 à $5 \%$ d'IMPO, observés après 54 chirurgies aortiques. De même, dans l'étude de Feringa et al. ${ }^{21}$ s'intéressant à la survie des patients opérés de chirurgie vasculaire majeure en contrôlant leur fréquence cardiaque par l'administration de béta bloquants, les auteurs avaient constaté $16,2 \%$ d'élévation de troponine et $31 \%$ d'ischémie myocardique définie comme une augmentation du biomarqueur ou une modification électrique.

Une élévation de troponine péri opératoire, quelle que soit son importance, grève le pronostic des patients. Toute élévation traduit une souffrance myocardique au niveau histologique avec diminution du capital myocardique ${ }^{9}$. C'est un facteur indépendant de complications cardio-vasculaires. 


\section{Retentissement pronostique des dommages myocardiques post opératoires}

\section{Troponinémie et complications cardiaques}

Les élévations de troponine en post opératoire sont associées à une augmentation de l'incidence des complications cardio-vasculaires ${ }^{22}$.

Dans l'étude de Le Manach et al. $^{10}$, les auteurs constataient que $75 \%$ des patients qui élevaient leur troponine développaient un IMPO à la $24^{\text {ème }}$ heure. De même, dans l'étude de Bolliger et al. ${ }^{22}$, 4,5\% d'évènements cardiaques dans les 30 jours post opératoires étaient retrouvés chez ceux ayant présenté une élévation de troponine parmi 133 patients opérés de chirurgie vasculaire. Dans la méta analyse de Redfern et al. ${ }^{23}$ s'intéressant au pronostic des patients de chirurgie vasculaire, les auteurs observaient que $21 \%$ de patients développaient au moins un évènement cardio-vasculaire à 18 mois parmi ceux ayant présenté une augmentation de troponinémie. Ces évènements cardiaques associés aux élévations de biomarqueur participent à l'augmentation du nombre de jours d'hospitalisation de ces patients ${ }^{8}$.

\section{Troponinémie et mortalité post opératoire}

Une étude récente de 2012 a trouvé une corrélation significative entre le pic de troponine T ultra-sensible mesuré dans les 3 premiers jours post opératoires et la mortalité à 30 jours en chirurgie non cardiaque ${ }^{24}$. La mortalité à 30 jours post opératoires était de 1,9\%. Les auteurs observaient $11,6 \%$ d'élévations de troponine ultra-sensible supérieure à $0,02 \mathrm{ng} / \mathrm{ml}$ et la mortalité augmentait avec la valeur du pic de troponine : un pic inférieur à $0,01 \mathrm{ng} / \mathrm{ml}$ était associé à une mortalité de $1 \%$, un pic à $0,02 \mathrm{ng} / \mathrm{ml}$ à une mortalité de $4 \%$, un pic compris entre $0,03 \mathrm{ng} / \mathrm{ml}$ à $0,29 \mathrm{ng} / \mathrm{ml}$ à une mortalité à $9,3 \%$, et un pic supérieur à $3 \mathrm{ng} / \mathrm{ml}$ avec une mortalité de 16,9\%.

La chirurgie vasculaire est une chirurgie à haut risque de mortalité avec un taux de décès entre 3 et $5 \%$, pratiquement toujours d'origine cardio-vasculaire ${ }^{17}$. En effet, plusieurs études rapportent une forte relation entre élévation de troponine et mortalité cardio-vasculaire en chirurgie non cardiaque, aussi bien à court qu'à long termes ${ }^{25,26}$. En post opératoire immédiat, Le Manach et al. ${ }^{10}$ rapportaient une mortalité corrélée au délai de survenue de DMPO : $24 \%$ dans les formes précoces, 
$21 \%$ dans les formes retardées, $7 \%$ dans les formes décrites comme des épisodes d'ischémies répétés, contre 3\% dans la population sans élévation de troponine. Dans la méta-analyse de Redfern et al. ${ }^{23}$ la mortalité à 30 jours, toute cause confondue, était de 2,3\% en l'absence d'élévation de troponine, de 11,6\% avec une élévation de troponine isolée et de 21,6\% avec un IMPO. Dans l'étude de Kim et al., les auteurs constataient $21 \%$ de décès à 6 mois dans le groupe ayant eu une élévation de troponine post opératoire contre $6 \%$ dans le groupe sans élévation de troponine ${ }^{27}$. Le risque de décès était alors multiplié par 6 si la troponinémie était supérieur à $3 \mathrm{ng} / \mathrm{ml}$. A un an, aucun seuil de troponinémie n'a été encore identifié comme facteur de risque de décès ${ }^{12}$ mais à long terme, la mortalité est toujours corrélée au pic de troponine, celle-ci étant multipliée par 2 les années suivant la chirurgie ${ }^{17}$ (figure 3). De plus, une étude récente de $2012^{28}$ dans la même population chirurgicale utilisant la troponine hypersensible comme facteur prédictif de mortalité, confirme une mortalité proportionnelle au pic de troponine ultra-sensible.

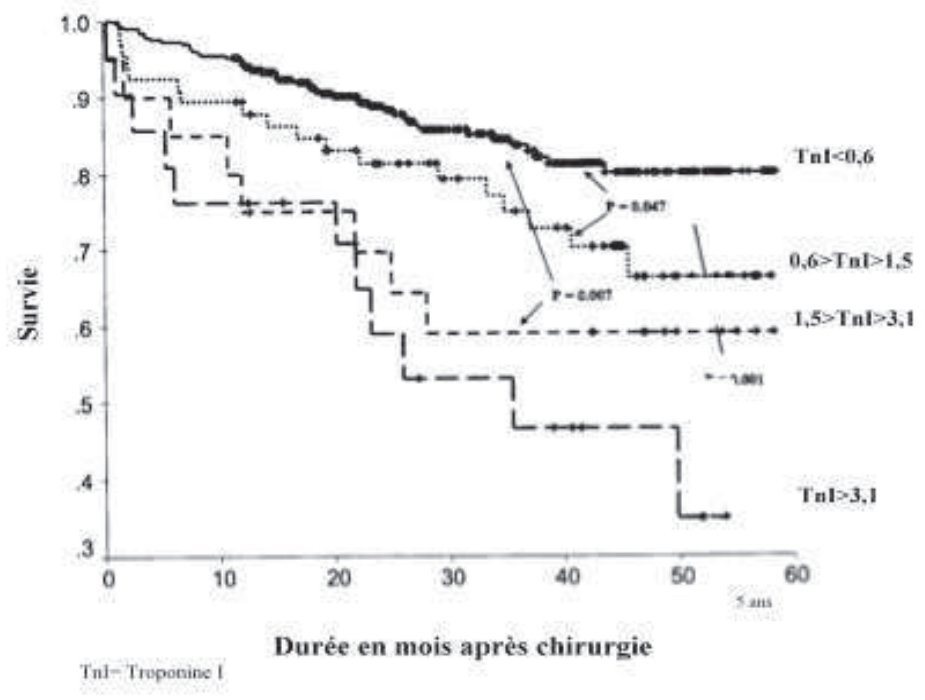

Figure 3 : Survie après un DMPO en fonction de la valeur de troponinémie post opératoire. Même les augmentations minimes de troponine prédisent une augmentation de la mortalité à long terme d'après Landesberg et al. $2003^{29}$.

La valeur péjorative de la survenue d'une élévation de troponine en post opératoire a généré de nombreux travaux destinés à évaluer le risque ischémique post opératoire d'après les données pré opératoires. 


\section{GESTION PERI OPERATOIRE DES DOMMAGES MYOCARDIQUES POST OPERATOIRES}

\section{A. Evaluation pré opératoire}

Le risque général d'évènement cardiaque péri opératoire en chirurgie non cardiaque chez les hommes de plus de 50 ans est d'environ $0,8 \%$, chiffre pondéré par les comorbidités, le statut cardio vasculaire, le type de chirurgie ${ }^{30}$. Du fait de l'importance du retentissement pronostique d'une élévation de troponine, la Société Française de Cardiologie (SFC) et la Société Française d'Anesthésie et de Réanimation (SFAR) ont proposé, en 2010, une stratification pré opératoire des patients selon un niveau de risque tenant compte de la chirurgie, de l'état cardiaque du patient et de ses antécédents ${ }^{17}$.

\section{Evaluation du risque lié à la chirurgie}

Les chirurgies thoracique et carotidienne représentent un risque intermédiaire avec 1 à $5 \%$ d'évènements péri opératoires alors que les chirurgies aortiques et vasculaires périphériques sont associées à un risque majeur de plus de $5 \%$ d'évènement péri opératoires.

\section{Evaluation du risque lié au patient}

Sont considérés à haut risque les patients présentant :

- Un syndrome coronarien aigu de moins d'un mois,

- Un angor invalidant ou une insuffisance cardiaque non compensée,

- Une dyspnée stade NYHA 3 ou 4 ,

- Des troubles du rythme cardiaque significatifs (fibrillation auriculaire, flutter à conduction ventriculaire rapide, extrasystoles ventriculaires polymorphes, salves de tachycardie ventriculaire) ou des troubles de conduction de haut grade (bloc trifasciculaire, bloc atrio ventriculaire de niveau 2 ou 3 ),

- Une valvulopathie mitrale ou aortique sténosante jugée sévère en échographie ou symptomatique (associée à une dyspnée NYHA 2). 
Sont considérés à risque intermédiaire les patients présentant :

- Une chirurgie programmée aortique, vasculaire majeure ou vasculaire périphérique,

- Une faible capacité physique (inférieure à 4 équivalents métaboliques (MET)) ou non évaluable,

- Des antécédents d'insuffisance cardiaque ou d'accident vasculaire cérébral,

- Un diabète, une insuffisance rénale, une anémie,

- Un âge avancé, facteur aggravant.

Sont considérés à faible risque les patients n'ayant pas de symptomatologie pour des activités physiques modérées ou intenses, ou un test fonctionnel maximal négatif récent.

Les examens complémentaires pré opératoires sont prescrits s'ils modifient l'attitude thérapeutique et/ou apportent un bénéfice à la prise en charge ultérieure ${ }^{17}$ (figure 4).

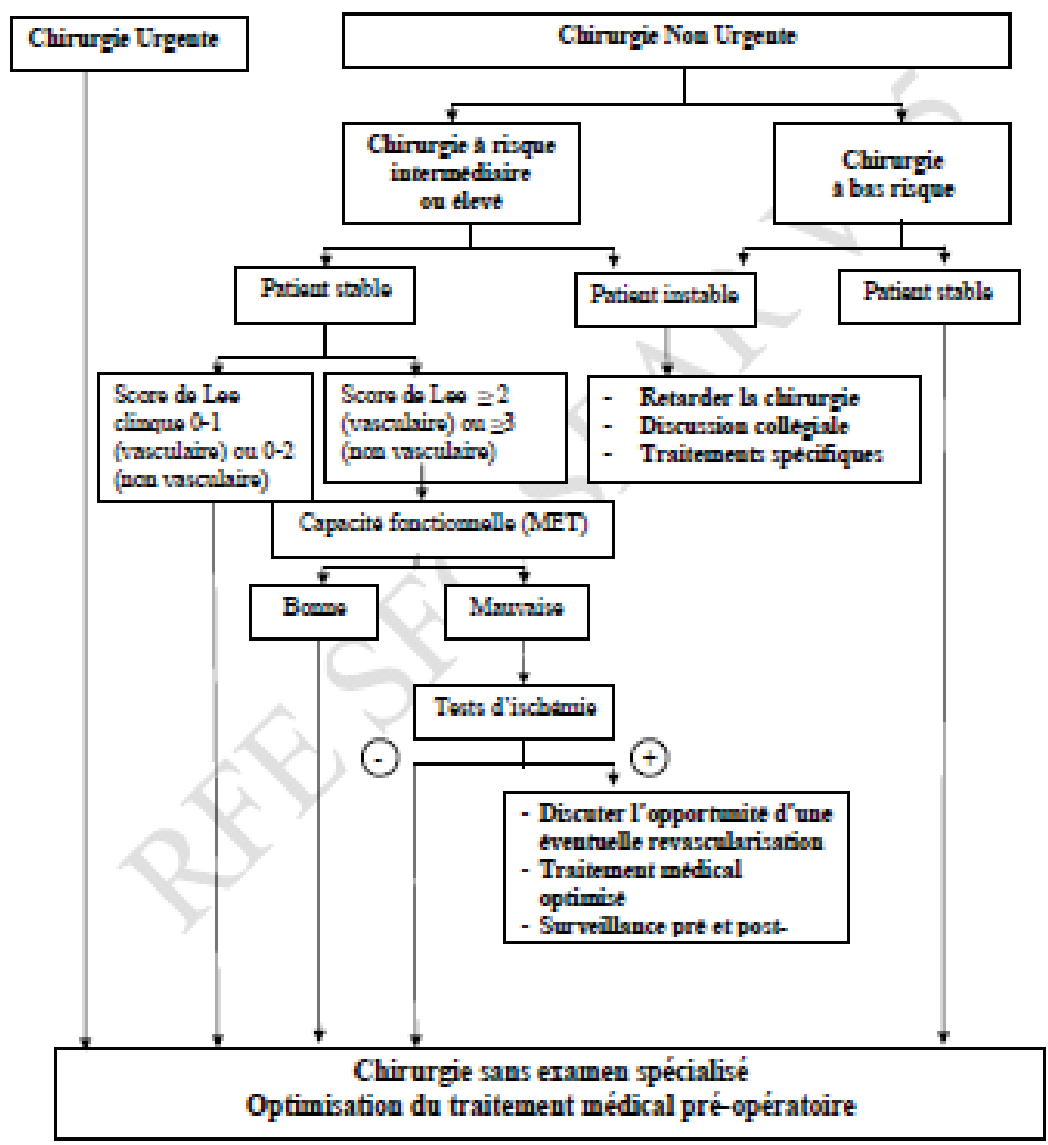

Figure 4 : algorithme général de prise en charge du coronarien avant chirurgie non cardiaque d'après les recommandations formalisées d'expert SFAR/SFC $2010^{17}$ 


\section{Score de Lee et Revised Cardiac Risk Index (RCRI)}

Le score de Lee représente le meilleur facteur pronostic actuel d'évènement cardiovasculaire péri opératoire basé sur le risque lié au patient. II associe un point à chaque pathologie : coronaropathie, insuffisance cardiaque congestive, insuffisance rénale avec élévation chronique de la créatinine, antécédent d'accident vasculaire cérébral ou d'accident ischémique transitoire, et diabète insulinodépendant. En intégrant le niveau de risque de la chirurgie au score de Lee, le Revised Cardiac Risk Index (RCRI) permet une meilleure stratification pré opératoire des patients selon leur niveau de risque d'évènement cardiaque majeur péri opératoire corrélé au score obtenu : 0 associé à un risque d'évènement de $0,4 \%, 1$ à $0,9 \%, 2$ à $6,6 \%$ et 3 ou plus à $11 \%{ }^{31},{ }^{32}$ (Annexe 1 : figure 24$)$.

\section{B. Evènements per opératoires}

La période per opératoire présente de nombreux facteurs déséquilibrant la balance en oxygène myocardique tels que :

- Les contraintes circulatoires,

- Le volume du saignement per opératoire,

- L'hypoxie,

- L'hypothermie.

L'anesthésiste doit s'attacher à limiter tous ces facteurs de risque d'ischémie myocardique. 


\section{Diagnostic des dommages myocardiques post opératoires}

\section{Symptômes et formes cliniques}

Les lésions myocardiques post opératoires sont asymptomatiques dans plus de $50 \%$ des cas et électriquement peu parlantes ${ }^{33}$. En effet, les DMPO de type II étant la forme majoritaire en post opératoire, il existe une prédominance des formes sous-endocardiques sans onde $Q$ séquellaire. Par ailleurs, la douleur n'est présente que dans $30 \%$ des cas du fait des antalgiques prescrits en per et post opératoires ${ }^{17}$.

Les dommages myocardiques sont alors le plus souvent révélés par une élévation de troponine lors de la surveillance post opératoire, et en sous-estiment la fréquence ${ }^{34}$.

\section{Evolution des biomarqueurs cardiospécifiques}

Les marqueurs de nécrose myocardique ont évolué depuis ces dernières années. La découverte de la troponine dans ses isoformes cardiospécifiques I et T a permis de remplacer les marqueurs historiques non cardiospécifiques tels que les CPK et la myoglobine ${ }^{35}$. Depuis 1999 , elle est devenue le biomarqueur diagnostique de référence des souffrances myocardiques et a été intégrée à la définition de l'infarctus du myocarde depuis $2000^{36}$.

Une élévation de troponine est définie par rapport au $99^{\text {ème }}$ percentile de la population générale asymptomatique ${ }^{37}$. Un coefficient de variation ou imprécision tolérable inférieur de $10 \%$ y est associé ${ }^{38}$. Sa détection est immunologique. Sa cinétique s'élève dans les 6 heures après lésion myocardique puis décroit de manière progressive sur plusieurs semaines.

Depuis quelques années, l'émergence des troponines ultra-sensibles permet un diagnostic plus précoce. 


\section{Place de la troponine T ultra-sensible}

La cinétique de la troponine $\mathrm{T}$ ultra-sensible est plus rapide que celle de la troponine classique. Son taux s'élève à partir de la $3^{\text {ème }}$ heure après une lésion myocardique $^{39}$. Sa forte valeur prédictive négative permet d'exclure précocement les douleurs thoraciques non ischémiques aux urgences ${ }^{40}$ et les cycles de dosage permettent de stratifier les patients à risque de nécrose myocardique ${ }^{41}$. Elle permet un diagnostic plus précoce par rapport aux troponines classiques du fait d'une meilleure sensibilité au dépend d'une moins bonne spécificité ${ }^{42}$. Les formes ultrasensibles permettent ainsi une prise en charge précoce dans les syndromes coronariens aigus.

L'interprétation d'une élévation de troponine ultra-sensible suit 3 principes ${ }^{43}$ :

- Toute élévation supérieure au $99^{9}$ ème percentile a une signification pronostique péjorative avec une augmentation de la mortalité à 5 ans,

- Toute augmentation ne signifie pas une thrombose aigue coronaire mais une lésion myocardique au sens histologique,

- L'orientation diagnostique est faite selon le contexte clinique.

\section{Prise en charge des dommages myocardiques en post opératoire : recommandations actuelles}

Etant donné le retentissement pronostique de toute élévation de troponine en post opératoire, le diagnostic précoce d'un DMPO devrait permettre l'instauration d'un traitement précoce.

Une surveillance continue du segment ST en post opératoire suppose une hospitalisation en unité de soins continus, étant donné l'implication des épisodes d'ischémie prolongée dans la formation des lésions sous-endocardiques, le plus souvent silencieuses.

En cas d'élévation de troponine en post opératoire orientant vers une complication cardiaque, un arbre décisionnel a été proposé selon le niveau de risque dont relève le patient (figure 5$)^{33}$ : 


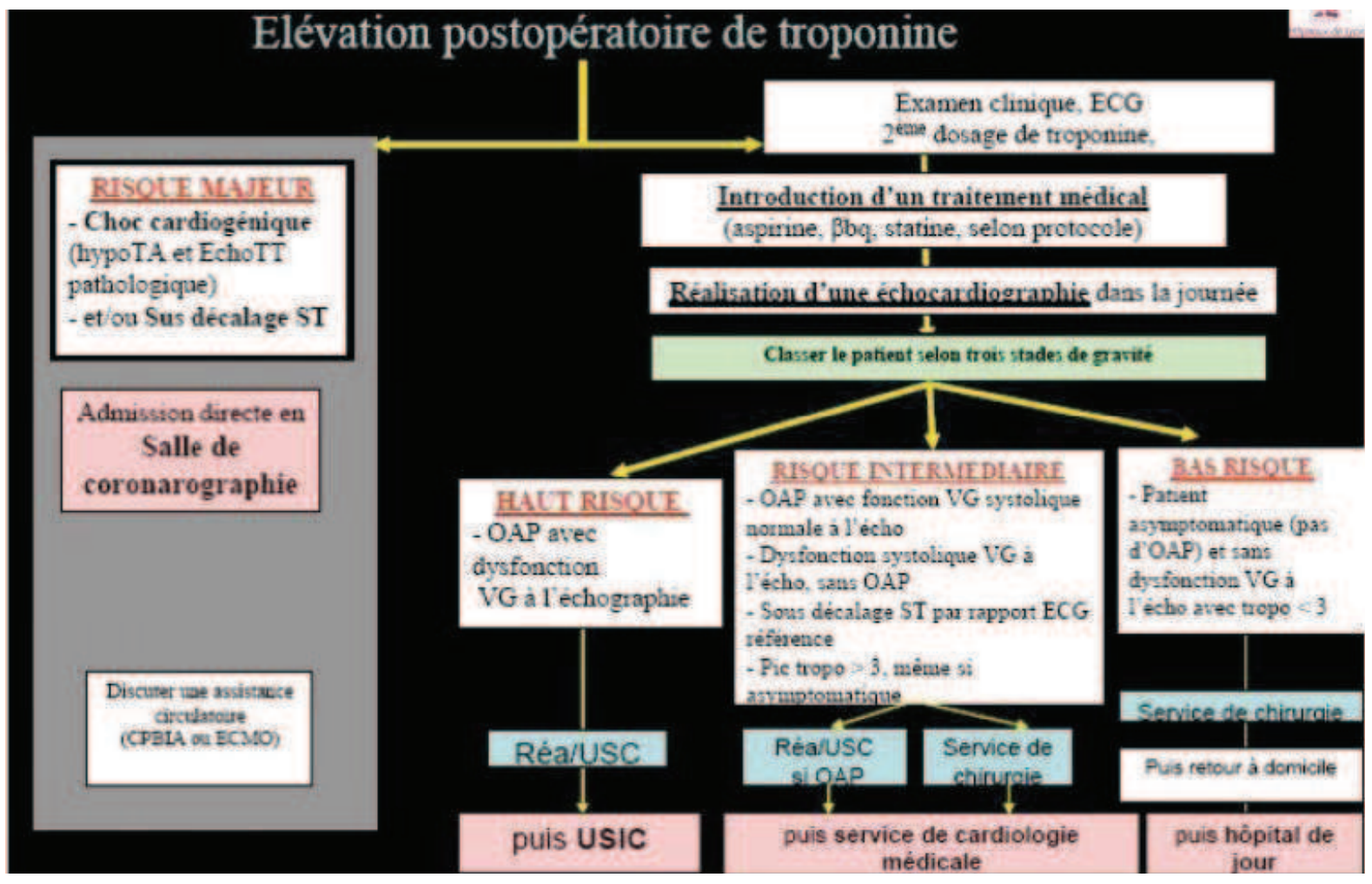

Figure 5 : algorithme de prise en charge actuel des élévations de troponine en post opératoire d'après Piriou3.

La prise en charge est extrapolée à partir des recommandations pour le traitement des syndromes coronariens aigus médicaux. II est étiologique et spécifique, après évaluation de la balance bénéfice - risque dans le contexte post opératoire et concertation multidisciplinaire ${ }^{3,17}$. 


\section{PLACE DE L'IMAGERIE DANS L'EVALUATION DU DMPO}

\section{A. Territoires d'ischémie myocardique et importance de la micro- vascularisation}

Un tiers des accidents coronariens post opératoires surviennent dans des territoires en l'absence de lésion sur les troncs épicardiques. En effet, les sténoses critiques sont rarement impliquées et le site d'infarcissement est indépendant de la zone de sténose pré opératoire ${ }^{17}$. Afin de mieux comprendre la physiopathologie de ces DMPO, les études post-mortem se sont attachées à établir le poids des sténoses coronariennes pré opératoires et des facteurs déstabilisant la balance en oxygène dans le mécanisme étiologique de formation des DMPO.

II est souvent pensé que les étiologies de DMPO sont également réparties entre rupture de plaque et déséquilibre de la balance en oxygène ${ }^{44,45,46}$. Par exemple, dans l'étude de Dawood et al ${ }^{44}, 55 \%$ des patients décédés d'un IMPO présentaient une fissure, rupture ou hémorragie de plaque contre $40 \%$ chez ceux décédés d'un infarctus du myocarde d'origine médicale. Malgré ces constatations, aucune relation causale n'a pu être établie entre les lésions et la cause de l'IMPO. De même, dans l'étude de Cohen et al. ${ }^{45}$, les IMPO dus à une rupture de plaque étaient répartis de manière aléatoire sur les 17 jours post opératoires alors que les lésions dues à un déséquilibre de la balance prédominaient dans les $1^{\text {ers }}$ jours post opératoires. Des $56 \%$ des patients présentant une thrombose coronaire, seulement $19 \%$ sont décédés. Plus particulièrement en chirurgie vasculaire, l'étude de Paul et al. ${ }^{46}$ comparant les données de la coronarographie pré opératoire aux ischémies myocardiques en post opératoire de chirurgie vasculaire, une sténose coronaire très serrée (supérieure à 90\%) n'était pas retrouvée en amont du territoire nécrosé orientant vers l'importance d'un réseau de collatéralité. La synthèse des études post mortem de Biccard et al. ${ }^{4}$ suggère que les hypoperfusions sur sténoses coronaires, impliquées dans le déséquilibre de la balance en oxygène, auraient un poids plus important dans la formation des DMPO que la rupture de plaque. Ceci confirme l'importance du réseau de collatéralité bien que la protection soit incomplète ${ }^{4}$. 
Une meilleure compréhension de la physiopathologie des DMPO passerait donc par l'exploration du lit micro-circulatoire myocardique.

\section{B. La coronarographie, gold standard dans l'exploration des}

\section{cardiopathies ischémiques?}

La coronarographie est, à ce jour, la technique de référence dans le diagnostic et l'exploration de la macro-circulation des cardiopathies ischémiques. Elle permet également de réaliser un geste de revascularisation dans le même temps si cela s'avère nécessaire ${ }^{47}$. Elle permet d'établir la morphologie et le degré des sténoses ${ }^{48}$.

Cependant, il s'agit d'un acte invasif et irradiant exposant le patient à un certain nombre de complications ${ }^{5}$. Elle ne permet ni l'exploration de la microcirculation, ni l'analyse fonctionnelle du myocarde, facteurs impliqués dans la physiopathologie des DMPO. Elle est le plus souvent de réalisation impossible dans le contexte post opératoire. 


\section{Explorations fonctionnelles non invasives disponibles à l'heure actuelle en post opératoire}

Depuis quelques années, de nouvelles techniques dans l'exploration des cardiopathies ischémiques se sont développés ${ }^{49}$ :

- L'échocardiographie trans thoracique (ETT) : utilisant les ultrasons, elle permet d'accéder à une évaluation anatomique et fonctionnelle cardiaque. Elle est non invasive et non irradiante. Cependant, elle est limitée par la fenêtre acoustique du patient et est opérateur dépendante,

- Le coroscanner : utilisant les radiations ionisantes et les produits de contraste iodés, il permet de cartographier le réseau coronaire et d'en rechercher les sténoses. Mais il ne permet pas l'exploration du métabolisme myocardique,

- la ventriculographie isotopique et la tomographie par émission de positron (PET scan) : l'injection d'un produit radiologique isotopique permet d'évaluer le métabolisme myocardique. La scintigraphie nécessite l'utilisation répétée de rayons gamma et pose des problèmes de résolution spatiale. Le PET scan bien que très performant pose des problèmes de disponibilité et de coût,

- l'imagerie cardiaque par résonance magnétique (IRMC) : utilisant les champs magnétiques et non ionisante, elle permet, après synchronisation cardiaque, une évaluation morphologique, fonctionnelle, et métabolique du myocarde, sans être opérateur dépendant. Les images sont de hautes résolutions spatiale et temporelle. La reproductibilité est très bonne. L'injection de produit de contraste à base de gadolinium permet l'exploration de la micro-circulation et de donner un pronostic sur la viabilité myocardique.

Le PET scan est l'examen de référence dans l'exploration du métabolisme myocardique $^{50,51}$. La sensibilité et la spécificité de l'examen sont de $85 \%$ et $89 \%$ respectivement. Cependant, son caractère irradiant, la faible disponibilité de l'examen, et un coût important, limitent sa généralisation. L'IRM cardiaque fait figure de très bonne alternative dans l'exploration des cardiopathies ischémiques autant sur les plans anatomiques et fonctionnels, que métabolique, d'autant plus que l'examen n'est pas irradiant ni opérateur dépendant ${ }^{50}$ (figure 6 ). II existe une très faible variabilité inter et intra observateur dans l'évaluation des fonctions ventriculaires droite et gauche ${ }^{52}$. La résolution spatiale, supérieure aux autres explorations, permet 
une meilleure exploration du lit sous-endocardique impliqué dans la genèse des lésions myocardiques post opératoires ${ }^{53}$.

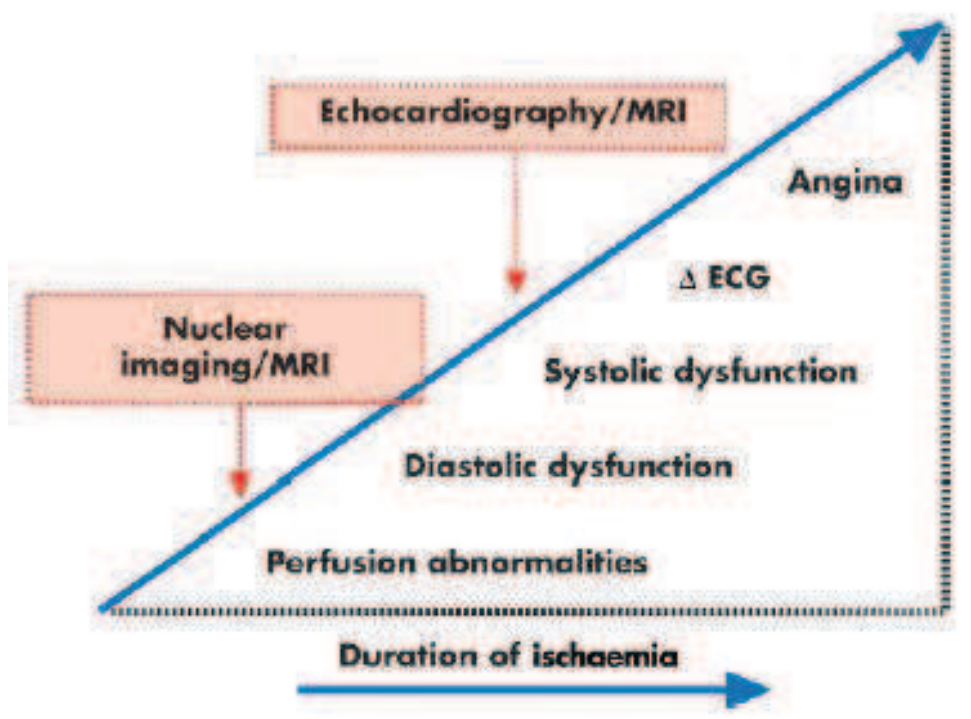

Figure 6 : « cascade ischémique » représentant les évènements faisant suite à une ischémie, d'après Yan et al.2006 ${ }^{61}$

\section{Principe de fonctionnement de l'IRM}

L'imagerie par résonance magnétique est un mode non invasif non irradiant d'imagerie. Les noyaux d'hydrogène des molécules d'eau se comportent comme des dipôles lors de l'application d'un champ magnétique et s'alignent dans l'axe du champ $^{30}$. L'application d'une onde de radiofréquence crée une déflection par rapport à l'axe du champ magnétique. Le signal est généré lors du retour des noyaux d'hydrogène à la position de repos: il s'agit des séquences T1 (figure 7). On reconstitue ainsi une image point par point avec une haute résolution spatiale en appliquant plusieurs ondes de radiofréquence.

L'examen peut être complété par l'injection intra-vasculaire de produit de contraste à base de gadolinium. Sa forme native s'accumule dans les parenchymes puis se distribue dans l'espace interstitiel où il se lie à l'albumine. Son élimination est rénale. Cet agent de contraste a été largement utilisé, à ses débuts, du fait de l'absence de limitation par la fonction rénale. 


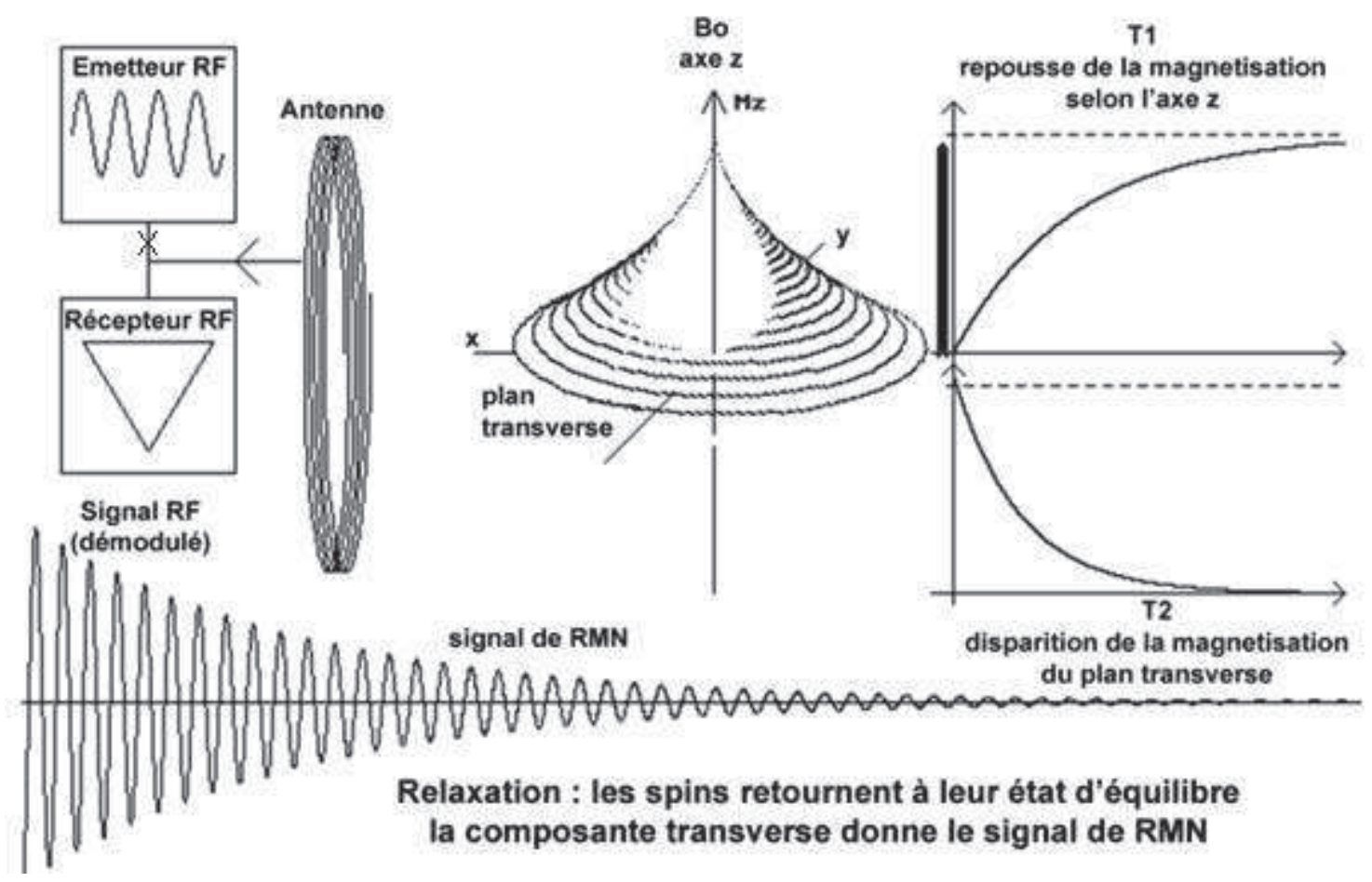

Figure 7 : émission du signal par la relaxation du spin d'hydrogène ${ }^{54}$

Depuis 2007 est apparue une complication rare mais potentiellement fatale : la fibrose systémique néphrogénique, majoritairement décrite chez les insuffisants rénaux ${ }^{55}$. Le gadolinium lié à l'albumine s'accumule dans les tissus et provoque un état inflammatoire et une fibrose touchant essentiellement le territoire cutané. Son évolution est chronique et peut s'étendre à d'autres organes tels que le poumon, le cœur, la dure mère. La déclaration de la maladie intervient entre quelques jours à 6 mois après l'exposition. II n'existe actuellement pas de traitement à part l'amélioration de la fonction rénale. 


\section{E. Déroulement d'une IRM cardiaque}

L'IRM cardiaque (IRMC) utilise la résonance magnétique en T1 pour obtenir des coupes du cœur (para sternale grand axe, para sternale petit axe, 4 cavités) permettant une exploration anatomique complète ${ }^{56}$. Les images nécessitent une synchronisation avec le rythme cardiaque, expliquant une limitation de la résolution de l'examen chez les patients présentant une arythmie rapide ou des extra systoles fréquentes.

L'examen dure environ 45 minutes (figure 8). Un temps d'apnée est nécessaire pendant la réalisation des séquences dynamiques. Les séquences réalisées commencent par des séquences sans gadolinium :

- séquences morphologiques: images fixes permettant la caractérisation tissulaire,

- si besoin, réalisation de séquences en T2 pour évaluation de l'œdème périlésionnel dans les accidents ischémiques aigus,

- séquences de fonction : exploration de la cinétique segmentaire, évaluation de la fraction d'éjection ventriculaire gauche, de l'épaisseur myocardique, du volume d'éjection systolique, du débit cardiaque et de la masse ventriculaire gauche,

- séquences de vélocimétrie : volume d'éjection systolique et débit cardiaque, pathologie valvulaire, shunts.

Ensuite, les acquisitions se poursuivent par l'injection de gadolinium :

- $1^{\text {er }}$ passage $^{57,58}$ : exploration de la micro-circulation myocardique et de la perfusion myocardique,

- Rehaussement tardif à 10 minutes et recherche d'un hyposignal intra rehaussement ${ }^{59}$ : évaluation de l'altération de la micro-circulation et détermination de la viabilité du myocarde (figure 9) (Annexe 2 : figures 26 et 27). 


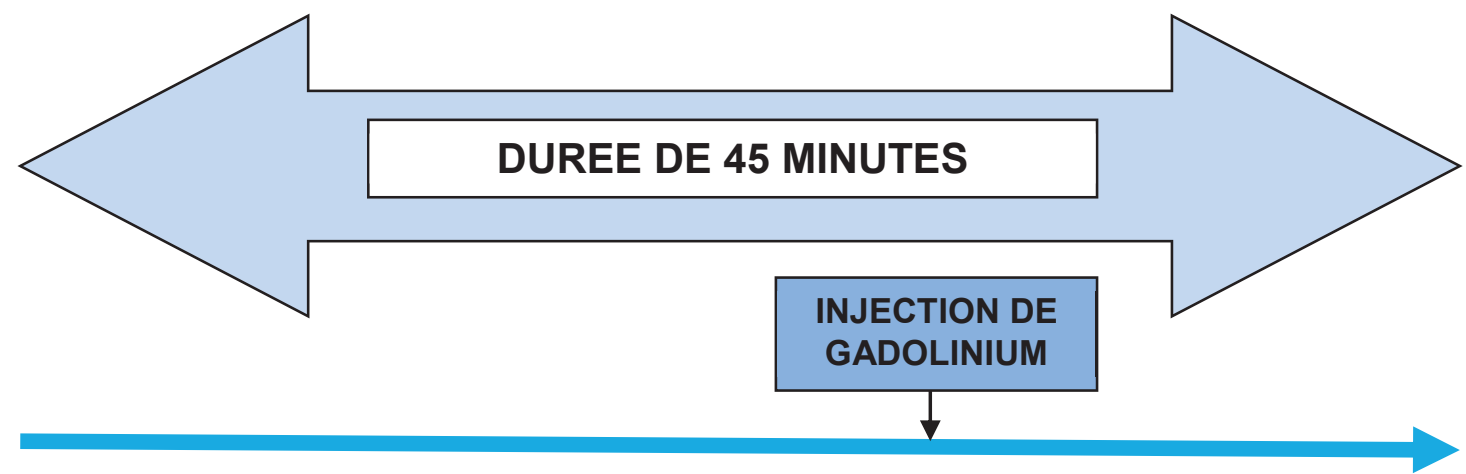

SEQUENCES DE MORPHOLOGIE, FONCTION ET VELOCIMETRIE $1^{\text {ER }}$ PASSAGE ET NO REFLOW, REHAUSSEMENT TARDIF

Figure 8 : déroulement d'une IRM cardiaque

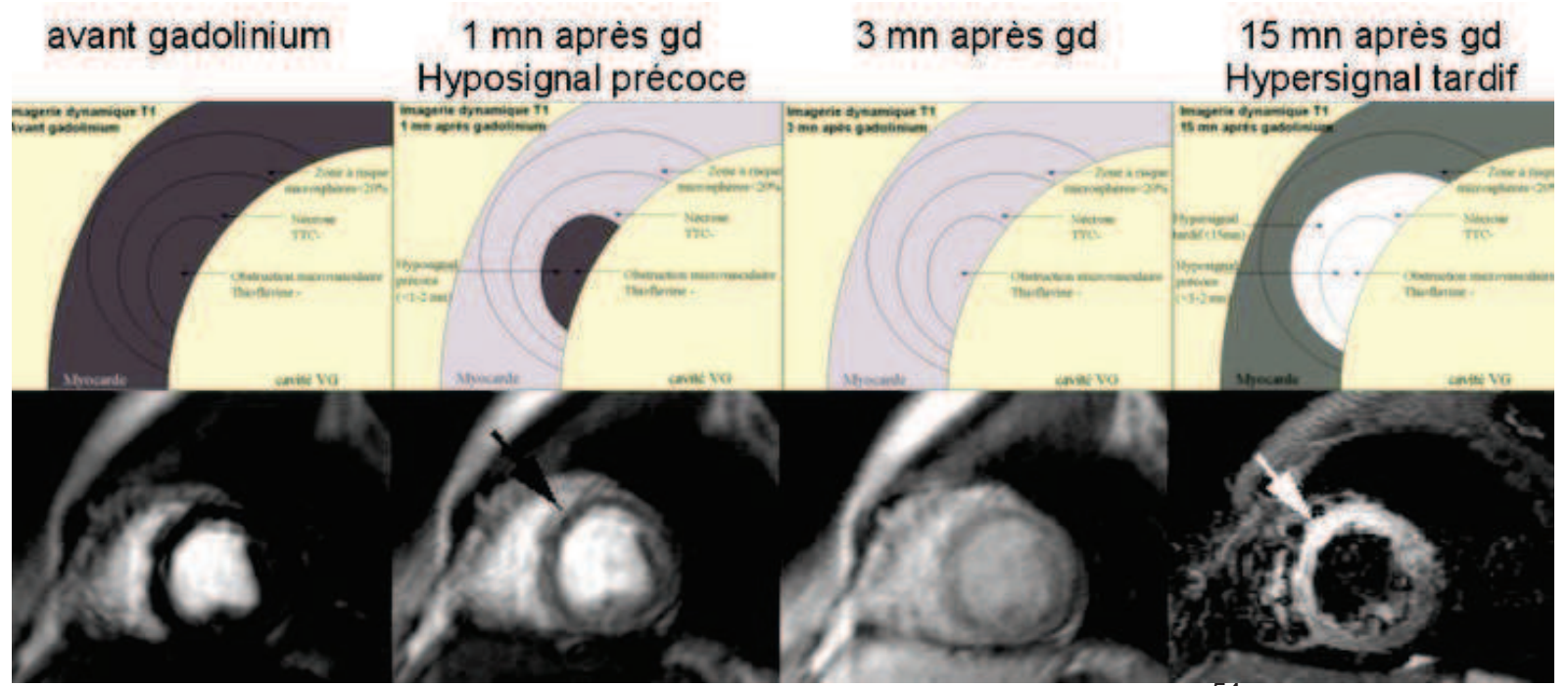

Figure 9 : évolution du gadolinium dans la paroi myocardique après injection d'après Germain et al. $2010^{54}$ 
La localisation des lésions se fait en découpant virtuellement le cœur en 17 segments pour faciliter le repérage lors de l'analyse des images (figure 10).

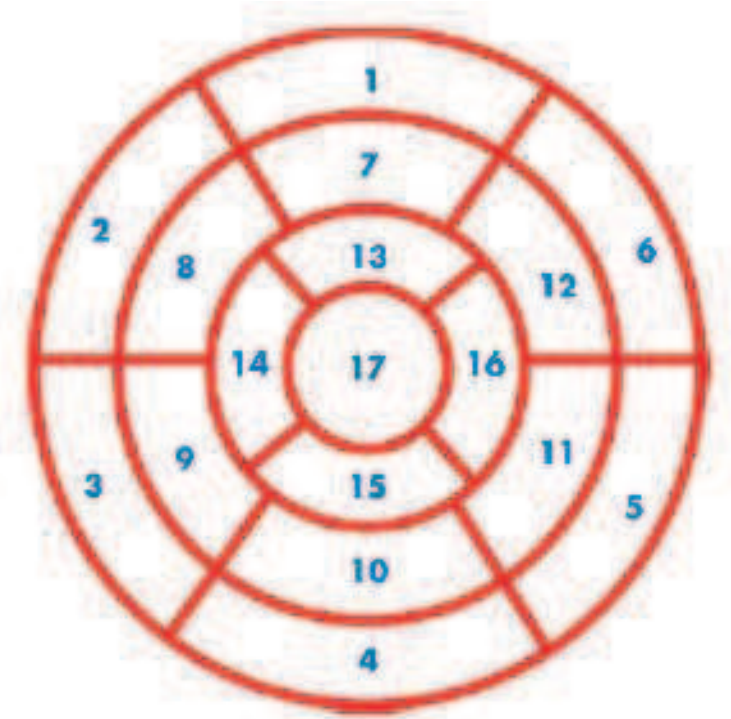

Figure 10 : représentation des 17 segments cardiaques utilisés en tomographie cardiaque, en échocardiographie et en IRM cardiaque, d'après Cerqueira et al. $2002^{60}$.

\section{F. Contre-indications a l'IRM}

Bien que non irradiante, l'IRM n'est pas sans risque et il existe des contreindications :

- Celles de I'IRM: la claustrophobie, la présence d'un pace maker ou défibrillateurs, la présence d'implants métalliques chirurgicaux magnétisables (clips vasculaires cérébraux, éclats métalliques oculaires),

- Celles de l'injection du produit de contraste à base de gadolinium: insuffisance rénale aigue ou chronique, allergie, anémies hémolytiques. 


\section{G. Principes d'interprétation d'une IRM cardiaque}

Avec l'injection d'agent de contraste, l'IRM cardiaque (IRMC) permet d'évaluer la perfusion myocardique sous-endocardique et la micro-circulation. Le gadolinium augmente le signal du myocarde perfusé.

Lors $\mathrm{du} 1^{\mathrm{er}}$ passage, les lésions sous-endocardiques et les défects transmuraux ne sont pas rehaussés, signant alors une altération précoce du flux sanguin $^{61}$ par altération de la micro-circulation ${ }^{62}$. Le gadolinium s'élimine du myocarde sain dans un délai d'environ 10 minutes.

Les lésions ischémiques présentent un retard à l'élimination du gadolinium du fait de l'altération de la micro-circulation. Le gadolinium est alors accumulé dans ces territoires altérés signalés par un hypersignal. Le pourcentage de transmuralité des lésions (ou épaisseur du myocarde atteint) permet d'évaluer le potentiel de récupération du myocarde et d'établir un pronostic sa viabilitée ${ }^{63}$. Une transmuralité maximale de $25 \%$ permet de présager d'une récupération myocardique ultérieure. On distingue ainsi les cicatrices fibreuses dont la transmuralité est de $100 \%$, sans potentiel de récupération, du myocarde hibernant pouvant toucher jusqu'à $25 \%$ de transmuralité ${ }^{52,64}$. L'évaluation du retentissement de l'ischémie myocardique sur la micro-circulation permet par exemple d'évaluer le bénéfice d'une revascularisation coronarienne avant réalisation du geste ${ }^{65,66}$.

L'IRMC bénéficie d'une très haute résolution spatiale permettant d'identifier des lésions à partir de $2 \mathrm{~g}$ de myocarde nécrosé ${ }^{67}$. C'est la méthode de choix dans l'exploration fonctionnelle et l'évaluation de la micro-circulation, permettant de visualiser le sous-endocarde où la présence de lésions peut signer une altération précoce du flux, celle-ci étant impliquée dans la formation de futurs infarctus transmuraux ${ }^{68}$. 


\section{H. Quantification des lésions}

La masse myocardique infarcie dépend de la durée de l'épisode ischémique et des possibilités de reperfusion de ce territoire. Le taux de troponine est directement corrélé au volume infarci ${ }^{69}$.

L'interprétation actuelle se fait selon 2 critères :

- D'une part, un nombre de segments atteints déterminant l'étendue de la zone lésée,

- D'autre part, un pourcentage de transmuralité déterminant la profondeur de paroi myocardique atteinte.

Les dommages myocardiques post opératoires sont, la plupart du temps, électriquement peu parlants, faiblement symptomatiques sur le plan clinique, corrélés à un pronostic défavorable, et majoritairement dus à des déséquilibres de la balance en oxygène. La compréhension des mécanismes physiopathologiques péri opératoires impliqués dans l'altération du lit micro-vasculaire sous-endocardique semble donc primordial. L'IRM cardiaque est la technique la plus performante et la plus disponible à l'heure actuelle, en dépit de son coût, pour l'exploration de la microcirculation myocardique et ainsi caractériser ces dommages myocardiques post opératoires, ce qui fait l'objet de ce travail. 
MATERIEL ET

METHODES 


\section{CADRE DE L'ETUDE}

\section{A. Type d'étude}

II s'agit d'une étude monocentrique réalisée au centre hospitalo-universitaire de Rouen au sein du service de chirurgie vasculaire et thoracique, observationnelle, descriptive, non randomisée, s'étant déroulée du 31 mars 2010 au 31 décembre 2011.

\section{B. Objectif principal}

Caractériser, en utilisant l'imagerie par résonance magnétique cardiaque, les dommages myocardiques en post opératoire de chirurgie vasculaire et thoracique chez les patients ayant présenté une élévation de troponine post opératoire.

\section{Objectifs secondaires}

- Incidence des dommages myocardiques en post opératoire dans le service de chirurgie vasculaire et thoracique,

- Comparaison des lésions objectivées à l'IRMC aux données cardiologiques pré et post opératoires disponibles,

- Analyse de la prise en charge des patients ayant présenté un dommage myocardique post opératoire,

- Suivi d'évolution à un an post opératoire des patients ayant bénéficié d'une IRM cardiaque.

\section{CRITERES D'INCLUSION ET D'EXCLUSION}

\section{A. Critères d'inclusion}

- Patients opérés de chirurgie vasculaire ou thoracique sous anesthésie générale ou loco régionale,

- Ayant bénéficié d'un dosage de troponine, standard Ic (Tnlc) ou ultra-sensible (TnTus) en post opératoire, dont la valeur est supérieure au $99^{\text {ème }}$ percentile. 


\section{B. Critères d'exclusion}

- Geste chirurgical à l'origine d'une élévation de troponine (ouverture péricardique), chirurgie en urgence,

- Refus,

- Troubles cognitifs, antécédents de démence

- Patients sous tutelle,

- Décès avant réalisation de l'IRM cardiaque,

- Présence d'une contre-indication à l'IRM ou à l'injection de gadolinium (clairance inférieure à $60 \mathrm{ml} / \mathrm{mn}$ ou non évaluable pour dénutrition sévère ou cachexie),

- Présence d'un élément entravant la précision de l'image :

- trouble du rythme rapide ou nombreuses extra systoles entravant la synchronisation cardiaque,

○ impossibilité de tenir le temps d'apnée.

\section{DEROULEMENT DE L'ETUDE}

Les cardiologues traitants ont été informés de l'étude en cours. Un ECG post opératoire était réalisé en cas d'élévation de troponine en post opératoire.

Le consentement de chaque patient était recueilli après annonce de l'élévation de troponine en post opératoire sur les dosages, avec explication des modalités de l'IRMC, explication de l'objectif de l'examen, et convocation en externe.

L'IRMC se déroulait en externe à distance de la chirurgie et suivait les séquences d'acquisition sur l'appareil IRM SYMPHONY 1,5 T - SIEMENS - 06. Les séquences étaient les suivantes :

- Séquences sans injection de gadolinium: séquences morphologiques, de fonction et de vélocimétrie,

- Séquences avec injection de gadolinium : $1^{\mathrm{er}}$ passage et rehaussement tardif.

L'évolution des patients à 1 an post opératoire a été obtenue par rappel téléphonique. 


\section{DONNEES RECUEILLIES}

Les valeurs du pic de troponine, le délai de ce pic par rapport au jour de l'intervention, ainsi que le type de chirurgie ont été recueillis chez l'ensemble des patients éligibles. Les autres données concernent les patients inclus.

\section{A. Données pré opératoires}

- Sexe, âge, indice de masse corporelle (IMC),

- Score de l'American Society of Anesthesiology (ASA),

- Capacité fonctionnelle (Annexe 1 : figure 25),

- Antécédents : HTA, hypercholestérolémie, tabagisme,

- Marqueurs des localisations de la maladie athéromateuse : RCRI,

- Indication chirurgicale et durée de chirurgie.

\section{B. Données per opératoires}

Les évènements per et post opératoires immédiats d'une durée supérieure à 10 minutes, étaient recueillis par l'étude des fiches d'anesthésie pour la période per opératoire et la surveillance post interventionnelle :

- Hypertension artérielle: définie comme une pression artérielle systolique supérieure à $140 \mathrm{mmHg}$ et/ou une pression artérielle diastolique supérieure à 90 $\mathrm{mmHg}$,

- Hypotension artérielle : définie comme une baisse de plus de $40 \%$ de la pression artérielle par rapport à la valeur initiale,

- Tachycardie: définie comme une fréquence cardiaque supérieure à 100 battements par minute,

- Anémie définie comme la nécessité d'une transfusion ou un taux d'hémoglobine inférieur au seuil transfusionnel fixé par la Haute Autorité de Santé : 7g/dL pour un patient sans antécédent particulier, 8 à $9 \mathrm{~g} / \mathrm{dL}$ pour un patient avec des antécédents cardio-vasculaires, $10 \mathrm{~g} / \mathrm{dL}$ en cas de mauvaise tolérance, d'insuffisance coronarienne aigue ou d'insuffisance cardiaque avérée ${ }^{70}$,

- Hypoxie définie comme une saturation pulsée en oxygène inférieure à $95 \%$ sous une fraction inspirée en oxygène de 100\%,

- Recours à des inotropes ou à des vasopresseurs : dobutamine ou noradrénaline ou association des 2. 


\section{Données post opératoires}

- Cinétique et valeur du pic de troponine post opératoire,

- Délai entre la chirurgie et le pic, J0 étant défini comme le jour de la chirurgie,

- Toute modification électrique entre les ECG pré et post opératoires (relecture avec l'aide d'un cardiologue),

- Délai de réalisation et conclusions de l'IRMC: nombre de segments, et transmuralité,

- Prise en charge post opératoire immédiate,

- Evolution des patients : décès, évènement cardio-vasculaire majeur.

Les données obtenues par IRMC ont ensuite été confrontées aux données des explorations cardiologiques pré et post opératoires disponibles. Après réflexion sur l'ensemble de ces données, nous avons tenté de déterminer si les lésions visualisées à l'IRM étaient des lésions anciennes ou nouvellement apparues dans ce contexte post opératoire. La localisation des lésions visualisées à l'IRM a été comparée aux territoires correspondant à la circulation coronaire, afin de déterminer l'existence d'une corrélation éventuelle avec des sténoses connues (figure 11).

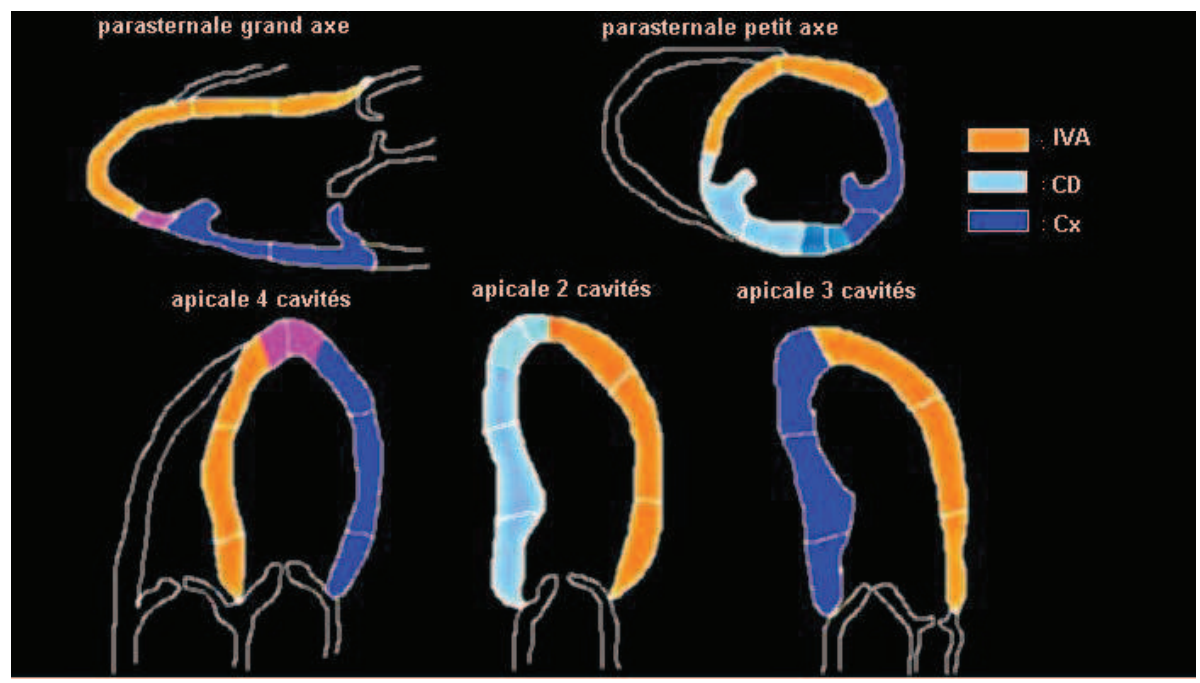

Figure 11 : territoires myocardique et vascularisation coronarienne d'après echorea.org ${ }^{71}$ 
RESULTATS DE L'ETUDE 


\section{DESCRIPTION DE LA POPULATION}

3145 patients ont été hospitalisés dans le service de chirurgie vasculaire et thoracique entre le 31 mars 2010 et le 31 décembre 2011.

\section{A. Patients ayant présenté une élévation de troponine}

Sur ces 21 mois d'inclusion, 1740 patients ont été opérés sous anesthésie générale ou loco régionale, pour une chirurgie vasculaire ou thoracique.

Sur ces 1740 interventions, urgences et chirurgies programmées, 249 patients ont présenté une élévation de troponine en post opératoire au-dessus du $99^{\text {gème }}$ percentile soit une incidence de $14,3 \%$.

Parmi ces 249 patients, on comptait 216 hommes pour 33 femmes soit un sexe ratio $\mathrm{H} / \mathrm{F}$ de 6,5 .

L'âge moyen de la population était de 71,7 ans.

40 patients avaient bénéficié d'un dosage de troponine lc sur 958 patients opérés entre le 31 mars 2010 et le 28 février 2011 (soit une incidence de 4,2\%), et 209 patients d'un dosage de troponine $T$ ultra-sensible sur 782 patients opérés entre le $1^{\text {er }}$ mars 2011 et le 31 décembre 2011 (soit une incidence de 26,7\%).

\section{Type de chirurgie}

Parmi ces 249 patients, la répartition du type de chirurgie était la suivante :

- 201 patients pour une chirurgie vasculaire (figure 12) : 56 chirurgies aortiques, 4 chirurgies carotidiennes, 96 interventions pour chirurgie vasculaire périphérique (thrombectomie ou angioplastie vasculaire), 29 amputations de membre inférieur, 9 abcès cutanés, 1 fistule artério veineuse, 1 pose de cathéter, 1 laparotomie exploratrice, 4 détersions de plaie,

- 48 patients pour une chirurgie thoracique (figure 13): 9 pneumonectomies, 20 lobectomies, 9 thoracoscopies, 6 médiastinoscopies, 2 bronchoscopies, 1 drainage pulmonaire, 1 tumorectomie cutanée. 


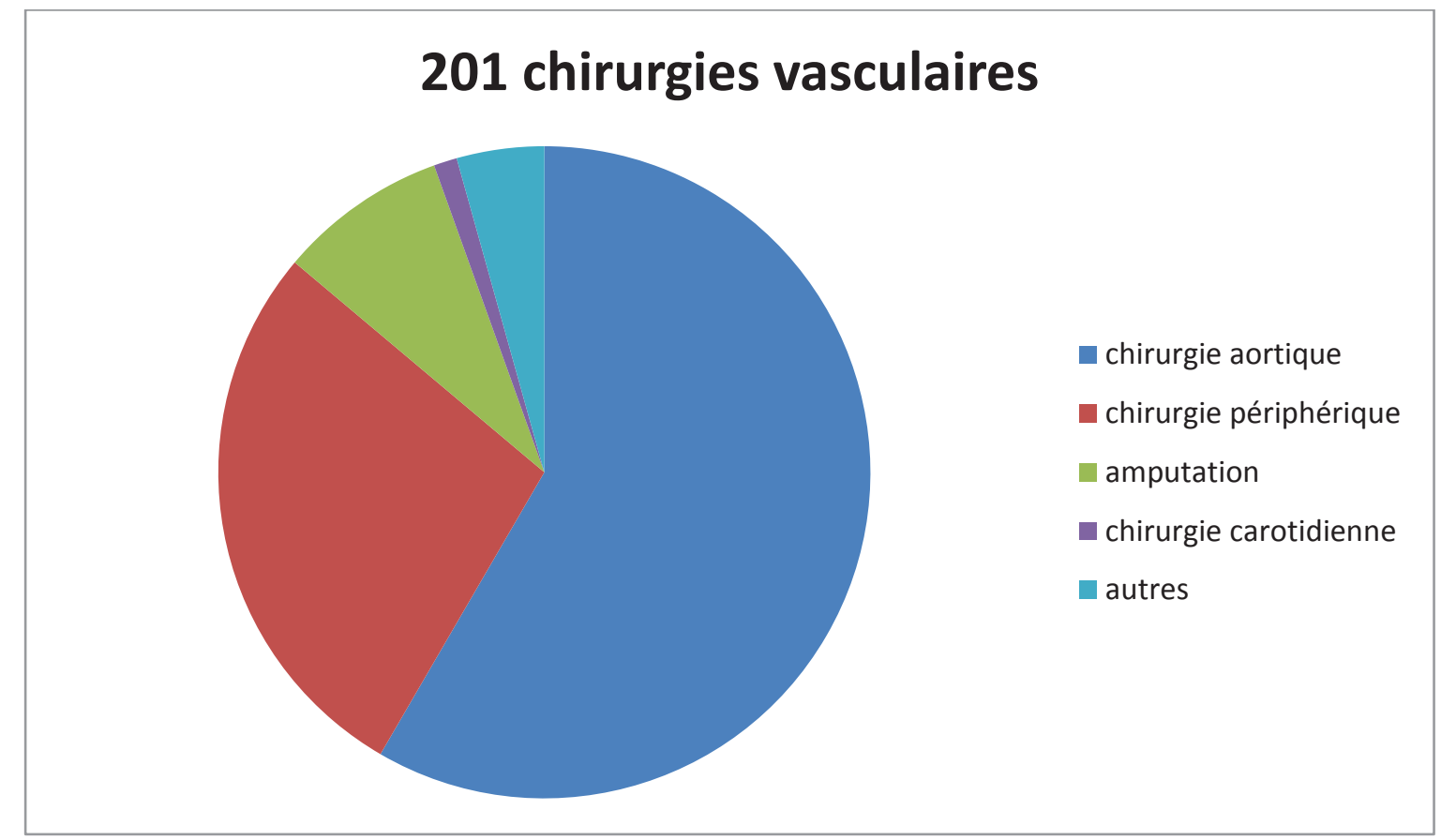

Figure 12 : répartition des indications chirurgicales vasculaires

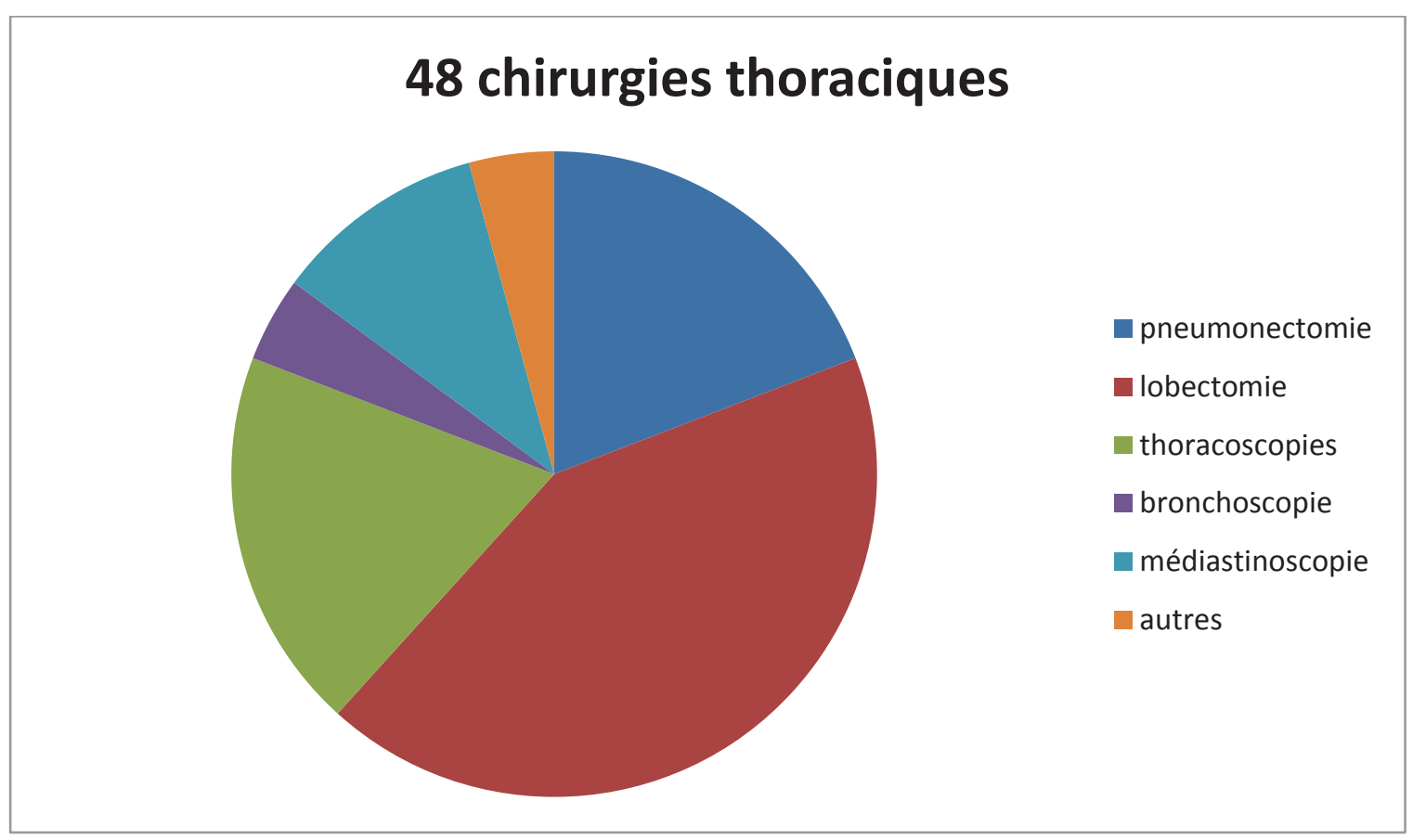

Figure 13 : répartition des indications chirurgicales thoraciques 


\section{Chronologie des pics de troponine}

Le type de dosage de la troponine a changé au cours de l'étude, passant d'une troponine I « standard » (Tnlc) à une troponine $T$ « ultra-sensible » (TnTus).

La majorité des pics de troponine sont survenus entre J0 (jour de l'intervention) et $\mathrm{J} 1$ (jour suivant l'intervention) (figure 14) : 83 patients à $\mathrm{J0}$ soit 33,3\%, 91 patients à $\mathrm{J} 1$ soit $36,5 \%, 19$ patients à $\mathrm{J} 3$ soit 7,6\%, 15 patients à $\mathrm{J} 4$ soit $6 \%, 6$ patients à $\mathrm{J} 5$ soit $2,4 \%$. Au-delà de ce délai, 35 patients ont présenté une élévation de troponine soit $14 \%$.

La moyenne du pic de Tnlc était de $21 \mathrm{ng} / \mathrm{ml}$ (ext. : 1,01 - $465 \mathrm{ng} / \mathrm{ml}$ ) et celle du pic de de TnTus de $174 \mathrm{ng} / \mathrm{ml}$ (ext. : 15 - $10986 \mathrm{ng} / \mathrm{ml}$ ).

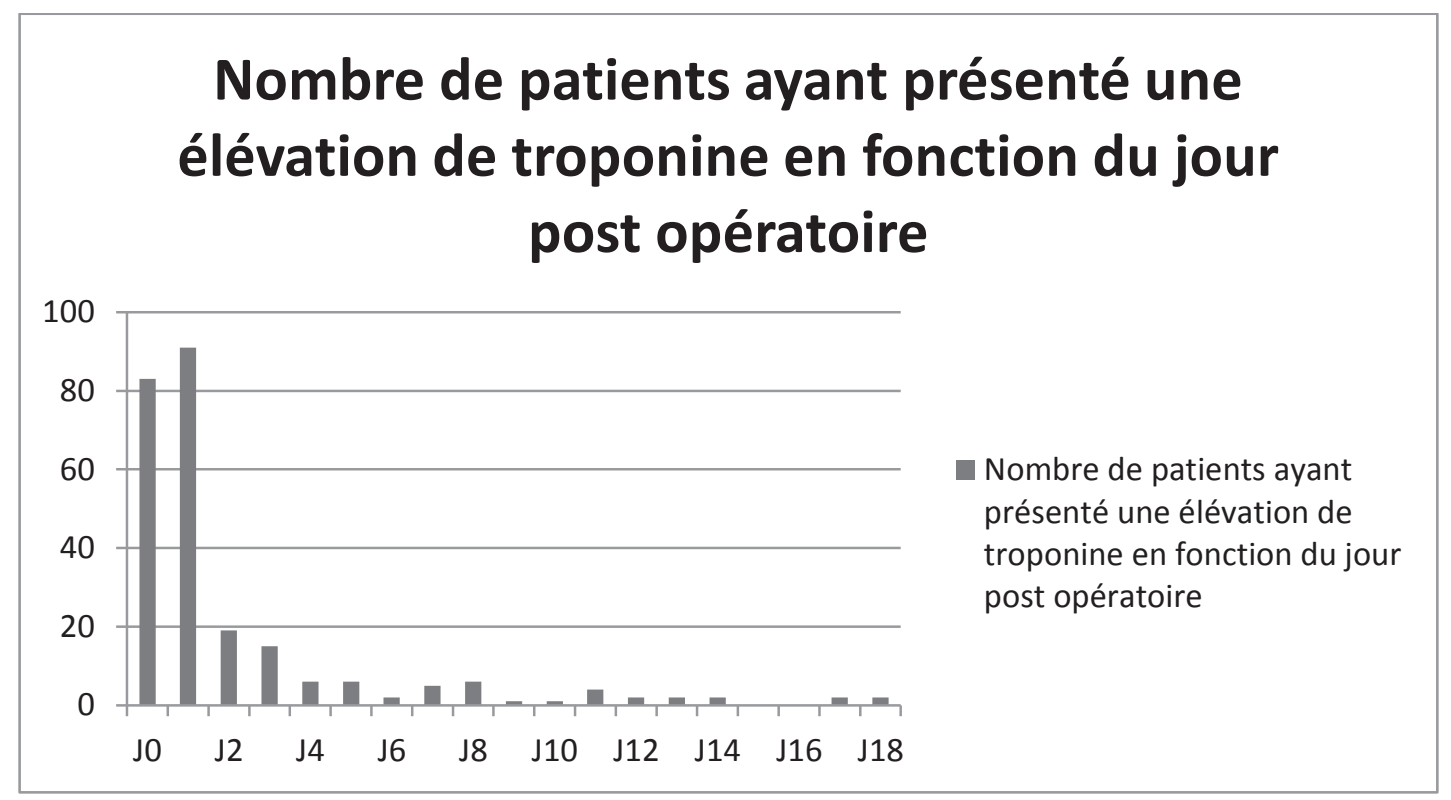

Figure 14 : nombre de patients ayant présenté une élévation de troponine en fonction du jour post opératoire 


\section{B. Patients ayant bénéficié d'une IRM cardiaque}

\section{Nombre total d'IRM cardiaque}

Parmi les 249 patients éligibles, 225 n'ont pas pu bénéficier d'une IRMC.

206 patients ont été exclus (figure 15) :

- 136 en raison d'une contre-indication ou gène à l'IRMC : 118 pour une cause rénale, 4 pour pace maker, 7 pour arythmies, 5 pour cause respiratoire, 2 pour dénutrition sévère,

- 50 ont refusé,

- 11 présentaient des troubles cognitifs, une démence ou étaient sous tutelle,

- 7 patients sont décédés avant réalisation de l'IRMC,

- 2 présentaient un patch péricardique.

Aucun patient n'a été exclu pour cause d'urgence seule. Ces patients présentaient une autre contre-indication associée : une insuffisance rénale ou un refus dans la plupart des cas.

19 autres patients n'ont pas pu bénéficier d'une IRMC en raison de difficultés organisationnelles : hospitalisation prolongée ne permettant pas la réalisation d'une IRMC, absence de coordonnées disponibles, éloignement géographique trop important.

Au total, 24 patients, 21 hommes et 3 femmes, d'âge moyen de 65 ans, ont bénéficié d'une IRM cardiaque. 


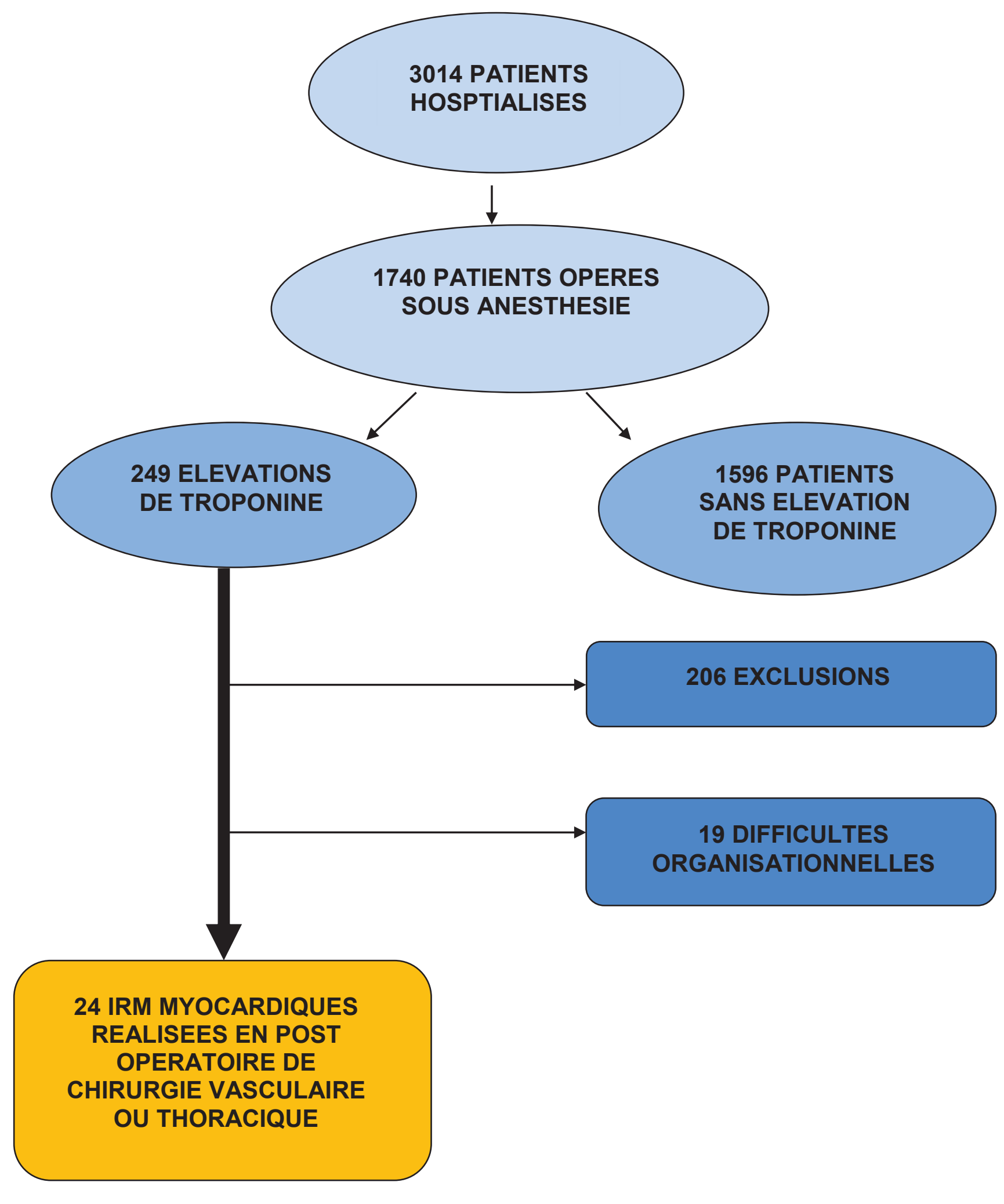

Figure 15 : schéma d'inclusion de la population 


\section{Antécédents médicaux}

Le score ASA moyen de ces 24 patients était de 3.

L'IMC moyen était de $27,1 \mathrm{~kg} / \mathrm{m}^{2}$.

Une capacité fonctionnelle supérieure à 4 MET était retrouvée chez 14 des 24 patients.

Concernant les pathologies médicales, on comptait chez ces patients (tableau I) :

- 20 patients hypertendus,

- 18 patients hypercholestérolémiques ou dyslipidémiques,

- 11 patients tabagiques actifs ou récemment sevrés (moins de 1 an),

- 8 patients diabétiques connus sous anti diabétiques oraux ou sous insuline,

- 12 patients coronariens connus dont 8 déjà revascularisés (angioplastie transluminale, stent, ou pontage).

Le Revised Cardiac Risk Index (RCRI) se répartissait ainsi : 12 patients avec un RCRI à 1, 8 RCRI à 2, 2 RCRI à 3 et 2 RCRI à 4 (figure 16).

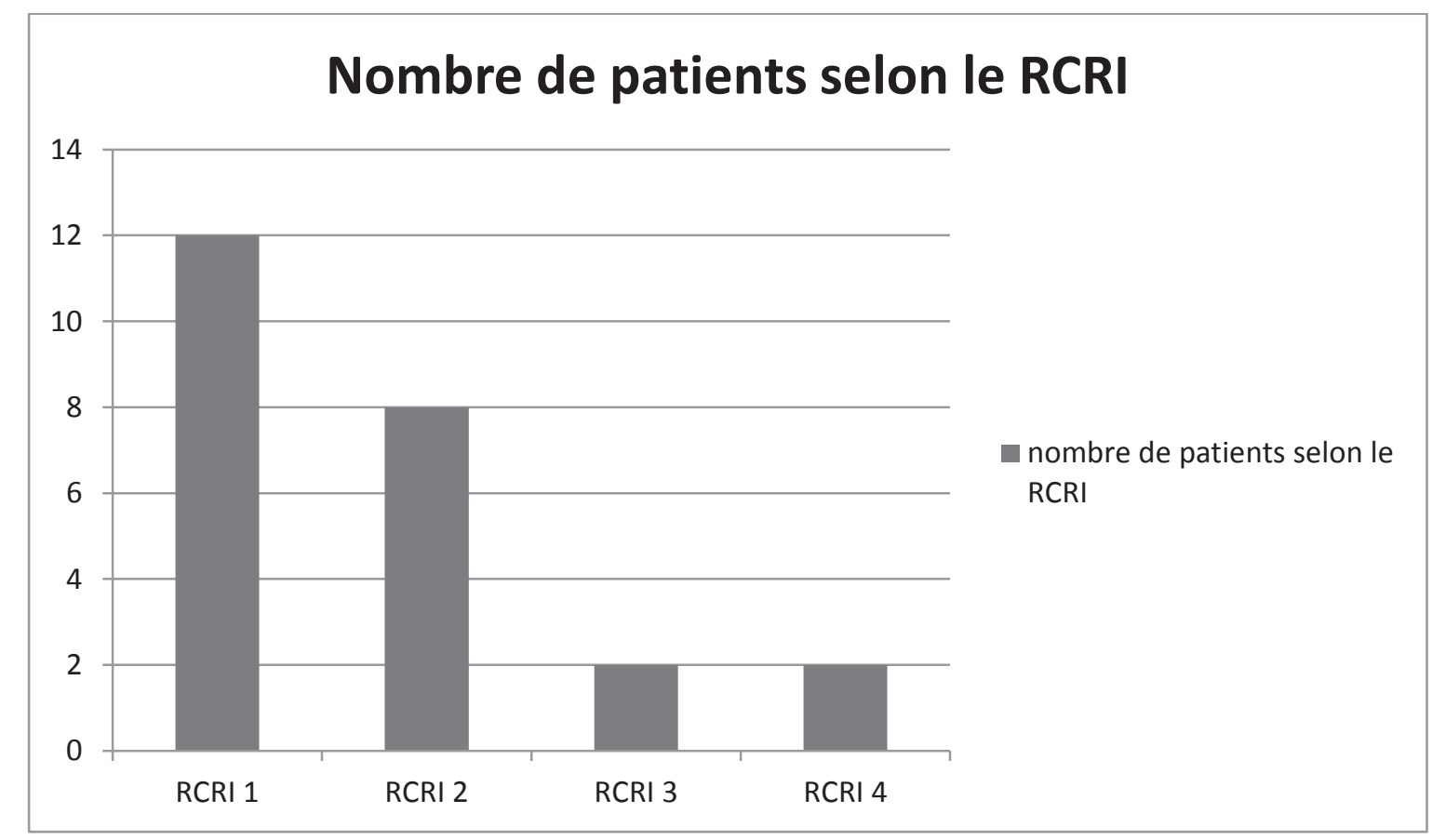

Figure 16 : nombre de patients selon le RCRI. 


\section{Traitements préopératoires}

Les traitements pré opératoires en place au moment de la consultation d'anesthésie étaient (tableau I) :

- 14 patients sous béta bloquants,

- 17 patients sous anti aggrégants plaquettaires,

- 8 patients sous inhibiteurs calciques, 14 patients sous traitement agissant sur le système rénine angiotensine,

- 17 patients sous statines.

\section{Type de chirurgie}

21 patients opérés de chirurgie vasculaire et 3 patients opérés de chirurgie thoracique programmées, tous sous anesthésie générale, ainsi répartis (tableau I) :

- 7 patients opérés de chirurgie aortique, dont 2 ayant nécessité une reprise,

- 1 reprise immédiate pour saignement massif,

- 1 reprise pour échec d'exclusion anévrysmale sur endoprothèse,

- 11 patients opérés de chirurgie vasculaire périphérique, dont 1 reprise pour échec,

- 1 patient opéré d'une chirurgie carotidienne,

- 2 amputations de membre inférieur,

- 2 lobectomies, dont 1 reprise en post opératoire immédiat pour plaie de l'artère pulmonaire,

- 1 thoracoscopie.

La durée moyenne d'intervention était de 4,5 heures. 


\begin{tabular}{|c|c|c|c|c|c|}
\hline & Critères & $\begin{array}{c}\text { Chirurgie } \\
\text { aortique } \\
\\
\mathrm{N}=7\end{array}$ & $\begin{array}{c}\text { Chirurgie } \\
\text { périphérique } \\
\text { ou } \\
\text { amputation } \\
\mathrm{N}=13\end{array}$ & $\begin{array}{l}\text { Chirurgie } \\
\text { carotidienne }\end{array}$ & $\begin{array}{c}\text { Chirurgie } \\
\text { thoracique } \\
\text { N=3 }\end{array}$ \\
\hline \multirow{6}{*}{ 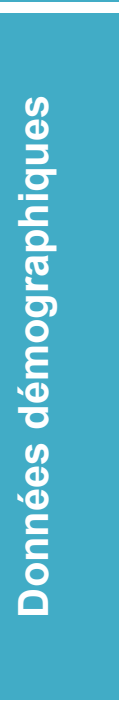 } & Hommes & 6 & 13 & 1 & 2 \\
\hline & Femmes & 1 & 0 & 0 & 1 \\
\hline & $\begin{array}{l}\text { Age moyen } \\
\text { (années) }\end{array}$ & 68 & 68 & 77 & 76 \\
\hline & $\begin{array}{r}\text { ASA } 2 \\
3 \\
4\end{array}$ & $\begin{array}{l}1 \\
5 \\
1\end{array}$ & $\begin{array}{c}0 \\
11 \\
2\end{array}$ & $\begin{array}{l}1 \\
0 \\
0\end{array}$ & $\begin{array}{l}1 \\
2 \\
0\end{array}$ \\
\hline & $\begin{array}{c}\text { IMC } \\
\left(\mathrm{kg} / \mathrm{m}^{2}\right)\end{array}$ & 25 & 28 & 26 & 27 \\
\hline & $\begin{array}{l}\text { Capacité } \\
\text { fonctionnelle } \\
\text { >4 MET } \\
\text { <4 MET }\end{array}$ & $\begin{array}{l}3 \\
4\end{array}$ & $\begin{array}{l}6 \\
7\end{array}$ & $\begin{array}{l}1 \\
0\end{array}$ & $\begin{array}{l}3 \\
0\end{array}$ \\
\hline \multirow{7}{*}{ 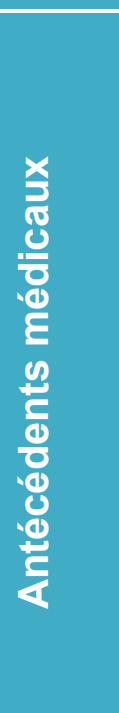 } & $\begin{array}{l}\text { Hypertension } \\
\text { artérielle }\end{array}$ & 5 & 11 & 1 & 2 \\
\hline & $\begin{array}{l}\text { Hypercholestérolém } \\
\text { ie ou dyslipidémie }\end{array}$ & 5 & 11 & 1 & 1 \\
\hline & Tabagisme actif & 3 & 7 & 0 & 1 \\
\hline & Diabète & 2 & 6 & 0 & 0 \\
\hline & $\begin{array}{l}\text { Cardiopathie } \\
\text { ischémique }\end{array}$ & 3 & 7 & 1 & 1 \\
\hline & $\begin{array}{l}\text { Antécédent de } \\
\text { revascularisation }\end{array}$ & 2 & 5 & 1 & 0 \\
\hline & $\begin{array}{r}\text { RCRI } 1 \\
2 \\
3 \\
4\end{array}$ & $\begin{array}{l}3 \\
3 \\
1 \\
0\end{array}$ & $\begin{array}{l}8 \\
2 \\
1 \\
2\end{array}$ & $\begin{array}{l}0 \\
1 \\
0 \\
0\end{array}$ & $\begin{array}{l}2 \\
1 \\
0 \\
0\end{array}$ \\
\hline \multirow{5}{*}{ 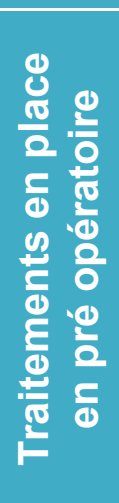 } & Béta bloquants & 3 & 9 & 1 & 1 \\
\hline & $\begin{array}{l}\text { Anti aggrégants } \\
\text { plaquettaires }\end{array}$ & 4 & 12 & 1 & 0 \\
\hline & $\begin{array}{l}\text { Inhibiteurs } \\
\text { calciques }\end{array}$ & 1 & 4 & 1 & 2 \\
\hline & $\begin{array}{l}\text { Anti hypertenseurs } \\
\text { agissant sur le } \\
\text { système rénine } \\
\text { angiotensine }\end{array}$ & 4 & 8 & 1 & 2 \\
\hline & Statines & 4 & 11 & 1 & 1 \\
\hline
\end{tabular}

Tableau I : caractéristiques démographiques et pré opératoires des 24 patients. MET : équivalents métaboliques. 


\section{Evènements per et post opératoires immédiats}

En per opératoire et post opératoire immédiat (surveillance post interventionnelle immédiate en salle de réveil), on relevait plusieurs facteurs déséquilibrant la balance en oxygène du myocarde (durée supérieure à 10 minutes) :

- Un épisode ou plus de tachycardie chez 8 patients,

- Un épisode ou plus d'hypertension artérielle chez 16 patients,

- Un épisode ou plus d'hypotension artérielle chez 13 patients,

- Une anémie chez 12 patients dont 10 ont nécessité une transfusion,

- Un épisode ou plus d'hypoxie chez 2 patients,

- Un support inotrope ou vasopresseur per opératoire a été introduit chez 5 patients : dobutamine ou noradrénaline ou association des 2.

5 patients, soit $20,8 \%$, ont présenté une modification de leur ECG entre le pré et le post opératoire :

- Un passage en arythmie cardiaque par fibrillation auriculaire paroxystique,

- Une négativation des ondes T chez 3 patients : une en latéral, une en apico-latéral et une en antéro-septal,

- Une modification électrique avec apparition d'onde $Q$ en inférieur chez un patient.

Aucun d'entre eux n'a présenté de sus-décalage du segment ST. 


\section{CINETIQUES DE TROPONINE CHEZ LES 24 PATIENTS ETUDIES}

Dans la population qui a pu être étudiée en imagerie, les 13 premiers patients avaient eu un dosage de troponine lc et les 11 suivants, un dosage de troponine $\mathrm{T}$ ultra-sensible. La valeur moyenne du pic de troponine lc était de 41,5 ng/ml (ext. : 1,12-465 $\mathrm{ng} / \mathrm{ml}$ ) et celle du pic de troponine Tus était de 125,1 $\mathrm{ng} / \mathrm{ml}$ (ext. : 19-272 $\mathrm{ng} / \mathrm{ml})$.

Le pic de troponine était atteint à J0 pour 9 patients, J1 pour 9 patients soit $79 \%$ dans les 48 premières heures, J2 pour 2 patients, aucun à J3, J4 pour 2 patients, aucun entre $\mathrm{J} 5$ et $\mathrm{J} 7$, et J8 pour 2 patients (figure 17).

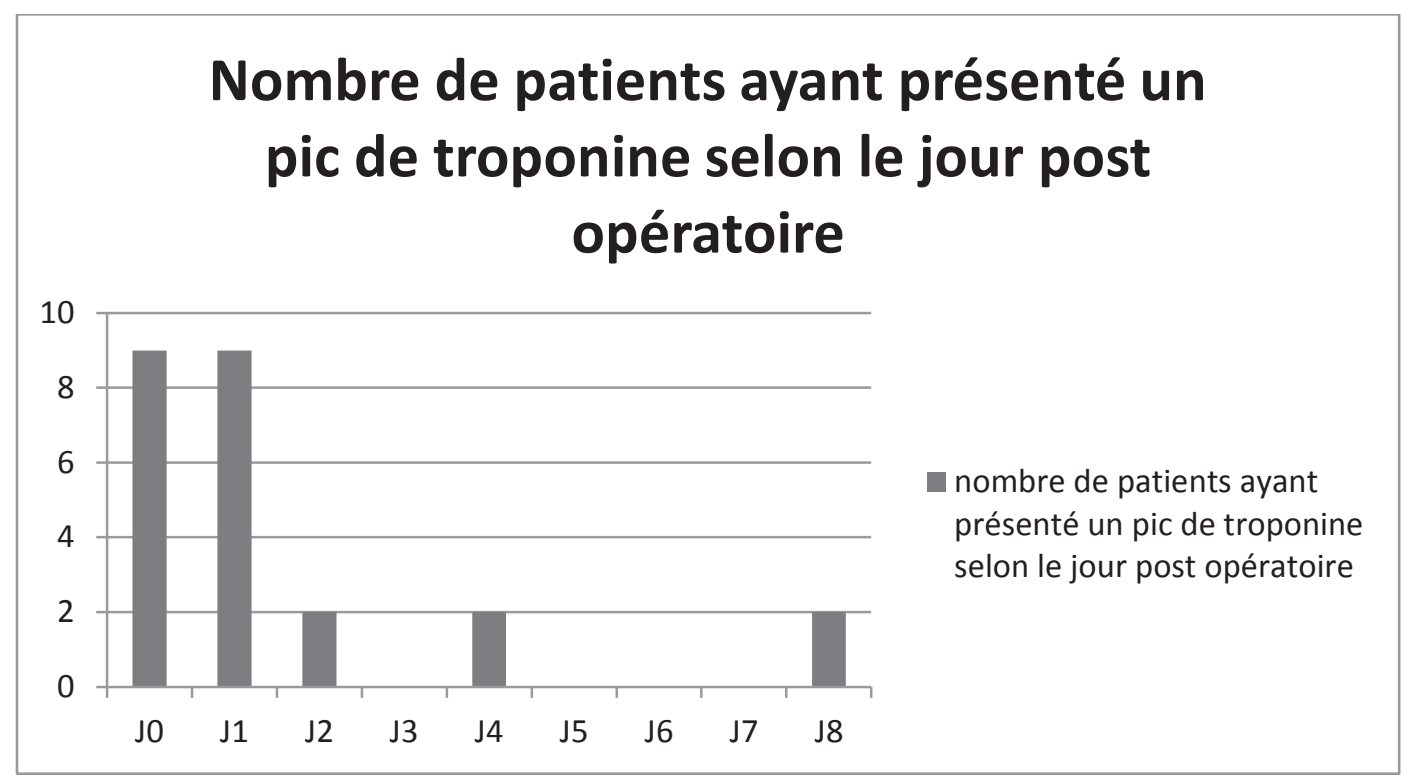

Figure 17 : nombre de patients ayant présenté un pic de troponine selon le jour post opératoire

La cinétique de troponine, en fonction du jour post opératoire, était différente selon les patients (figures 18, 19). Le nombre de patients élevant leur troponine par jour (J-1 est défini comme le jour précédent l'intervention, J0 le jour l'intervention, et J1 le jour suivant l'intervention) se répartissait comme sur la figure 20. 


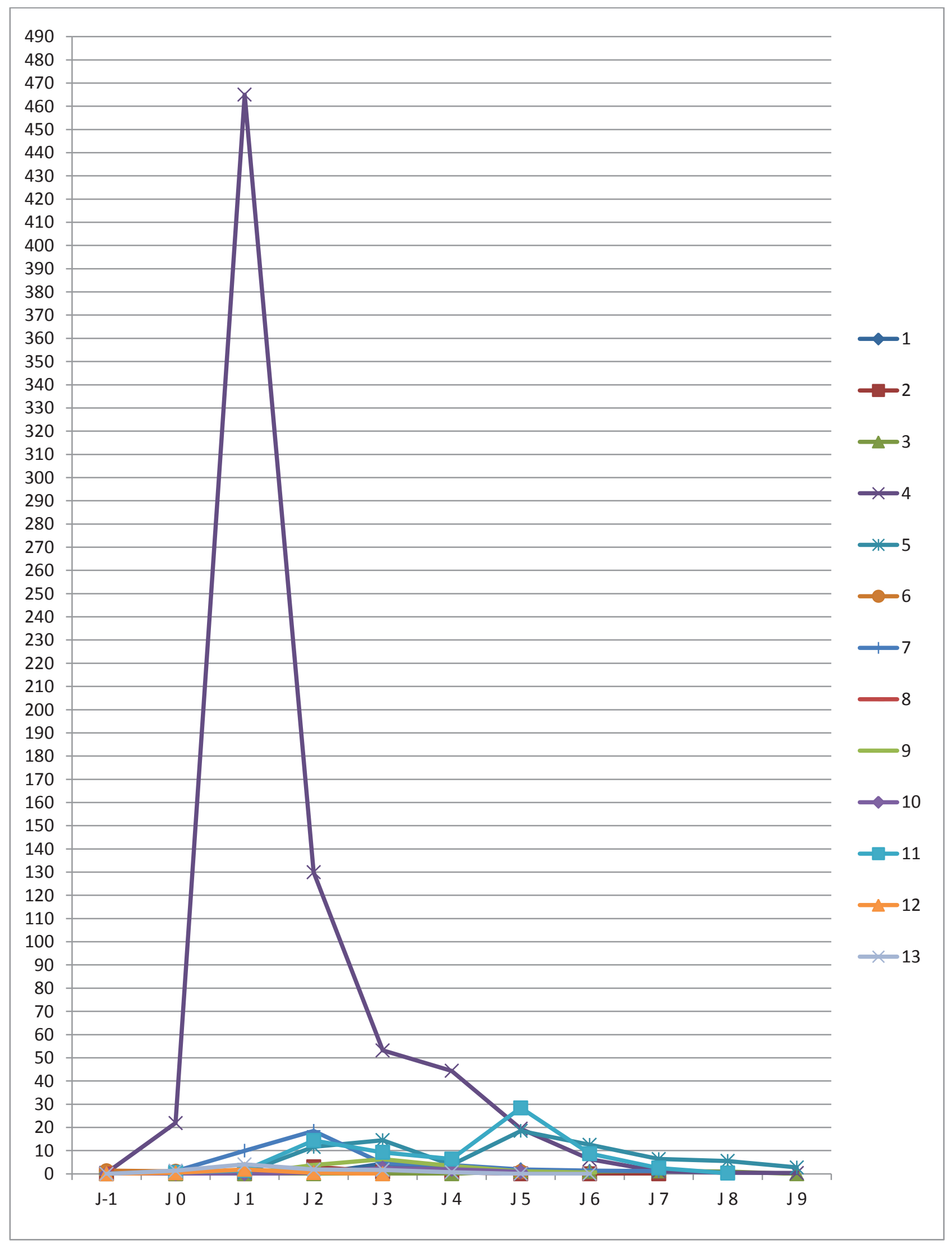

Figure 18 : cinétique de troponine Ic des 13 patients ayant eu une IRMC. 


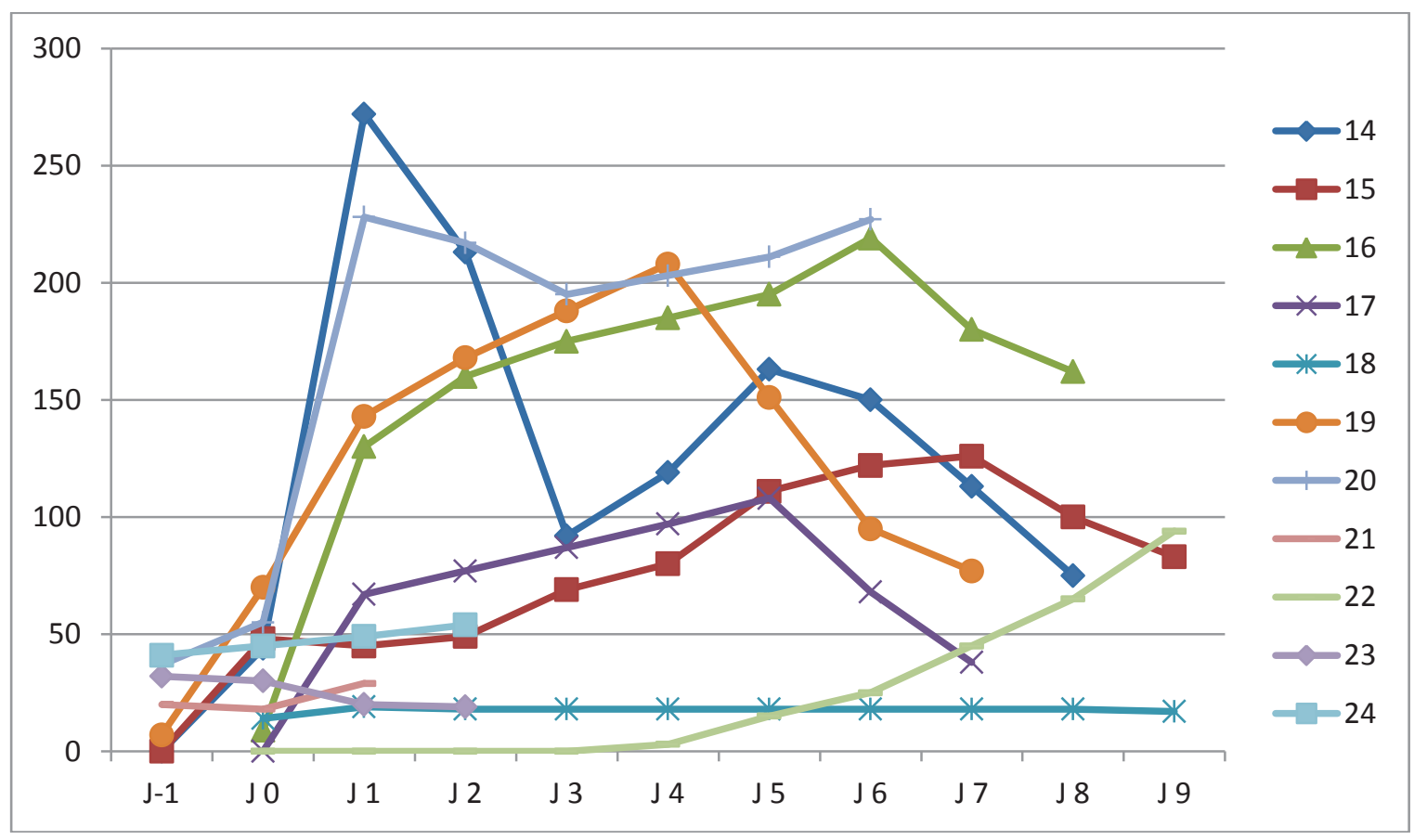

Figure 19 : cinétique de troponine T ultra-sensible des 11 patients ayant eu une IRMC

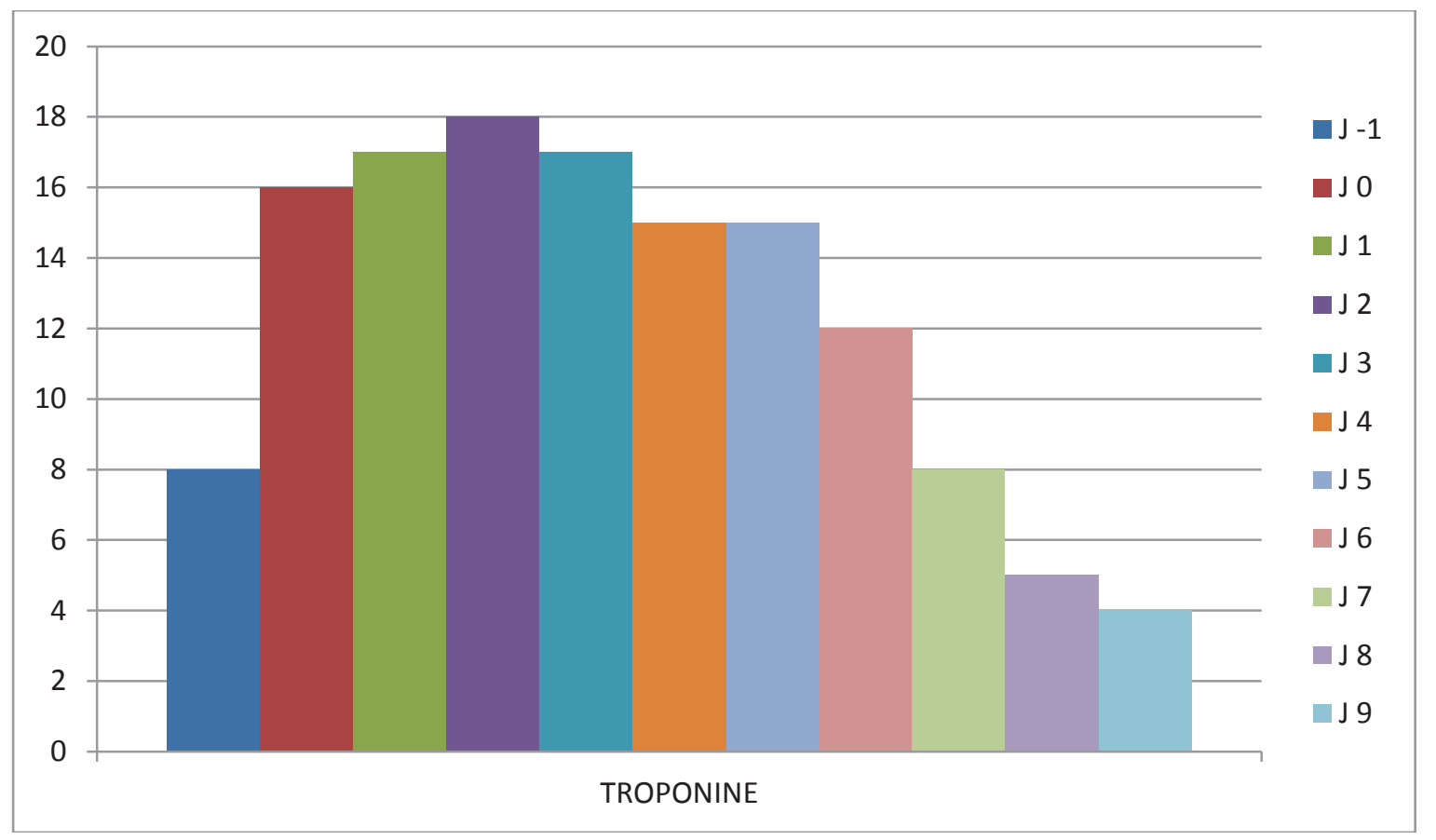

Figure 20: nombre de patients ayant une troponinémie positive par jour post opératoire, J0 étant défini comme le jour de l'intervention. 


\section{DONNEES DE L'IRM CARDIAQUE}

24 IRM cardiaques ont été réalisées durant ces 21 mois d'étude. Le délai médian de réalisation de l'IRM était de 81 jours (ext. : 4 - 380 jours).

Tous les patients ont pu bénéficier des séquences sans injection d'agent de contraste. Un seul patient n'a pu bénéficier d'injection d'agent de contraste du fait d'un faible capital veineux. Les examens étaient de bonne qualité dans l'ensemble.

\section{A. Anomalies de rehaussement tardif}

15 patients ont présenté des anomalies à I'IRM contre 9 patients sans anomalie. Les caractéristiques des patients ayant présenté une anomalie sont résumées dans le tableau II (2 pages).

\section{Transmuralité des lésions}

Chez 9 patients, les lésions étaient transmurales, soit une atteinte de $100 \%$ de l'épaisseur myocardique. Chez 6 , elles concernaient une épaisseur myocardique comprise entre 25 et $75 \%$.

\section{Lésions observées}

9 patients présentaient une lésion sur 1 ou plusieurs segments contigus. 6 patients présentaient au moins 2 localisations distinctes.

\section{Nombre de segments concernés}

Les lésions observées s'étendaient de 2 à 6 segments sur 17 segments. 


\begin{tabular}{|c|c|c|c|c|c|c|c|}
\hline & $\mathbf{N}$ & SEGMENTS & TERRITOIRE & TRANSMURALITE & FEVG & DELAI & EXPLORATIONS PRE OPERATOIRES \\
\hline \multirow{8}{*}{ 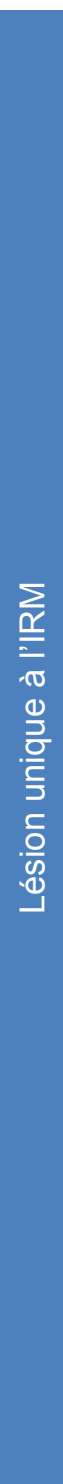 } & 1 & 4 & Inféro-latéro-basal & 50 & 61 & 91 & $\begin{array}{l}\text { Cardiopathie ischémique multi opérée. } \\
\text { Coronarographie en } 2007 \text { : sténose de la } \\
\text { circonflexe stentée puis dilatée, sténose de l'IVA } \\
\text { stentée, sténose du tronc commun stenté en } 2004 \text {. } \\
\text { ETT: FEVG } 65 \% \text {, rétrécissement aortique sans } \\
\text { HTAP. }\end{array}$ \\
\hline & 2 & 3 & $\begin{array}{l}\text { Inféro-septo-basal + } \\
\text { médian }\end{array}$ & 50 & 55 & 111 & $\begin{array}{l}\text { Coronarographie en } 2010 \text { : occlusion du segment } \\
\text { distal de la coronaire droite, sténose serrée (>70\%) } \\
\text { sur l'IVA et la Cx. } \\
\text { Scintigraphie en } 2010 \text { : séquelle inférieure sans } \\
\text { ischémie résiduelle, } 92 \% \text { FMT } \\
\text { ETT: FEVG } 68 \%\end{array}$ \\
\hline & 3 & 4 & Apico-latéral & 50 & 52 & 118 & $\begin{array}{l}\text { Cardiopathie ischémique tritronculaire stentée en } \\
2008 \text { sur la Cx proximale sur une épreuve d'effort } \\
\text { positive. } \\
\text { Epreuve d'effort négative en } 2010\end{array}$ \\
\hline & $4^{*}$ & 5 & Inférieur étendu ancien & 100 & 31 & 81 & $\begin{array}{l}\text { IDM en } 1996 \text { avec stents sur les coronaires droite et } \\
\text { I'IVA. } \\
\text { Scintigraphie en } 2010 \text { : séquelles de nécrose } \\
\text { inférieure, inféro-latérale et inféro-septale } \\
\text { concernant } 6 / 17 \text { segments sans ischémie résiduelle } \\
\text { associée. FEVG 50\%. }\end{array}$ \\
\hline & 5 & 2 & Inféro-basal & 50 & 44 & 380 & $\begin{array}{l}\text { Scintigraphie en } 2010 \text { : FEVG } 47 \% \text {, discrète } \\
\text { hypofixation septale probablement en rapport avec } \\
\text { un bloc de branche gauche, sans ischémie } \\
\text { résiduelle. } \\
\text { Triple pontage en } 1999 \text { sur l'IVA, la marginale et la } \\
\text { diagonale. }\end{array}$ \\
\hline & 6 & 1 & Inféro-basal & 100 & 46 & 46 & Epreuve d'effort en 2009 : négative à 100\% FMT \\
\hline & 7 & 3 & $\begin{array}{l}\text { Antéro } \\
\text {-médian }\end{array}$ & 100 & 50 & 38 & ETT en 2009 : FEVG 55\%. \\
\hline & 8 & 2 & Inféro-latéro-basal & 75 & 36 & 48 & $\begin{array}{l}\text { Cardiopathie ischémique avec IDM en } 2004 \text { et } 2 \\
\text { pontages ( } 1 \text { sur l'IVA, } 1 \text { sur la coronaire droite). } \\
\text { Scintigraphie en } 2008 \text { : séquelles inférieure ( } 2 / 17 \\
\text { segments) et inféro basale ( } 3 / 17 \text { segments), FEVG } \\
44 \% \text {. }\end{array}$ \\
\hline
\end{tabular}




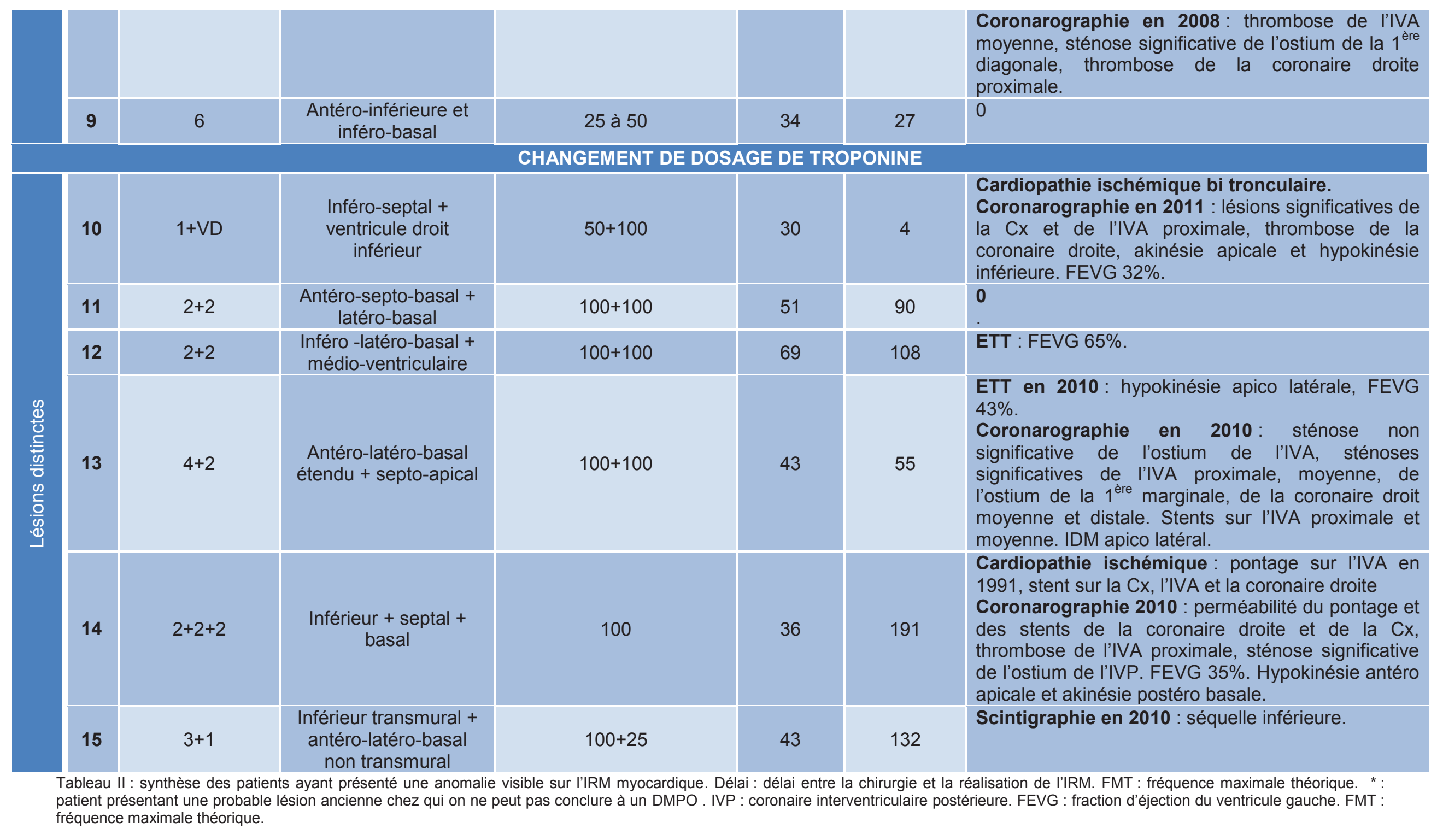




\section{B. IRMC avec une lésion unique}

9 patients présentaient une anomalie unique sur I'IRMC dont l'analyse retrouve :

- Chez le $1^{\text {er }}$ patient, l'ECG post opératoire était modifié en latéral avec des ondes $T$ négatives. L'IRMC a permis d'objectiver une lésion touchant 4 segments en inféro-latéro-basal avec une altération de la micro-circulation sur $50 \%$ de transmuralité. La coronarographie de 2007 retrouvait un bon flux coronaire au niveau des sténoses de l'artère circonflexe $(\mathrm{Cx})$, du tronc commun, et de l'artère inter ventriculaire antérieure (IVA) antérieurement revascularisées. Les modifications électriques post opératoires associées aux données de l'IRM nous font supposer qu'il s'agit d'un DMPO,

- Chez le $2^{\text {ème }}$ patient, l'IRMC retrouvait une lésion inféro-septo-basale et médiane touchant 3 segments de $50 \%$ de transmuralité. La scintigraphie et la coronarographie pré opératoires rapportaient une séquelle inférieure et une sténose significative de l'IVA et de la Cx. L'atteinte myocardique visualisée est plus étendue que celle visualisée en pré opératoire sur la scintigraphie. On peut donc supposer que les lésions individualisées par l'IRMC sont apparues en post opératoire et sont identifiables comme DMPO,

- Chez le $3^{\text {ème }}$ patient, l'ECG présentait une modification en apico-latérale avec apparition d'une nouvelle onde Q. L'IRMC retrouvait une lésion apico-latérale touchant 4 segments de $50 \%$ de transmuralité. Le dernier contrôle par coronarographie retrouvait une bonne perméabilité du stent sur la $\mathrm{Cx}$ proximale et l'épreuve d'effort était négative en pré opératoire. On peut donc supposer que la lésion non transmurale visualisée sur l'IRMC est un DMPO,

- Chez le $5^{\text {ème }}$ patient, l'IRMC individualisait une lésion inféro-basale touchant 2 segments de $50 \%$ de transmuralité, non retrouvée sur la scintigraphie pré opératoire. On peut donc supposer que la lésion est apparue en post opératoire,

- Chez le $6^{\text {ème }}$ patient, l'ECG post opératoire retrouvait une modification en inférieur. L'IRMC a individualisé une lésion inféro-basale transmurale concernant 1 segment avec un anévrysme en regard (figure 21). L'épreuve d'effort était négative en pré opératoire, à $100 \%$ de la fréquence maximale théorique (FMT). On peut donc supposer qu'il s'agit d'un DMPO, 
- Chez le $7^{\text {ème }}$ patient, on individualise une lésion de 3 segment antéro-médian transmural par IRMC. L'ETT pré opératoire ne retrouvait pas d'anomalie segmentaire. Nous supposons qu'il s'agit d'un DMPO.

- Chez le $8^{\text {ème }}$ patient, l'IRMC retrouvait une lésion inféro-latéro-basale de 2 segments de $75 \%$ de transmuralité. La coronarographie pré opératoire retrouvait une thrombose de I'IVA moyenne, une sténose significative de l'ostium de la $1^{\text {ère }}$ diagonale et une thrombose de la coronaire droite proximale. La scintigraphie pré opératoire retrouvait des séquelles inférieures touchant 2 segments et inféro-basale touchant 3 segments. Les lésions identifiées sur l'IRM sont plus étendues en latéral, laissant supposer que les séquelles individualisées en scintigraphie n'étaient pas aussi étendues que les lésions sur l'IRMC. Ceci laisse penser qu'une partie de la lésion est récemment apparue, et identifiable comme DMPO,

- Chez le $9^{\text {ème }}$ patient, on retrouvait une lésion antéro-inférieure et une autre inféro-basale s'étendant à 6 segments de 25 à $50 \%$ de transmuralité. Aucune exploration cardiologique pré opératoire n'était disponible. On suppose qu'il s'agit de 2 lésions nouvellement constituées et elles ont été identifiées comme DMPO.

Chez le $4^{\text {ème }}$ patient, l'IRMC a permis d'individualiser une lésion inférieure étendue transmurale touchant 5 segments. Cette lésion est concordante avec les données pré opératoires de scintigraphie qui retrouvait des séquelles de nécrose inférieure, inféro-latérale et inféro-septale touchant 6 segments sur 17. Cependant celle-ci ne retrouvait pas d'ischémie résiduelle associée. La lésion mise en évidence par IRMC semble donc ancienne, séquelle d'une précédente ischémie et pré existante en pré opératoire, ne permettant pas d'affirmer l'origine post opératoire. On ne peut donc pas conclure à un DMPO.

La comparaison des données de l'IRMC aux explorations cardiologiques pré et post opératoires disponibles, chez les 9 patients ayant présenté une lésion unique sur la IRMC, a permis de conclure à un DMPO chez 8 patients et à 1 lésion ancienne. 


\section{IRMC objectivant 2 lésions}

6 patients ont présenté au moins 2 anomalies distinctes (figure 22).

- Chez le $10^{\text {ème }}$ patient, une lésion inféro-septale avec $50 \%$ de transmuralité et une lésion transmurale s'étendant à toute la paroi inférieure du VD, ont été identifiées sur l'IRMC (figure 22). Les données pré opératoires retrouvaient sur la coronarographie des lésions significatives de la $\mathrm{Cx}$ et de l'IVA proximale, une thrombose de la coronaire droite, une akinésie apicale et une hypokinésie inférieure. La lésion inférieure du ventricule droit visualisée sur l'IRM peut être en rapport avec la thrombose de la coronaire droite identifiée sur la coronarographie pré opératoire. Par contre, la lésion inféro-septale non transmurale n'était pas identifiée sur les données pré opératoires. On peut supposer que cette lésion est un DMPO,

- Chez le $11^{\text {ème }}$ patient, l'IRMC retrouvait 2 lésions distinctes, la $1^{\text {ère }}$ en antérosepto-basal et la $2^{\text {nde }}$ en latéro-basal touchant 2 segments chacune et atteignant $100 \%$ de transmuralité. Aucune exploration cardiologique pré opératoire n'était disponible. Ces lésions ont été identifiées comme des DMPO,

- Chez le $12^{\text {ème }}$ patient, l'ECG post opératoire était modifié avec l'apparition d'une onde $Q$ en inférieur. On retrouvait 2 lésions transmurales touchant chacune 2 segments, la $1^{\text {ère }}$ en inféro-latéro-basal et la $2^{\text {nde }}$ en médioventriculaire sur l'IRMC. L'ETT pré opératoire ne rapportait pas de trouble de cinétique pariétale, orientant vers des lésions nouvellement constituées et vers un DMPO,

- Chez le $13^{\text {ème }}$ patient, l'ECG en post opératoire montrait un passage en AC/FA. L'IRMC individualisait 2 lésions : une $1^{\text {ère }}$ en antéro-latéro-basale de 4 segments et une $2^{\text {nde }}$ en septo-apicale touchant 2 segments. Les 2 lésions retrouvées touchaient $100 \%$ de la paroi myocardique. La coronarographie pré opératoire retrouvait une sténose non significative de l'ostium de l'IVA, des sténoses significatives de l'IVA proximale, moyenne, de l'ostium de la $1^{\text {ère }}$ marginale, de la coronaire droit moyenne et distale. 2 stents étaient présents sur l'IVA proximale et moyenne dans les suites d'un IDM apico-latéral. De plus, l'ETT retrouvait une hypokinésie dans le territoire antérieurement infarci. 
La $1^{\text {ère }}$ lésion antéro-latéro-basale peut être identifiée comme un DMPO, et la $2^{\text {nde }}$ lésion septo-apicale considérée comme ancienne,

- Chez le $14^{\text {ème }}$ patient, 3 lésions étaient retrouvées en IRMC : la $1^{\text {ère }}$ touchant 2 segments inférieurs, la $2^{\text {ème }} 2$ segments septaux et la $3^{\text {ème }}$ basale, toutes transmurales. La coronarographie pré opératoire retrouvait une perméabilité du pontage sur l'IVA et des stents de la coronaire droite et de la Cx, une thrombose de I'IVA proximale, une sténose significative de l'ostium de l'IVP. Les territoires antéro-apical et postéro-basal étaient hypokinétiques. Les lésions identifiées par IRMC ne se trouvaient pas aux sites de sténoses significatives et ne dépendent pas d'un territoire segmentaire. On peut supposer que ces lésions sont des DMPO,

- Chez le $15^{\text {ème }}$ patient, une $1^{\text {ère }}$ lésion inférieure transmurale touchant 3 segments et une $2^{\text {nde }}$ lésion antéro-latéro-basale non transmurale d'un segment, étaient individualisées en IRM. Les données pré opératoires par scintigraphie rapportaient une séquelle inférieure. On peut supposer que la $1^{\text {ère }}$ lésion visualisée sur l'IRMC était une lésion ancienne, et la $2^{\text {nde }}$ lésion antéro-latéro-basale non transmurale, nouvellement individualisée, était un DMPO.

On a conclu à au moins un DMPO chez chacun des 6 patients ayant présenté au moins 2 lésions distinctes. Chez certains patients, on visualisait la présence d'une lésion ancienne associée à un DMPO.

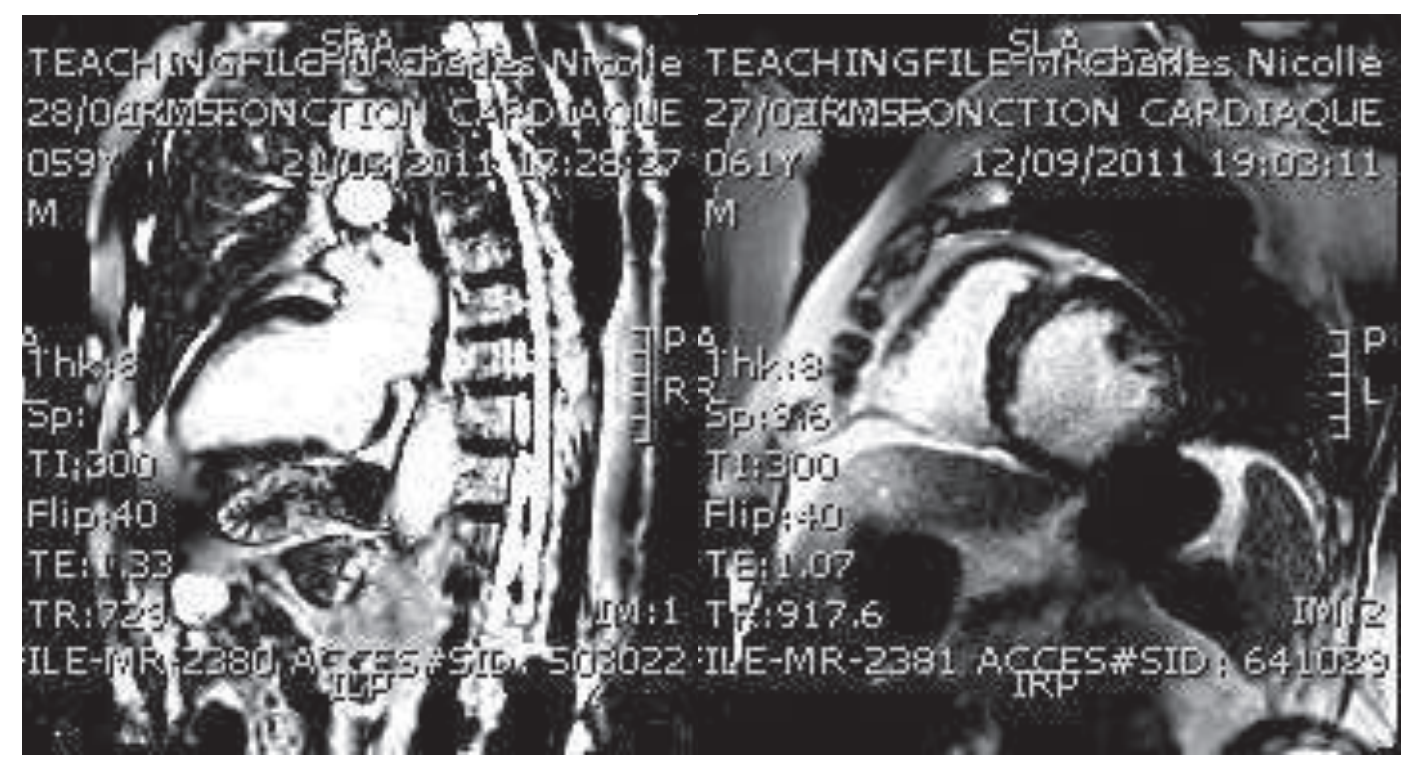

Figures 21 (à gauche) et 22 (à droite) : à gauche, DMPO inféro-basal transmural avec un anévrysme en regard, à droite, visualisation de 2 DMPO : un touchant la partie inférieure du ventricule droit et un la partie inféro-latérale du ventricule gauche 


\section{Absence d'anomalie à l'IRM cardiaque}

9 patients n'ont pas présenté d'anomalie myocardique individualisable à I'IRMC dont les résultats sont présentés dans le tableau III :

- Les $1^{\mathrm{er}}$ et $2^{\mathrm{ème}}$ patients n'avaient pas bénéficié d'exploration cardiologique pré opératoire. II n'est donc pas possible de conclure,

- Les $3^{\text {ème }}$ et $4^{\text {ème }}$ patients avaient bénéficié d'une ETT pré opératoire qui ne retrouvait pas d'anomalie de la cinétique pariétale,

- Les $5^{\text {ème }}, 6^{\text {ème }}$ et $7^{\text {ème }}$ patients avaient bénéficié d'une coronarographie pré opératoire qui retrouvait des thromboses, des sténoses significatives et non significatives dans différents territoires. Bien qu'une cardiopathie ischémique ait été mise en évidence par les explorations pré opératoires et qu'il y ait eu une élévation de troponine en post opératoire, aucune lésion n'a pu être constatée à l'IRMC,

- Le $8^{\text {ème }}$ patient avait bénéficié d'une épreuve d'effort pré opératoire négative,

- Le $9^{\text {ème }}$ patient avait bénéficié d'une scintigraphie d'effort pré opératoire négative à $80 \%$ de la FMT sans zone de séquelle mise en évidence.

Pour ces 9 patients n'ayant pas eu de lésion myocardique individualisable sur l'IRMC malgré l'élévation de troponine, et pour certains, malgré une coronaropathie ischémique documentée, une autre étiologie à l'élévation de troponine a été recherchée. Aucune donnée orientant vers une origine non ischémique n'a été retrouvée (poussée d'insuffisance cardiaque, embolie pulmonaire...).

L'analyse par IRM myocardique a permis de mettre en évidence 14 dommages myocardiques post opératoires chez les 24 patients ayant bénéficié de cet examen. Les lésions myocardiques visualisées ne sont pas segmentaires et sont indépendants des sites des sténoses significatives identifiées en pré opératoire. Ces dommages myocardiques post opératoires peuvent toucher plusieurs sites distincts, non contigus et non segmentaires. 


\begin{tabular}{|c|c|c|c|c|c|c|}
\hline $\mathbf{N}$ & SEGMENTS & TERRITOIRE & TRANSMURALITE & FEVG & DELAl & EXPLORATIONS PRE OPERATOIRES \\
\hline 1 & 0 & 0 & 0 & 68 & 49 & 0 \\
\hline 2 & 0 & 0 & 0 & 59 & 117 & 0 \\
\hline 3 & 0 & 0 & 0 & 39 & 55 & ETT 2010 : FEVG 72\%, pas de trouble de cinétique. \\
\hline 5 & 0 & 0 & 0 & 57 & 23 & $\begin{array}{l}\text { Coronarographie en } 2010 \text { : thrombose de l'IVA, } \\
\text { sténose serrée } 50 \% \text { de la Cx, FEVG 56\%, akinésie } \\
\text { apicale. } \\
\text { ETT en } 2010 \text { : FEVG } 68 \% \text {. } \\
\text { Epreuve d'effort en } 2009 \text { : sous décalage à } 93 \% \\
\text { FMT. } \\
\text { Scintigraphie } 2009 \text { : hypofixation importante } \\
\text { apicale partiellement réversible, FEVG } 48 \% \text {. }\end{array}$ \\
\hline 6 & 0 & 0 & 0 & 58 & 82 & 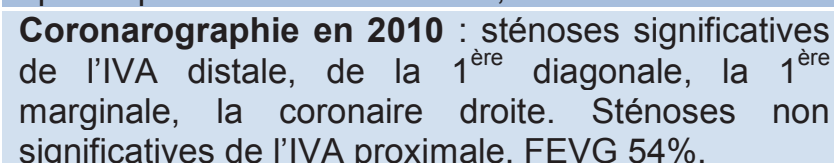 \\
\hline 7 & 0 & 0 & 0 & 57 & 48 & $\begin{array}{l}\text { Cardiopathie ischémique tri tronculaire : } 2 \\
\text { pontages en } 2005 \text { sur l'IVA et sur l'IVP, stent sur la } \\
1^{\text {ere }} \text { marginale. } \\
\text { Epreuve d'effort en } 2010 \text { : sous décalage en latéral } \\
\text { au repos et se majorant à l'effort. } \\
\text { Scintigraphie en } 2010 \text { : fixation homogène sans } \\
\text { aspect ischémique. } \\
\text { ETT en } 2010 \text { : FEVG } 50 \% \text {, sans altération de la } \\
\text { cinétique. }\end{array}$ \\
\hline$\overline{8}$ & 0 & 0 & 0 & 63 & 152 & Epreuve d'effort en 2010: négative à 91\% FMT. \\
\hline 9 & 0 & 0 & 0 & 68 & 65 & $\begin{array}{l}\text { Scintigraphie en } 2006 \text { : négative à } 80 \% \text { FMT, } \\
\text { FEVG supérieure à } 50 \% \text {. }\end{array}$ \\
\hline
\end{tabular}

Tableau III : synthèse des 9 patients n'ayant pas présenté
du ventricule gauche. FMT : fréquence maximale théorique 


\section{EVOLUTION DES PATIENTS DU GROUPE ETUDIE}

\section{A. Avis spécialisé et hospitalisation en cardiologie}

Un avis cardiologique a été demandé chez 3 des 24 patients (12,5\%) ayant eu une élévation de troponine en post opératoire de chirurgie vasculaire et thoracique. Cet avis cardiologique recommandait un suivi cardiologique en externe.

7 des 24 patients ont nécessité une hospitalisation en urgence en cardiologie dans l'année ayant suivi la chirurgie. Parmi les indications d'hospitalisation, on comptait :

- 3 décompensations cardiaques,

- 4 syndromes coronariens aigus dont 1 ayant nécessité un pontage en urgence pour infarctus du myocarde.

Un patient ( $5^{\text {ème }}$ patient du tableau III) qui ne présentait pas de lésion identifiable sur l'IRM, a nécessité une hospitalisation en cardiologie pour décompensation cardiaque.

\section{B. Taux de mortalité}

Après rappel à un an des 24 patients, on relève :

- 13 patients en vie : 11 patients ne rapportant pas de complication cardiaque à distance et 2 patients ayant été hospitalisés en service de cardiologie (revascularisation par pontage en urgence et syndrome coronarien aigu),

- 10 patients décédés,

- 1 patient perdu de vue, ne répondant pas aux appels répétés.

Le taux de mortalité à un an parmi ces patients est donc de 41,7\%.

Parmi les patients encore en vie, 9 patients présentaient une lésion sur l'IRMC contre 4 sans lésion.

Parmi les patients décédés, 4 patients présentaient une lésion sur l'IRMC contre 6 sans lésion.

Chez les patients décédés, la valeur moyenne des pics de Tnlc était de $4,5 \mathrm{ng} / \mathrm{ml}$ (ext. : $1,12-14,2 \mathrm{ng} / \mathrm{ml}$ ). 3 patients présentaient le pic de biomarqueur à J0, 1 
patient à $\mathrm{J} 1,1$ patient à $\mathrm{J} 2$ et 1 patient à $\mathrm{J} 3$. La valeur moyenne des pics de TnTus était de 183,5 ng/ml (ext. : $108-272 \mathrm{ng} / \mathrm{ml}$ ). 2 patients présentaient le pic de TnTus à J0, 1 à $\mathrm{J} 4$ et le dernier à J5. Ainsi, la majorité des patients décédés (7 patients sur 10) présentaient une élévation de troponine dans les 48 premières heures post opératoires.

Les dommages myocardiques post opératoires présentent un retentissement pronostique péjoratif important. Leur diagnostic est primordial et l'évaluation du lit micro-vasculaire est nécessaire pour la prédiction de la morbi-mortalité cardiovasculaire en post opératoire. L'IRM cardiaque semble particulièrement adaptée dans cet objectif et a permis d'identifier 14 DMPO chez 24 patients étudiés. 
DISCUSSION 


\section{CONCERNANT LA POPULATION}

\section{A. Faible nombre de patients ayant bénéficié d'une IRMC par rapport au nombre de patients éligibles}

Le nombre de patients ayant bénéficié d'une IRM cardiaque est faible en comparaison aux 249 patients éligibles soit 9,6\%.

En effet, la principale cause d'exclusion était une insuffisance rénale $(57,2 \%$ des 206 patients exclus). L'insuffisance rénale a une grande prévalence dans cette population chirurgicale, notamment du fait du poids des antécédents (athérosclérose, tabagisme, hypercholestérolémie) et de l'âge avancé. Du fait du risque de fibrose néphrogénique, le seuil de clairance a été fixé à $60 \mathrm{ml} / \mathrm{mn}$ limitant ainsi le nombre de patients inclus.

\section{B. Patients ayant refusé l'examen}

L'autre cause majeure d'exclusion a été le refus de l'examen (50 patients), malgré les explications données par téléphone sur les tenants et les aboutissants de l'examen ainsi que ses modalités. La raison principale invoquée était la peur relative à l'inconnu de la technique d'IRM, et ce malgré les explications données sur le caractère non irradiant et non invasif de la technique. De même, les patients suivis pour néoplasie pulmonaire ou ayant eu des suites post opératoires compliquées, avaient un mauvais vécu de leur hospitalisation précédente et peur d'une récidive de complication, contribuant ainsi à la limitation du nombre d'inclusions.

\section{Perdus de vue et difficultés organisationnelles}

On comptait également 19 patients exclus pour d'autres raisons : certains n'avaient pas communiqué leurs coordonnées, d'autres avaient eu des suites post opératoires compliquées nécessitant une hospitalisation prolongée et limitant leur déplacement.

Tous ces facteurs ont limité le nombre de patients candidats à une caractérisation des DMPO par IRMC. 


\section{CONCERNANT LE DOSAGE DE TROPONINE}

\section{A. Incidence d'élévation de troponine}

L'incidence d'élévation de troponine en post opératoire de chirurgie vasculaire et thoracique était de $14,3 \%$ et la grande majorité des patients a présenté le pic de troponine le jour même ou le jour suivant l'intervention. Ces résultats sont similaires aux observations présentes dans la littérature ${ }^{4,10,14,34}$.

Ceci confirme que les patients de chirurgie vasculaire et thoracique doivent bénéficier d'une vigilance importante sur le plan cardiologique, d'autant plus que les élévations de troponine post opératoires sont associées à une augmentation de la mortalité, proportionnellement au pic du marqueur.

\section{B. Evolution vers une troponine ultra-sensible}

Dans le courant du mois de mars 2011, l'hôpital Charles Nicolle a changé de type de troponine. Le laboratoire est passé d'une troponine standard Ic dont le seuil de positivité du $99^{\text {ème }}$ percentile était fixé à $1 \mathrm{ng} / \mathrm{ml}$, à une troponine de nouvelle génération ultra-sensible. Cette nouvelle norme de troponine $T$ ultra-sensible présentait un seuil de positivité du $99^{\mathrm{ème}}$ percentile à $14 \mathrm{ng} / \mathrm{ml}$.

Les formes ultra-sensibles permettent une détection et une prise en charge plus précoces des dommages myocardiques post opératoires ${ }^{72}$. En conséquent, l'incidence d'élévation de troponine a donc augmenté avec le changement de type de dosage, faisant passer une incidence d'augmentation du biomarqueur de $4,2 \%$ à $26,7 \%$. Cette augmentation de sensibilité des formes ultra-sensibles s'accompagne d'une diminution parallèle de la spécificité ${ }^{40-42,73,72}$.

II est intéressant de noter que les 9 patients dont I'IRM est négative présentaient une élévation de troponine aussi bien standard qu'ultra-sensible. 


\section{ANALYSE DES IRM CARDIAQUES}

\section{A. Localisation des dommages myocardiques post opératoires}

Des dommages myocardiques post opératoires ont pu être identifiés chez 14 des 24 patients ayant présenté une élévation de troponine en post opératoire de chirurgie vasculaire et thoracique et ayant bénéficié d'une IRMC. Cet examen a permis de visualiser directement les lésions myocardiques associées aux élévations de troponine en post opératoire. Les DMPO chez ces 14 patients sont non segmentaires et étendus en moyenne à 2 segments. Ils peuvent toucher une zone unique ou plusieurs zones non contiguës du myocarde. Ces DMPO sont indépendants des zones de sténoses significatives présentes en pré opératoire, confirmant ainsi les constatations dans les études précédentes ${ }^{4,} 17$.

La prédictivité du territoire myocardique lésé en post opératoire est difficilement prévisible du fait du réseau de collatéralité et de la micro-vascularisation. L'équipe de Poldermans et al. $^{74}$ s'était intéressée à la valeur prédictive de l'échocardiographie de stress pré opératoire en termes de territoire myocardique infarci après chirurgie vasculaire majeure chez 1065 patients. Les résultats échographiques étaient alors comparés aux résultats des autopsies réalisées chez les patients décédés dans les 30 jours post opératoires. Sur les $53 \%$ de patients décédés de cause cardiaque, l'échographie s'était révélée positive chez $50 \%$ des patients avec au moins un territoire positif en pré opératoire. Chez $50 \%$ des patients, I'IMPO s'étendait au-delà du territoire ischémique identifié par échographie. Cette sous-estimation du territoire myocardique serait due à la variabilité du réseau de suppléance coronarien confirmant ainsi l'importance de la micro-vascularisation sous-jacente. Les résultats de notre étude sont en accord avec ces résultats. II n'a pas été possible, d'après les explorations cardiologiques pré et post opératoires, de prédire les sites de DMPO.

En revanche, la majorité des patients chez qui un DMPO a été identifié, présentaient une coronaropathie connue. On retrouve des résultats similaires dans la littérature. En effet, il a été observé qu'un taux de sténoses supérieur à $30 \%$ sur l'ensemble du réseau coronaire est un facteur indépendant de complications cardiovasculaires, notamment de développer un IMPO ${ }^{17}$. 


\section{B. Altérations de la micro-circulation}

L'IRM cardiaque avec rehaussement tardif est l'examen de référence dans l'exploration de la micro-circulation myocardique. Dans une étude expérimentale de Wu et al. ${ }^{75}$, les auteurs avaient évalué, par IRMC précoce, la zone d'obstruction micro-vasculaire myocardique chez des chiens après des infarctus médicaux expérimentaux. Ils constataient que la zone d'obstruction micro-vasculaire après reperfusion était plus large que la zone infarcie et proposaient d'agir sur cette microvascularisation pour limiter l'extension de l'infarctus.

Chez les patients de notre étude, cette altération du lit micro-vasculaire a également été mise en évidence. Ces zones de fragilité micro-circulatoire au niveau de la paroi myocardique font le lit de futurs infarctus transmuraux ${ }^{5,10,11 .}$

\section{Comparaison des données de l'IRMC aux données cardiologiques pré et post opératoires disponibles}

Le caractère nouvellement constitué de ces lésions myocardiques a été établi en confrontant les explorations cardiaques pré et post opératoires disponibles, aux données de l'IRMC.

Les séquences de rehaussement tardif sur les IRMC permettant d'objectiver les lésions fibreuses cicatricielles secondaires aux ischémies. On ne peut cependant pas dater ces lésions.

Aucune séquence $T 2$, à la recherche d'œdème péri-lésionnel signant une lésion ischémique aigue récente ${ }^{76},{ }^{77}$, n'a été réalisée lors de l'étude. En effet, l'étude ne s'intéressait pas aux lésions aigues mais aux dommages myocardiques fixés en post opératoire.

L'IRMC n'étant pas un examen recommandé en pré opératoire, une comparaison pré/post opératoire n'a donc pas pu être possible. 


\section{Anomalies à l'IRM cardiaque}

Le délai médian entre la chirurgie et l'IRMC était de 81,5 jours.

Dans des études expérimentales animales de la littérature, on retrouve une fixation des altérations micro-circulatoires dans un délai de 8 semaines après revascularisation, définissant ainsi la taille définitive et la transmuralité de la lésion ischémique finale $^{75,78}$ (figure 23).

De même, chez l'Homme, dans l'étude de Kim et al. ${ }^{79}$, les auteurs ont retrouvé une corrélation entre le pourcentage de transmuralité sur l'IRMC avec rehaussement tardif, réalisée dans un délai moyen de 14 jours, et la viabilité myocardique. Dans les zones infarcies avec une atteinte transmurale du myocarde inférieure à $25 \%$, les lésions étaient de bon pronostic de récupération. Par contre, les lésions atteignant plus de $75 \%$ de transmuralité n'étaient pas viables. Entre 25 et $75 \%$, le pronostic était incertain et représentait la zone de dysfonction microcirculatoire encore non fixée.

La majorité des IRM de notre étude a été réalisée au-delà de ce délai. Ceci peut représenter un biais, expliquant que l'on ne visualise que les DMPO les plus importants. En effet, plus de la moitié des IRMC qui mettent en évidence une lésion retrouvent des DMPO atteignant 75 à 100\% de transmuralité. Par ailleurs, on rappelle que 9 patients n'ont pas présenté de lésion myocardique identifiable sur les séquences tardives de l'IRM. Pourtant, certains de ces patients présentaient une cardiopathie ischémique évoluée avec des sites de sténose significative. Chez ces patients, I'IRM a été réalisée tardivement, au-delà du délai de 8 semaines. Ce délai de réalisation de l'examen peut expliquer une partie des résultats négatifs, puisque l'on sait que les lésions myocardiques viables à potentiel de récupération ne sont plus visualisées au-delà de plusieurs semaines.

Des IRMC plus précoces auraient peut-être pu mettre en évidence des lésions ischémiques. Ce problème de délai s'intègre dans un problème plus global d'organisation et de faisabilité de cet examen en pratique courante. 


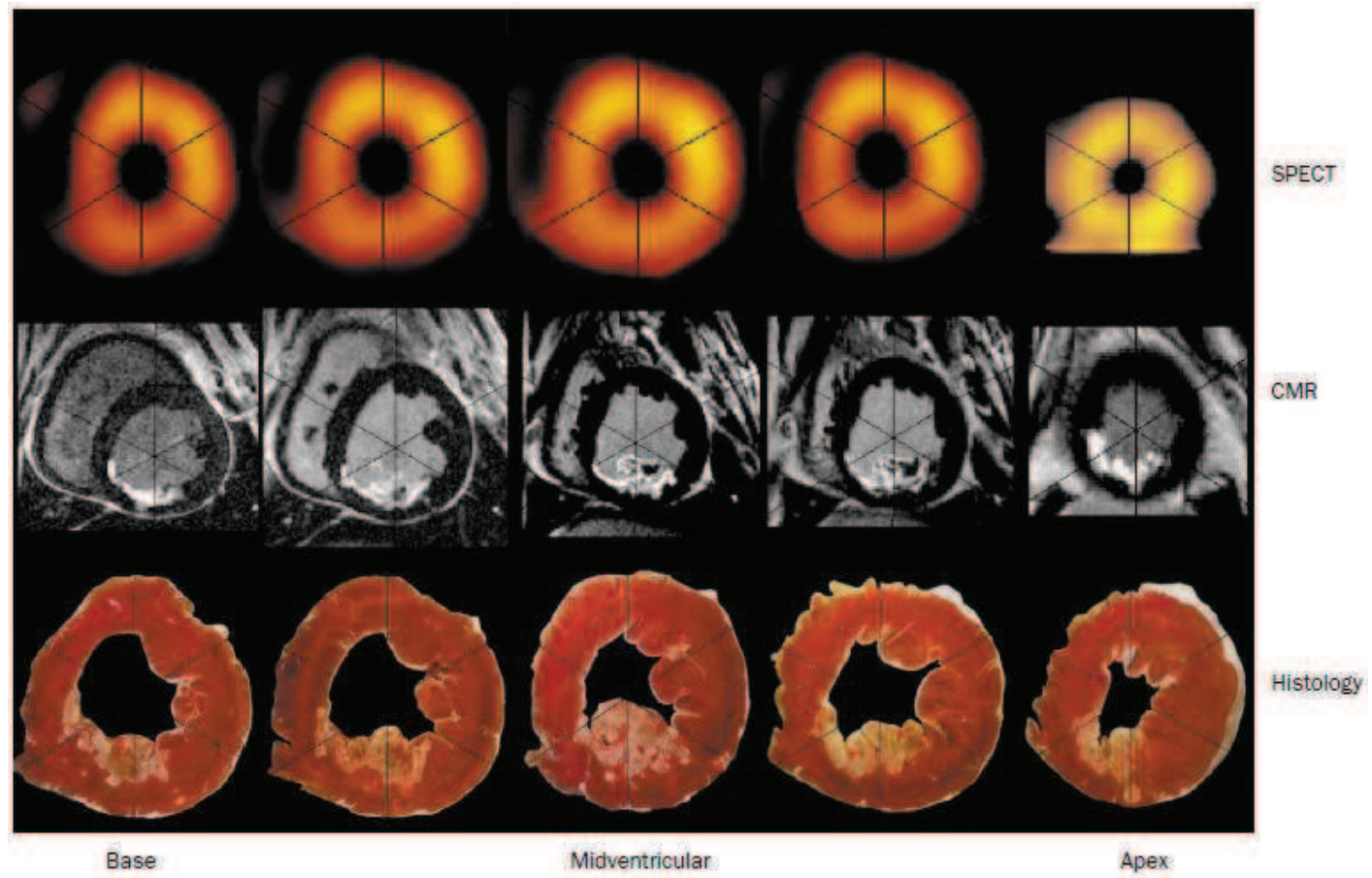

Figure 1: Typical animal SPECT and CMR images, and histological slides

Figure 23: comparaison des images d'infarctus du myocarde canin en tomoscintigraphie myocardique (SPECT), d'IRM cardiaque (CMR) et de coupes histologiques, d'après Wagner et al. $2003^{78}$ 


\section{PRONOSTIC A 1 AN DES DOMMAGES MYOCARDIQUES POST OPERATOIRES}

\section{A. Taux de mortalité}

Les 24 patients ayant bénéficié d'une IRM cardiaque ont été rappelés 1 an après la chirurgie. $41,7 \%$ des patients étaient décédés à ce terme. Parmi les patients décédés, 4 patients présentaient un DMPO identifié sur l'IRM et 6 n'en présentaient pas.

Que ces lésions soient identifiées visuellement par imagerie ou non, une élévation de troponine est associée à un pronostic péjoratif. Cette constatation est corroborée aux données de la littérature, qu'il s'agisse de troponine Ic $^{12,26-28}$ ou de troponine T ultra-sensible $e^{28}$.

Le taux de mortalité de $41,7 \%$ chez les patients de l'étude est très élevé. Une étude récente de $2012^{28}$ s'est intéressée à la corrélation entre pic de troponine ultrasensible et mortalité à 2 ans et demi. La mortalité augmentait de manière parallèle au pic de troponine : un pic inférieur à $0,03 \mu \mathrm{g} / \mathrm{l}$ était associé à une mortalité de $6 \%$, un pic entre 0,03 et $0,09 \mu \mathrm{g} / \mathrm{l}$ était associé à une mortalité de $17 \%$, et un pic supérieur à $0,09 \mu \mathrm{g} / \mathrm{l}$ était associé à une mortalité de $46 \%$. Dans notre étude, les pics de troponine ultra-sensible étaient tous au-dessus de cette valeur chez les patients décédés $(126,272,228$ et $108 \mathrm{ng} / \mathrm{ml})$.

De même, les patients décédés à 1 an post opératoire avaient présenté le pic de biomarqueur dans les 5 premiers jours suivant l'intervention. Dans l'étude de Le Manach et al. ${ }^{10}$, les auteurs observaient des résultats similaires avec une mortalité plus importante dans la population élevant sa troponine précocément. Des résultats comparables ont été retrouvés dans les études post mortem décrites par Biccard et al. ${ }^{4}$, où le taux de décès était plus important chez ces patients dont l'élévation du biomarqueur survenait dans les premiers jours post opératoires. 


\section{B. Prise en charge post opératoire immédiate}

Aucune prise en charge standardisée n'a été établie en post opératoire. Une faible proportion des patients a bénéficié d'un avis cardiologique. Cet avis spécialisé recommandait une réévaluation et un suivi en externe. A ce jour, il n'y a pas de prise en charge standardisée au sein du service de chirurgie vasculaire et thoracique du $\mathrm{CHU}$ de Rouen, en cas d'élévation de troponine en post opératoire. Or il s'agit le plus fréquemment d'augmentations isolées en post opératoire. La création et l'évaluation d'un protocole serait une voie de recherche afin d'améliorer la prise en charge cardiologique de ces patients et leur pronostic à court et à long terme. Au sein des hospices civils de Lyon, une fiche de liaison avec le service de cardiologie a été créée permettant ainsi de stratifier ces patients selon leur niveau de risque et de protocoliser leur prise en charge post opératoire ${ }^{5}$ (Annexe 3 : figures 29, 30, 31).

Un protocole de ce type serait à développer au sein du service après discussion entre cardiologues, chirurgiens, radiologues et anesthésistes. 
CONCLUSION 
Dans cette étude monocentrique prospective observationnelle menée durant 21 mois au $\mathrm{CHU}$ de Rouen au sein du service de chirurgie vasculaire et thoracique, nous avons pu mettre en évidence des dommages myocardiques post opératoires par IRM cardiaque chez 14 des 24 patients qui élèvent leur troponine en post opératoire.

De réelles lésions anatomiques ont été objectivées grâce aux IRMC réalisées. Toutes ne sont pas en lien avec des sténoses coronariennes significatives, confortant une physiopathologie des DMPO basée sur un déséquilibre de la balance en oxygène avec altération de la micro-circulation de la paroi myocardique.

Par ailleurs, dans plus de la moitié des IRMC pathologiques, les lésions identifiées sont transmurales et définitives.

D'autre part, on constate sur cette série de 24 patients une mortalité à 1 an très élevée. Ainsi, du fait du retentissement pronostique et fonctionnel d'une élévation de troponine en post opératoire de chirurgie vasculaire et thoracique, il est nécessaire d'organiser une prise en charge adaptée et ciblée afin d'optimiser le devenir de ces patients.

Une réflexion centrée sur une prise en charge pluri disciplinaire réunissant anesthésistes, chirurgiens, cardiologues et radiologues, apparait indispensable. 


\section{REFERENCES BIBLIOGRAPHIQUES}

\footnotetext{
${ }^{1}$ Mangano DT. Perioperative cardiac morbidity. Anesthesiology $1990 ; 72: 153$ - 184

2 Thygesen K, Alpert JS, White HD, joint European Society of Cardiology (ESC)/ American College of Cardiology Foundation (ACCF)/ American Heart Association (AHA)/ World Heart Federation (WHF) task force for the redefinition of myocardial
} infarction. Universal definition of myocardial infarction. Expert consensus document. Circulation 2007 ; 50 (22) : 2173 - 2195

${ }^{3}$ Piriou V. Questions fréquemment posées : surveillance post opératoire du coronarien. http://www.sfar.org/_docs/articles/107-SPO_coronarien.pdf in [www.sfar.org] (consulté le 24 août 2012)

${ }^{4}$ Biccard BM, Rodseth RN. Pathophysiology of peri-operative myocardial infarction. Anaesthesia 2010 jul ; 733 - 741

${ }^{5}$ Piriou V, Bonnefoy-Cudraz E. Surveillance des complications cardiaques post opératoires. $52^{\text {ème }}$ congrès national d'anesthésie et de réanimation. Médecins. Conférence d'actualisation de la Société Française d'Anesthésie et de Réanimation 2010. www.sfar.org (consulté le 14/06/2012)

${ }^{6}$ Siniorakis E, Kirvassilis G, Exadactylos N. Intraoperative myocardial infarction in non cardiac surgery : is a universal definition feasible ? Eur Heart J. 2008 ; 29 : 567

${ }^{7}$ Hafez HM, Berwanger CS, McColl A, Richmond W, Wolfe JHN, Mansfield AO, Stansby G. Myocardial injury in major aortic surgery. J Vasc Surg 2000 Apr ; 31 (4) : $742-750$

${ }^{8}$ Master AM, Dack S, Jaffe HJ. Postoperative coronary artery occlusion. JAMA 1938 ; $110(18): 1415-1418$

${ }^{9}$ Girard C, Meyer M, Grosjean S, Piriou V. Biomarqueurs cardiaques péri opératoires. $51^{\text {ème }}$ congrès national d'anesthésie et de réanimation. Médecins. Conférences d'actualisation. 2009 Elsevier Masson. 
${ }^{10}$ Le Manach Y, Perel A, Coriat P, Godet G, Bertrand M, Riou B. Early and delayed myocardial infarction after abdominal aortic surgery. Anesthesiology 2005 May ; 102 (5) : $885-891$

${ }^{11}$ Coriat $\mathrm{P}$, Amour J. Mécanisme et prévention des complications coronariennes de la chirurgie non cardiaque. Les essentiels 2006 ; p 253 - 263

12 Godet G, Dumerat M, Baillard C, Ben Ayed S, Bernard MA, Bertrand M, Kieffer E, Coriat P. Cardiac troponin I is reliable with immediate but not medium term cardiac complications after abdominal aortic repair. Acta Anaesthesiol Scand. 2000 ; 44 (5) : $592-597$

${ }^{13}$ Bursi F, Babuin L, Barbieri A, Politi L, Zennaro M, Grimaldi T, Rumolo A, Gargiulo M, Stella A, Modena MG, Jaffe AS. Vascular surgery patients : peri operative and long term risk according to the ACC/AHA guidelines, the additive role of post operative troponin elevation. Eur Heart J. 2005 Nov ; 26(22) : 2448 - 2456

${ }^{14}$ Landesberg G, Mosseri M, Zahger D, Wolf Y, Perouansky M, Anner H, Drenger B, Hasin Y, Berlatzky Y, Weissman C. Myocardial infarction after vascular surgery : the role of prolonged stress-induced, ST depression-type ischemia. J Am Coll Cardiol. 2001 Jun ; $37: 1829-1845$

${ }^{15}$ Poldermans D, Bax JJ, Boersma E, De Hert S, Eeckhout E, Fowkes G, Gorenek B, Hennerici MG, Lung B, Kelm M, Per Kjeldsen K, Kristensen SD, Lopez-Sendon J, Pelosi P, Philippe F, Pierard L, Ponikowski P, Schmid JP, Sellevold OFM, Sicari R, Van den Berghe G, Vermassen F, Hoeks SE, Vanhorebeek I. Guidelines for pre operative cardiac risk assessment and peri operative cardiac management in non cardiac surgery of the European Society of Cardiology (ESC) and endorsed by the European society of Anaesthesiology. Eur Heart J. 2009 ; 30(22) : 2768 - 2812

${ }^{16}$ Priebe HJ. Pre operative cardiac management of the patient for non cardiac surgery : an individualized and evidence based approach. Br J Anaesth. 2011 Jul ; $107(1): 83-96$

17 Société française d'anesthésie et de réanimation (SFAR), Société française de cardiologie (SFC). Prise en charge du coronarien qui doit être opéré en chirurgie non 
cardiaque : recommandations formalisées d'experts SFAR/SFC 2010. www.sfar.org (consulté le 24/08/2012)

${ }^{18}$ Flu WJ, Schouten O, van Kuijk JP, Poldermans D. Perioperative damage in vascular surgery patients. Eur J Vasc Endovasc Surg. 2010 Jul ; 40(1) : 1 - 8

${ }^{19}$ Brown OW, Hollier LH, Pairolero PC, Kazmier FJ, McCready RA. Abdominal aortic aneurysm and coronary artery disease. Arch Surg. 1981 ; 116 (11) : 1484 - 1488

${ }^{20}$ Guerre-Berthelot P, Crama P, Prima F, Oddoze C, Branchereau A, Gouin F, et al. Incidence des lésions myocardiques après chirurgie vasculaire : diagnostic par la troponine Ic. Ann Fr Anesth Réanim. 1997 ; 16(8) : 950 - 4

${ }^{21}$ Feringa HHH, Bax JJ, Boersma E, Dertai MD, Meij SH, Galal W, Schouten O, Thomson IR, Klootwijk P, van Sambeek MRHM, Klein J, Poldermans D. High dose $\beta$ blockers and tight heart rate control reduce myocardial ischemia and troponin $\mathrm{T}$ release in vascular surgery patients. Circulation. 2006 ; 114 (SI) : $344-349$

${ }^{22}$ Bolliger D, Seeberger MD, Lurati Buse GA, Christen P, Rupinski B, Gürke L, Filipovic M. A premiminary report on the prognostic significance of preoperative brain natriuretic peptide and postoperative troponin in patients undergoing major vascular surgery. Anesth Analg. 2009 Apr ; 108(4) : 1069 - 75

${ }^{23}$ Redfern G, Rodseth RN, Biccard BM. Outcomes in vascular surgical patients with isolated post operative troponin leak: a meta analysis. Anesthesia. 2011 ; 66 : 604 610

${ }^{24}$ Vascular Events In Noncardiac Surgery Patients Cohort Evaluation (VISION) Study Investigatiors, Deveraux PJ, Chan MT, Alonso-Coello P, Walsh M, Berwanger O, Villar JC, Wang CY, Garutti RI, Jacka MJ, Sigamani A, Srinathan S, Biccard BM, Chow CK, Abraham V, Tiboni M, Pettit S, Szcseklik W, Lurati Buse G, Botto F, Guyatt G, Heels-Ansdell D, Sessler DK, Thorlund K, Garg AX, Mrkobrada M, Thomas S, Rodseth RN, Pearse RM, Thabane L, McQueen MJ, Van Helder T, Bhandari M, Bosch J, Kurz A, Polanczyk C, Malaga G, Nagele P, Le Manach Y, Leuwer M, Yusuf S. Association between postoperative troponin levels and 30-day mortality among patients undergoing non cardiac surgery. JAMA. 2012 Jun 6 ; 307(21) : 2295 - 304 
${ }^{25}$ Bursi F, Babuin L, Barbieri A, Politi L, Zennaro M, Grimaldi T, Rumolo A, Garguilo M, Stella A, Modena MG, Jaffe AS. Vascular surgery patients : perioperative and long term risk according to the ACC/AHA Guidelines, the additive role of post-operative troponin elevation. Eur Heart J. 2005 ; 26 (22) : 2448 - 2456

${ }^{26}$ Ali ZA, Callaghan CJ, Ali AA, Sheikh AY, Akhtar A, Pavlovic A, Nourai SA, Dukta DP, Gaunt ME. Perioperative myocardial injury after elective open abdominal aortic aneurysm repair predicts outcome. Eur J Vasc Endovasc Surg. 2008 Apr ; 35(4) : $413-9$

27 Kim JL, Martinez EA, Faraday N, Dorman T, Fleisher LA, Perler BA, Williams GM, Chan D, Pronovost PJ. Cardiac Troponin I predicts short term mortality in vascular surgery patients. Circulation 2002 ; 106 : 2366 - 2371

${ }^{28}$ Marston N, Brenes J, Garcia S, Kuskowski M, Adabag S, Santilli S, McFalls EO. Peak postoperative troponin levels outperform preoperative predictors of long term mortality after vascular surgery troponins and postoperative outcomes. J Crit Care. 2012 Feb ; 27(1) : $66-72$

${ }^{29}$ Landesberg G, Shatz V, Akopnik I, Wolf YG, Mayer M, Berlantzky Y, Weissman C, Mosseri M. Association of cardiac troponin, CK-MB, and postoperative myocardial ischemia with long-term survival after major vascular surgery. J Am Coll Cardiol. $2003 ; 42(9): 1547-1554$

${ }^{30}$ Lavi R, Lavi S, Daghini E, Lerman LO. New frontiers in the evaluation of cardiac patients for non cardiac surgery. Anaesthesiology 2007 ; 107 : 1018 - 1028

${ }^{31}$ Biccard BM, Roseth RN. Utility of clinical risk predictors for preoperative cardiovascular risk prediction. Br J Anaesth ; 107 (2) : 133 - 143

${ }^{32}$ Lee TH, Marcantonio ER, Mangione CM, Thomas EJ, Polaczyk CA, Cook EF, Sugarbaker DJ, Donaldson MC, Poss R, Ho KKL, Ludwig LE, Pedan A, Golman L. Derivation and prospective validation of a simple index for prediction of cardiac of major non cardiac surgery. Circulation $1999 ; 100$ : 1043 - 1049 
${ }^{33}$ Piriou V. Surveillance post opératoire du coronarien. QFP SFAR. www.sfar.org (consulté le 10/06/2012)

${ }^{34}$ Badner NH, Knill RL, Brown JE, Novick TV, Gelb AW. Myocardial infarction after noncardiac surgery. Anesthesiology $1998 ; 88(3)$ : 572 - 578

${ }^{35}$ Edouard A, Cosson C. Le dosage de la troponine I : intérêt et limites en pratique médicale courante. Rev Med Interne. 2003 ; 24 (9) : 623 - 626

36 Jaffe AS, Ravkilde J, Roberts R, Naslund U, Apple FS, Galvani M, Katus H. It's time for a change to a troponin standard. Circulation $2000 ; 102: 1216-1220$

${ }^{37} \mathrm{Wu}$ AHB, Jaffe AS. The clinical need for high-sensitivity cardiac troponin assays for acute coronary syndromes and the role for serial testing. Am Heart J. 2008 feb ; 155 (2) : $208-214$

${ }^{38}$ Philip I, Provenchère S, Merceron S, Toussaint A, Berroeta C, Dehoux M. Intérêt des marqueurs biologiques de la souffrance myocardique en périopératoire. Le praticien en anesthésie réanimation 2009 june ; 13 (3) : 183 - 194

${ }^{39}$ Reichlin T, Hochholzer W, Bassetti S, Steuer S, Stelzig C, Hartwiger S, Biedert S, Schaub N, Buerge C, Potocki M, Noveanu M, Breidthardt T, Twerenbold R, Winkler $\mathrm{K}$, Bingsser R, Mueller C. Early diagnosis of myocardial infarction with sensitive cardiac troponin assays. N Engl J Med. 2009 aug ; 361 : 858 - 867

${ }^{40}$ Lefevre G. Troponine T hypersensible : rupture ou continuité ? Cardiologie pratique, l'hebdomadaire du cardiologue 2009 jun ; 932 (2) ; 1

${ }^{41} 26^{\text {ème }}$ salon consensus cardio \& pratique. La troponine hypersensible, un nouvel outil diagnostique et pronostique. Consensus online 2010 (consulté le 24/08/2012)

${ }^{42}$ Giannitsis E, Bercker M, Kurz K, Hess G, Zdunek D, Katus H. High-sensitivity cardiac troponin $\mathrm{T}$ for early prediction of evolving non-ST-segment elevation myocardial infarction in patients with suspected acute coronary syndrome and negative troponin results on admission. Clin Chem. 2010 apr ; 56 : 4642 - 4650 
${ }^{43}$ Asseman P, Ennezat PV, Auffray JL, Bauchart JJ. Troponine haute sensibilité : quel mode d'emploi ? Cardiologie pratique, l'hebdomadaire du cardiologue 2009 jun ; $932(2) ; 1-4$

44 Dawood MM, Gutpa DK, Southern J, Walia A, Atkinson JB, Eagle KA. Pathology of fatal perioperative myocardial infarction: implications regarding pathophysiology and prevention. Int J Cardiol. 1996 Nov 15 ; 57(1) : 37 - 44

${ }^{45}$ Cohen MC, Aretz TH. Histological analysis of coronary artery lesions in fatal post operative myocardial infarction. Cardiovasc Pathol $1999 ; 8: 133$ - 9

${ }^{46}$ Paul SD, Eagle KA, Kuntz KM, Young JR, Hertzer NR. Concordance of preoperative clinical risk with angiographic severity of coronary artery disease in patients undergoing vascular surgery. Circulation 1996 ; 94 : 1561-6

${ }^{47}$ Schuetz GM, Zacharopoulou NM, Schlattmann P, Dewey M. Meta analysis : non invasive coronary angiography using computed tomography versus magnetic resonance imaging. Ann Intern Med. 2010 : 152167 - 177

${ }^{48}$ Philippe F. Quelle méthode pour prédire le risque coronaire en 2003 ? Aspects réglementaires et pratiques de l'utilisation de la coronarographie, de I'IRM et de l'angioscanner. Ann Cardiol angeiol (Paris). 2004 ; 53 : 79 - 90

${ }^{49}$ Bellenger NG, Burgess MI, Ray SG, Lahiri A, Coats AJS, Cleland JGF, Pennell DJ. CHRISTMAS study steering committee and investigators. Comparison of left ventricular ejection fraction and volumes in heart failure by echocardiography, radionuclide ventriculography and cardiovascular magnetic resonance. Eur Heart J. $2000 ; 21: 1387-1396$

50 Jaarsma C, Leiner T, Bekkers S, Crijns H, Wildberger J, Nagel E, Nelemans P, Schalla S. Diagnostic performance of PET, SPECT and IRMC perfusion imaging for the detection of significant coronary artery disease - a meta-analysis. J Cardiovasc Magn Reson. 2011 ; 13 (Suppl 1) : P75

${ }^{51}$ Schwitter J, Wacker CM, Van Rossum AC, Lombardi M, Al-Saadi N, Ahlstrom H, Dill T, Larsson HBW, Flamm SD, Marquardt M, Johansson L. MR-IMPACT : 
comparison of perfusion - cardiac magnetic resonance with single photon emission computed tomography for the detection of coronary artery disease in a multicentre, multivendor, randomized trial. Eur Heart J. 2008 ; 29 : 480 - 489

${ }^{52}$ Bandettini WP, Arai AE. Advances in clinical applications of cardiovascular magnetic resonance imaging. Heart 2008 Nov ; 94(11) : 1485 - 1495

${ }^{53}$ Watkins S, Oldroyd KG, Frohwein S. Magnetic resonance myocardial perfusion imaging : a new era in the detection of reversible myocardial ischaemia. Heart 2007 ; $93: 7-10$

${ }^{54}$ Germain P, Koenig A, Rochoux G, Jahn C, Coulbois PM, Frenzel B, Jeung MY. Principes physiques. [en ligne]. In : Recueil d'IRM cardiaque. Principes, semiology \& collection d'observations cliniques. Disponible sur http://irmcardiaque.com/index.php?title=Principes physiques (page consultée le 20/08/2012)

${ }^{55}$ Cheong B, Muthupillai R. Nephrogenic systemic fibrosis : a review. Tex Heart Inst J. $2010 ; 37(5): 508-515$

${ }^{56}$ Bandettini WP, Arai AE. Advances in clinical applications of cardiovascular magnetic resonance imaging. Heart 2008 November ; 94(11) : 1485 - 1495

${ }^{57}$ Plein S, Kozerke S, Suerder D, Luescher TF, Greenwood JP, Boesiger P, Schwitter J. High spatial resolution myocardial perfusion cardiac magnetic resonance for the detection of coronary artery disease. Eur Heart J. $2008 ; 29: 2148-2155$

${ }^{58}$ Schwitter J. Myocardial perfusion. J Magn Reson Imaging. 2006 Nov ; 24(5) : 953 $-63$

${ }^{59}$ Yan AT, Gibson CM, Larose E, Anavekar NS, Tsang S, Solomon SD, Reynolds G, Kwong RY. Characterization of microvascular dysfunction after acute myocardial infarction by cardiovascular magnetic resonance first pass perfusion and late gadolinium enhancement imaging. J Cardiovasc Magn Reson. 2006 ; 8(6) : 831 - 7

${ }^{60}$ Cerqueira MD, Weissman NJ, Dilsizian V, et al. Standardized myocardial segmentation and nomenclature for tomographic imaging of the heart: a statement 
for healthcare professionals from the cardiac imaging committee of the council on clinical cardiology of the American Heart Association. Circulation 2002 ; 105 : 539 542

61 Schuijf JD, Shaw LJ, Wijns W, Lamb HJ, Poldermans D, de Roos A, Vand der Wall EE, Bax JJ. Cardiac imaging in coronary artery disease : differing modalities. Heart $2005 ; 91: 1110-1117$

62 Yan AT, Gibson CM, Larose E, Anavekar NS, Tsang S, Solomon SD, Reynolds G, Kwong RY. Characterization of microvascular dysfunction after acute myocardial infarction by cardiovascular magnetic resonance first pass perfusion and late gadolinium enhancement imaging. J Cardiovasc Magn Reson. 2006 ; 8(6) : 831 - 7

${ }^{63}$ Mahrholdt H, Klem I, Sechtem U. Cardiovascular MRI for detection of myocardial viability and ischaemia. Heart $2007 ; 93: 122-129$

${ }^{64}$ Manrique A, Gerbaud E, Derumeaux G, Cribier A, Bertrand D, Lebon A, Dacher JN. Cardiac magnetic resonance demonstrates myocardial oedema in remote tissue early after reperfused myocardial infarction. Arch Cardiovasc Dis. 2009 Aug-Sep ; $102(8-9): 633-9$

${ }^{65}$ Barbou F, Lahutte M, Schiano P, Monsegu J. Detection of induced myocardial ischemia during stress cardiovascular magnetic resonance. Ann Cardiol Angeiol (Paris) 2011 Feb ; 60(1) : 42 - 7. Epub 2010 Dec 21. Review. French. PMID 20658242

${ }^{66}$ American College of Cardiology Foundation Task Force on Expert Consensus Documents, Hundley WG, Bluemke DA, Finn JP, Flamm SD, Fogel MA, Friedrich MG, Ho VB, Jerosch-Jerold M, Kramer CM, Manning WJ, Patel M, Pohost GM, Stillman AE, White RD, Woodard PK. ACCF/ACR/NASCI/SIRMC 2010 expert consensus document on cardiovascular magnetic resonance : a report of the American College of Cardiology Foundation Task Force on Expert Consensus Documents. J Am Coll Cardiol. 2010 Jun ; 55(23) : 2614 - 62 
${ }^{67}$ Larose E, Rodés-Cabau J, Delarochelliere R, Barbeau G, Noel B, Bertrand O. Cardiovascular magnetic resonance for the clinical cardiologist. Can J Cardiol. 2007 Oct ; 23 Suppl B : 84B - 88B

${ }^{68}$ Ibrahim T, Hubertus P, Bülow P, Hackl T, Hörnke M, Nekolla SG, Breuer M, Schömig A, Schwaiger M. Diagnostic value of contrast-enhanced magnetic resonance imaging and single-photon emission computed tomography for detection of myocardial necrosis early after acute myocardial infarction. J Am Coll Cardiol. $2007 ; 49(2): 208-216$

${ }^{69}$ Gibbons RJ, Valeti US, Araoz PA, Jaffe AS. The quantification of infarct size. J Am Coll Cardiol. 2004 Oct ; 44(8) : $1533-42$

${ }^{70}$ Référentiel de pratiques professionnelles. Transfusion en anesthésie réanimation. Juin 2005. http://www.has-sante.fr/portail/upload/docs/application/pdf/200912/antibio perioperatoire ref.pdf in [www.has-sante.fr] (consulté le 22/09/2012)

${ }^{71}$ Muller M, Duperret S. 2007. Territoires coronariens, http://www.echorea.org/images/territoires coronaires.jpg, in [www.echorea.org] (consulté le 22/09/2012)

${ }^{72}$ Lebard C. Détection de la nécrose myocardique post opératoire en chirurgie vasculaire - comparaison de la troponine sensible à la troponine standard [Thèse]. Anesthésie réanimation chirurgicale : Paris ; 2010. 68 p.

${ }^{73}$ Hollander JE. Highly sensitive troponins the answer or just more questions ? J Am Coll Cardiol. $2009 ; 54: 1173-5$

${ }^{74}$ Poldermans D, Boersma E, Bax JJ, Kliffen M, van Urk H, van de Ven L, Roelandt JRTC, Thomson IR. Correlation of location of acute myocardial infarct after non cardiac vascular surgery with pre operative dobutamine echocardiographic findings. Am J Cardiol. 2001 Dec 15 ; 88(12) : 1413 - 4, A6

${ }^{75}$ Wu KC, Kim RJ, Bluemke DA, Rochitte CE, Zerhouni EA, Becker LC, Lima JAC. Quantification and time course of microvascular obstruction by contrast-enhanced 
echocardiography and magnetic resonance imaging following acute myocardial infarction and reperfusion. J Am Coll Cardiol. 1998 ; 32(6) : 1756 - 1764

${ }^{76}$ Raman SV, Simonetti OP, Winner MW, Dickerson JA, He X, Mazzaferri EL, Ambrosio G. Cardiac magnetic resonance with edema imaging identifies myocardium at risk and predicts worse outcome in patients with non ST segment elevation acute coronary syndrome. J Am Coll Cardiol 2010 June ; 55(22) : 2480 - 8

${ }^{77}$ Hundley WG, Bluemke D, Bogaert JG, Friedrich MG, Higgins CB, Lawson MA, McConnell MV, Raman SV, van Rossum AC, Flamm S, Kramer CM, Nagel E, Neubauer S. Society for cardiovascular magnetic resonance guidelines for reporting cardiovascular magnetic resonance examinations. J Cardiovasc Magn Reson. 2009 ; $11: 5$

${ }^{78}$ Wagner A, Mahrholdt H, Holly TA, Elliott MD, Regenfus M, Parker M, Klocke FJ, Bonow RO, Kim RJ, Judd RM. Contrast-enhanced MRI and routine single photon emission computed tomography (SPECT) perfusion imaging for detection of subendocardial myocardial infarcts: an imaging study. The Lancet. 2003 Feb ; 361 : $374-79$

${ }^{79}$ Kim RJ, Wu E, Rafael A, Chen EL, Parker MA, Simonetti O, Klocke FJ, Bonow RO, Judd RM. The use of contrast-enhanced magnetic resonance imaging to identify reversible myocardial dysfunction. N Engl J Med $2000 ; 343: 1445$ - 53 


\section{ANNEXE 1 \\ REVISED CARDIAC RISK INDEX ET CAPACITE FONCTIONNELLE}

REVISED CARDIAC RISK INDEX

(Circulation 1999; 100:1043-1049)

Each risk factor is assigned one point.

1. High-risk surgical procedures

- Intraperitoneal

- Intrathoracic

- Suprainguinal vascular

2. History of ischemic heart disease

- History of myocardial infarction

- History of positive exercise test

- Current complain of chest pain considered secondary to myocardial ischemia

- Use of nitrate therapy

- ECG with pathological $Q$ waves

3. History of congestive heart failure

- History of congestive heart failure

- Pulmonary edema

- Paroxysmal nocturnal dyspnea

- Bilateral rales or $\$ 3$ gallop

- Chest radiograph showing pulmonary vascular redistribution

4. History of cerebrovascular disease

- History of transient ischemic attack or stroke

5. Preoperative treatment with insulin

6. Preoperative serum creatinine $>2.0 \mathrm{mg} / \mathrm{dL}$

\section{RISK OF MAJOR CARDIAC EVENT}

\begin{tabular}{cccc} 
Points & Class & Risk \\
\cline { 2 - 3 } & I & $0.4 \%$ \\
1 & II & $0.9 \%$ \\
2 & III & $6.6 \%$ \\
3 or more & IV & & $11 \%$
\end{tabular}

"Major cardiac event" includes myocardial infarction, pulmonary edema, ventricular fibrillation, primary cardiac arrest, and complete heart block 
$1 \mathrm{MET}=3,5 \mathrm{ml} \mathrm{O} / \mathrm{kg} / \mathrm{min}$.

1-4 MET

- Activité de ménage

- Marche sur chemin plat, $500 \mathrm{~m}$, $\max 4 \mathrm{~km} / \mathrm{h}$

5-9 MET

- Marche en montant

- $\geq 2$ étages d'escaliers

- Travail lourd en position assise

- Sports modérés (natation, trecking, golf)

$>9$ MET

- Sports intensifs (tennis, cyclisme, course, escalade de rocher)

- Travail physique intense (travailleur de construction, abattage de bois)

Figure 25 : capacité fonctionnelle 


\section{ANNEXE 2 \\ IMAGES DE REHAUSSEMENT TARDIF DE LESIONS MYOCARDIQUES}

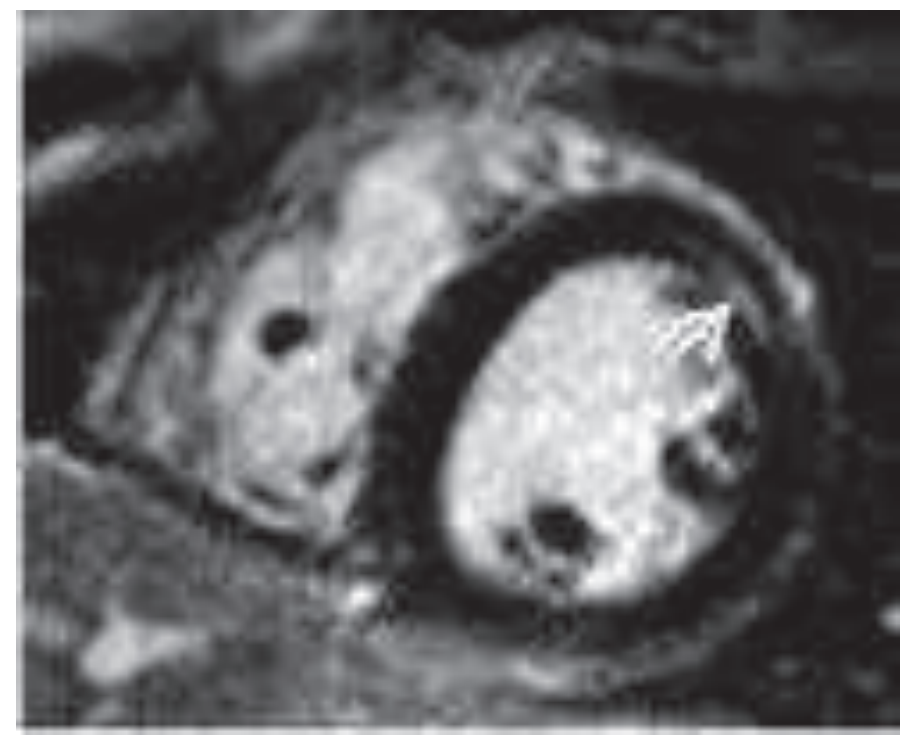

Figure 26 : infarctus myocardique antérieur aigu vu en IRM cardiaque sur les séquences de rehaussement tardif

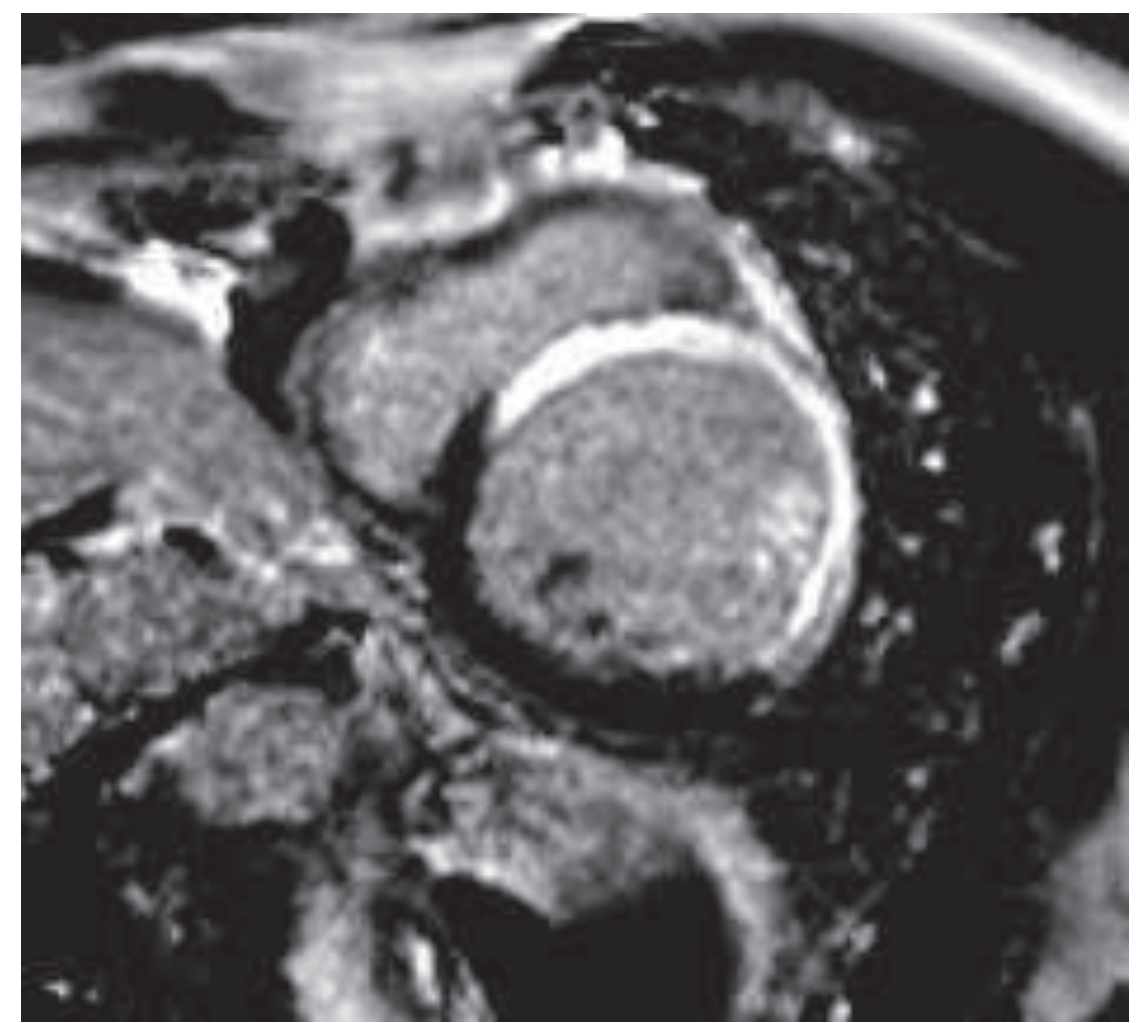

Figure 27 : infarctus myocardique antéro-septal transmural vu en IRM cardiaque sur les séquences de rehaussement tardif 


\section{ANNEXE 3}

\section{EXEMPLE DE PROTOCOLE DE PRISE EN CHARGE DE SYNDROMES CORONARIENS AIGUS (SCA) POST OPERATOIRES MIS EN PLACE AUX HOSPICES CIVILS DE LYON}

\begin{tabular}{|c|c|c|c|}
\hline RAPPIL & HAUT RISQUE & $\begin{array}{c}\text { RISQUE } \\
\text { INTERMEDIAIRE }\end{array}$ & BAS RISQUE \\
\hline $\begin{array}{l}\frac{\text { RISQUE VITAL. IMMEDIAT }}{\text { (quelle que soit la valeur }} \\
\text { troponine }+t+) \\
\text {-Choe cardiogénique } \\
=\text { hypoTA + VG altéré en écho } \\
\text {-etiou Sus décalage ST }\end{array}$ & $\begin{array}{l}\text { Signes eliniques } \\
\text { d'insuffisance } \\
\text { cardiaque } \\
\text { ET dysfonction } \\
\text { VG à l'écho }\end{array}$ & $\begin{array}{l}\text { 1) Signes cliniques } \\
\text { d'insuffisance cardiaque } \\
\text { mais fonction VG } \\
\text { systolique normale à I'echo } \\
\text { 2) Fonction systolique VG } \\
\text { anormale à Y'écho mais pas }\end{array}$ & $\begin{array}{l}3 \text { conditions } \\
\text { - pas de } \\
\text { symptôme } \\
\text { - Echo normale } \\
\text { - Troponine <3 }\end{array}$ \\
\hline $\begin{array}{l}\text { TRAXSFERT direct en } \\
\text { salle de coronarographie }\end{array}$ & $\begin{array}{l}\text { Douleur } \\
\text { thoracique } \\
\text { typiquement }\end{array}$ & $\begin{array}{l}\text { rapport ECG de tétérence } \\
\text { sans autre anomalie clinique } \\
\text { ou écho }\end{array}$ & \\
\hline $\begin{array}{l}\text {-Appel SAMU : tel : .... } \\
\text {-Urgence coronaire: tel : .... }\end{array}$ & $\begin{array}{l}\text { persistante ET } \\
\text { nouveau sous- } \\
\text { décalage de ST }\end{array}$ & & \\
\hline $\begin{array}{c}\text { Si assistance circulatoire } \\
\text { envisapée (BCPLA ou } \\
\text { ECMO) } \\
\text { tel : .... }\end{array}$ & & & \\
\hline
\end{tabular}

Figure 29 : exemple de prise en charge de SCA post opératoire mis en place aux hospices civils de Lyon : définition du risque d'après Piriou et al. $2010^{5}$.

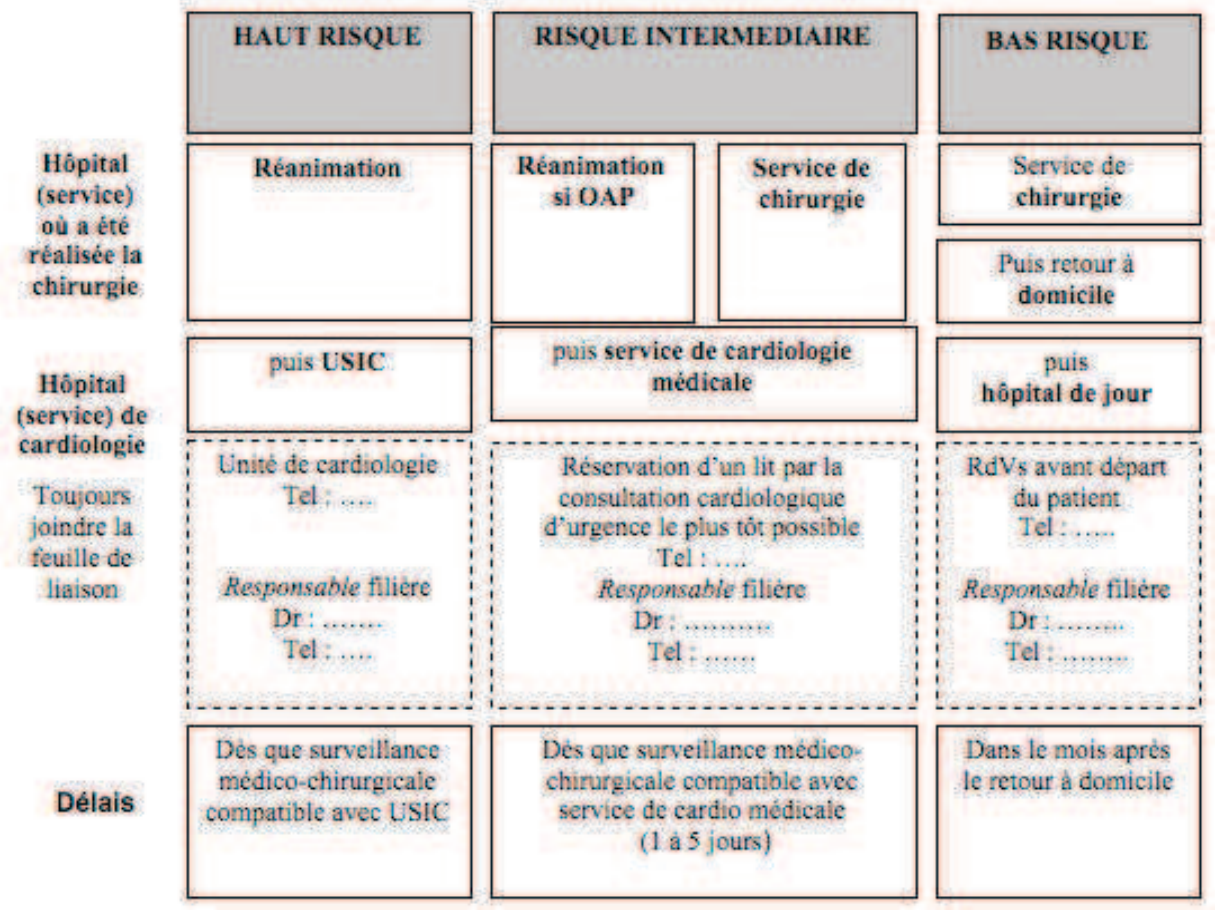

Figure 30 : exemple de prise en charge de SCA post opératoire mis en place aux hospices civils de Lyon : orientation selon le risque d'après Piriou et al. $2010^{5}$. 

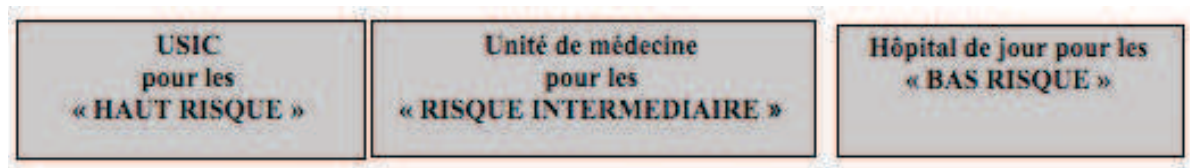

-Echocardiographie
-Coronarographie rapide
$(<12 \mathrm{~h})$ et revascularisation
médicale ou chirurgicale
-Pas de stent actif
-Stent, anti-gpllbIlla,
anticoagulation selon les
indications de la feuille de
liaison
-BCPIA si nécessaire

\begin{tabular}{|c|l|}
\hline $\begin{array}{c}\text { RETOUR CENTRE } \\
\text { HOSPITALIER } \\
\text { D'ORIGINE }\end{array}$ & $\begin{array}{l}\text { - La stratégie de revascularisation } \\
\text { tient compte des indications de la } \\
\text { feuille de liaison }\end{array}$ \\
$\begin{array}{c}\text { Réanimation dés que l'état } \\
\text { cardiologique est stabilisé - } \\
\text { retour avec BCPIA possible }\end{array}$ & $\begin{array}{l}\text { Organisation du suivi cardiologique } \\
\text { éventuel }\end{array}$ \\
\hline
\end{tabular}

\begin{tabular}{|l|}
\hline - Evaluation des facteurs \\
de risque \\
- Echocardiographie \\
- Test d'ischémie non \\
invasif (écho dobu ou \\
épreuve d'effort) \\
- Organisation du suivi \\
cardiologioue éventuel \\
\hline
\end{tabular}

-Autre artère : revascularisation ou test d'ischémie selon symptòmes actuels et avant chirurgie, fonction $\mathrm{VG}$, localisation, nombre et complexité des tésions coronaires

- Coronarographie $<24 \mathrm{~h}$

- Si coronaires saines : IRM

- Si lésion aiguë : angioplastie

- Si atteinte coronaire chronique

-IVA prox ou moyenne:

NA prox ou moyc

SORTIE vers le domicile ou

convalescence selon ce qui a èté

organisé dans le centre ou la

chururgie a èté réalisée

Figure 31 : exemple de prise en charge de SCA post opératoire mis en place aux hospices civils de Lyon : gestion du patient en cardiologie d'après Piriou et al. $2010^{5}$. ECMO : système d'oxygénation extra corporelle à membrane, USIC : unité de soins intensifs de cardiologie, BPCIA : ballon de contre pulsion intra aortique 


\section{Résumé}

En chirurgie non cardiaque, une élévation de la troponine est associée à une augmentation de la morbi-mortalité d'origine cardio-vasculaire à court et à long termes. La mortalité d'origine cardiaque est proportionnelle au pic du biomarqueur.

L'IRM cardiaque permet l'exploration du lit micro-vasculaire du myocarde et la caractérisation des lésions.

\section{Matériel et méthodes}

Tous les patients opérés entre le 31 mars 2010 et le 31 décembre 2011, ayant présenté une élévation de troponine sur 2 dosages consécutifs supérieurs au $99^{\text {ème }}$ percentile, ont été étudiés. II a été réalisé une IRM cardiaque avec injection de gadolinium pour étude du rehaussement tardif permettant l'étude micro-circulatoire chez tous les patients éligibles.

\section{Résultats}

Sur les 249 patients ayant présenté une élévation de troponine sur la période de l'étude, seuls 24 ont pu bénéficier d'une IRM cardiaque. Les élévations de troponine survenaient majoritairement $(79 \%)$ dans les 48 premières heures.

L'IRM était réalisée dans un délai médian de 81,5 jours. Quatorze fois, I'IRM a identifié un dommage myocardique correspondant à une altération de la microcirculation, 8 fois transmurale et 6 fois entre 25 et $75 \%$ de l'épaisseur du myocarde. Ces lésions sont indépendantes des zones de sténose coronaire connues en pré opératoire.

La mortalité à 1 an est de $41,6 \%$ dans le groupe de patients étudiés.

\section{Discussion}

Le délai de réalisation de I'IRM de 81 jours peut rendre compte de la négativité de l'IRM.

Le coût de l'examen et la lourdeur de sa réalisation ne peuvent justifier sa réalisation en dehors d'une étude clinique.

\section{Conclusion}

Les élévations de troponine en post opératoire de chirurgie vasculaire et thoracique sont associées à des dommages myocardiques par altération de la microcirculation visibles en IRM cardiaque.

L'étude de la micro-circulation par IRM authentifie la réalité des dommages myocardiques à distance d'un acte chirurgical.

La mortalité élevée de ces patients implique la mise en œuvre d'un traitement médical précoce adapté.

Mots clés : IRM myocardique, dommage myocardique post opératoire, élévation de troponine post opératoire 